\title{
Hydrogeology of Unconsolidated Deposits in Chenango County, New York
}

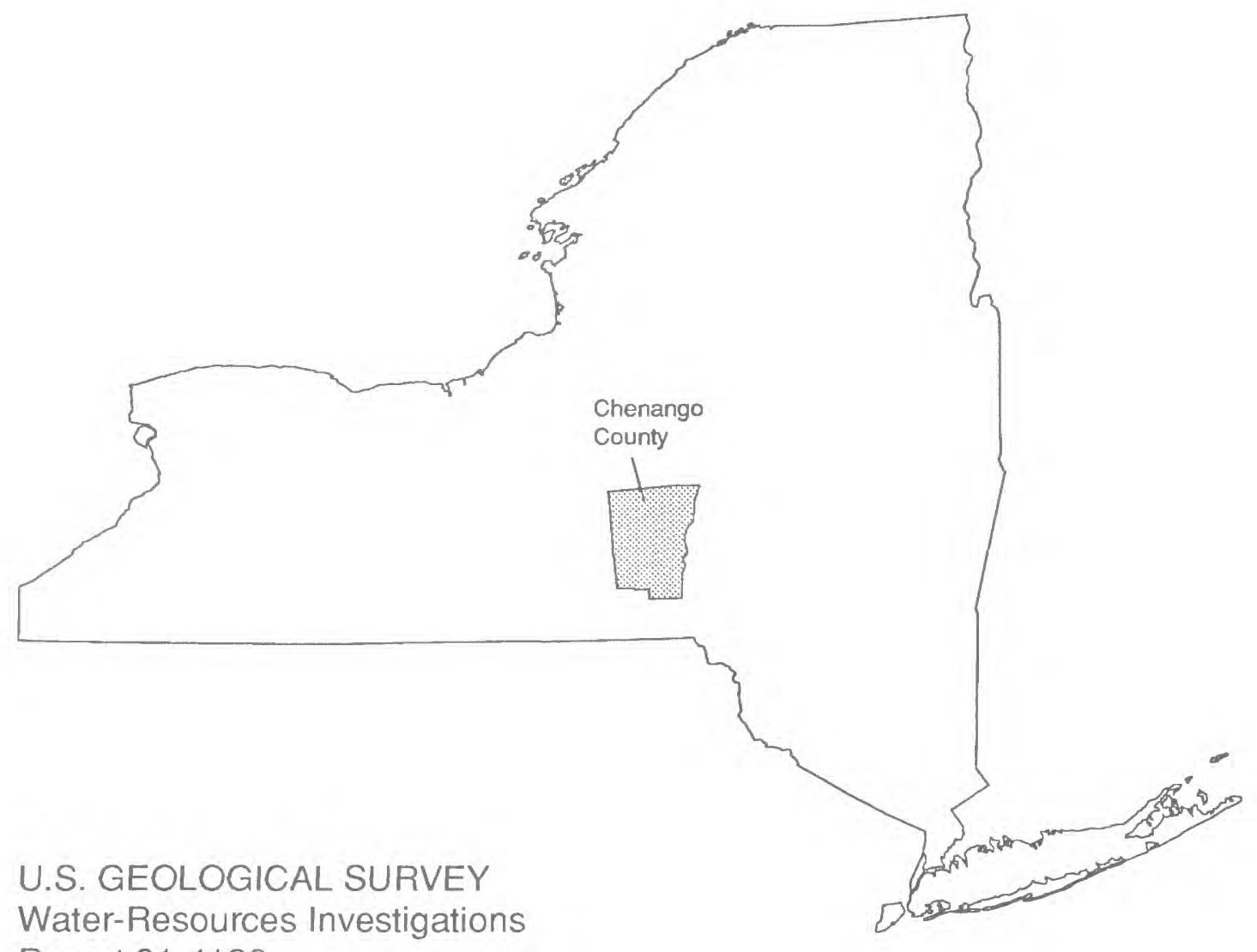

Report 91-4138

Prepared in cooperation with CHENANGO COUNTY 
HYDROGEOLOGY OF UNCONSOLIDATED DEPOSITS

IN CHENANGO COUNTY, NEW YORK

By Wendy S. McPherson

\section{U.S. GEOLOGICAL SURVEY}

Water-Resources Investigations Report 91-4138

Prepared in cooperation with the CHENANGO COUNTY

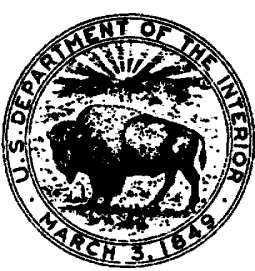

Ithaca, New York

1993 


\section{U.S. DEPARTMENT OF THE INTERIOR}

BRUCE BABBITT, Secretary

U.S. GEOLOGICAL SURVEY

Dallas L. Peck, Director

For additional information write to:

Subdistrict Chief

U.S. Geological Survey

903 Hanshaw Road

Ithaca, NY 14850-1573
Copies of this report can be purchased from:

U.S. Geological Survey Books and Open-File Reports Section Federal Center

Box 25425

Denver, CO 80225 


\section{CONTENTS}

\begin{tabular}{|c|}
\hline Abstract ....................... \\
\hline Introduction ... \\
\hline 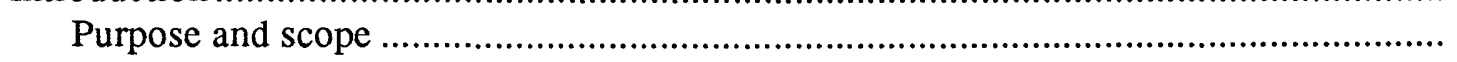 \\
\hline Description of study area.......... \\
\hline 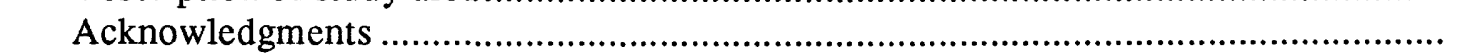 \\
\hline 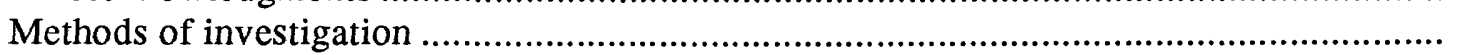 \\
\hline 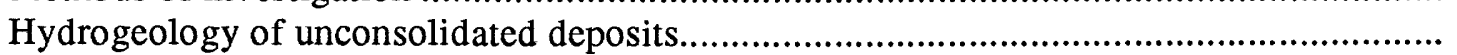 \\
\hline Types of deposits \\
\hline Stratified drift \\
\hline Till \\
\hline Moraine \\
\hline Alluvium and colluvium \\
\hline Thickness \\
\hline Potential well yields from valley-fill aquifers \\
\hline Immary \\
\hline lected references \\
\hline
\end{tabular}

\section{ILLUSTRATIONS}

Plates 1-3. Maps of Chenango County (in pocket) showing:

1. Location of wells and test holes.
A. Southern part.
B. Northern part.

2. Thickness of unconsolidated deposits.
A. Southern part.
B. Northern part.

3. Potential well yields from valley-fill aquifers.

A. Southern part.

B. Northern part.

Figure 1. Map showing location of Chenango County and physiographic provinces of New York

2. Block diagram showing a till-shadow hill within a typical upland tributary valley in Chenango County

\section{TABLE}

Table 1. Records of wells and test holes 
CONVERSION FACTORS AND VERTICAL DATUM

Multiply

inch (in.)

foot (ft)

mile (mi)

square mile $\left(\mathrm{mi}^{2}\right)$

gallon per minute (gal/min)
By

To obtain

25.4

0.3048

1.609

2.59

0.06308 millimeter

meter

kilometer

square kilometer

liter per second

Sea level: In this report, "sea level" refers to the National Geodetic Vertical Datum of 1929-a geodetic datum derived from a general adjustment of the first-order level nets of the United States and Canada, formerly called Sea Level Datum of 1929. 


\title{
HYDROGEOLOGY OF UNCONSOLIDATED DEPOSITS IN CHENANGO COUNTY, NEW YORK
}

\author{
By Wendy S. McPherson
}

\begin{abstract}
Chenango County uses ground water from unconsolidated valley-fill aquifers and from bedrock in the uplands for public water supply. Water-resources managers and planners need information on the location, thickness, and potential well yield of unconsolidated deposits throughout the county to formulate plans to protect and manage the ground-water resources. Data from 519 well sites were obtained from well drillers and several State agencies in 1989 and 1990 and combined with data from previous studies to compile a well-inventory table and maps depicting well locations, thickness of unconsolidated deposits, and potential well yields from valley-fill aquifers.

Thickness of unconsolidated deposits on hilltops and in areas of steep terrain is generally less than $5 \mathrm{ft}$ (feet); on north-facing hillsides it is generally less than $50 \mathrm{ft}$, whereas on south-facing hillsides and kame terraces, it typically ranges from 50 to $100 \mathrm{ft}$. Thickness of unconsolidated deposits in major stream valleys generally exceeds $100 \mathrm{ft}$ and exceeds $200 \mathrm{ft}$ locally.

The most productive aquifers consist of coarse valley-fill sand and gravel deposits. Wells completed in aquifers that occupy minor stream valleys, such as the Genegantslet Creek Valley, generally yield less than $100 \mathrm{gal} / \mathrm{min}$ (gallons per minute). Potential well yields from deposits in major stream valleys, such as the Chenango River Valley, range from 100 to $3,500 \mathrm{gal} / \mathrm{min}$. In areas where the saturated thickness exceeds $40 \mathrm{ft}$ and the drainage basin exceeds 200 square miles, the potential yield of some wells can exceed $3,500 \mathrm{gal} / \mathrm{min}$.
\end{abstract}

\section{INTRODUCTION}

Chenango County uses ground water from unconsolidated valley-fill aquifers and from bedrock in the uplands for public water supply. To formulate plans to protect and manage the groundwater resources, water-resource managers and planners need information on the thickness of unconsolidated deposits because the thickness of these materials largely determines the degree to which ground water is protected from surface contaminants; they also need to know the location and potential yield of wells completed in such aquifers. Because only minimal information on the unconsolidated deposits of Chenango County is available, a hydrogeologic data base and maps showing the thickness of unconsolidated deposits and the extent and potential well yields in valleyfill aquifers are needed as a basis for ground-water management plans.
In 1989, the U.S. Geological Survey, in cooperation with Chenango County, began a 2-year study to provide hydrogeologic information, obtained from well drillers and State agencies, to county planners.

\section{Purpose and Scope}

This report describes hydrogeologic characteristics of the unconsolidated deposits in Chenango County as inferred from well and testhole data. It includes an inventory of wells and test holes and a series of maps, at 1:48,000 scale, that show well locations (pl. 1A, 1B), thickness of unconsolidated deposits (P1. 2A, 2B), and potential well yields in valley-fill aquifers (pl. 3A, 3B). 


\section{Description of Study Area}

Chenango County is in the glaciated Appalachian Plateau physiographic province of southcentral New York State (fig. 1). The region encompasses $908 \mathrm{mi}^{2}$ (Crandall, 1985, p. 1) and was glaciated and modified several times during the Pleistocene Epoch. The last glaciation, which occurred during the Wisconsinan Stage of the Pleistocene Epoch (about 12,000 years ago), modified the landscape to its present form.

Elevations range from 880 to $1,960 \mathrm{ft}$ above sea level (Crandall, 1985, p. 2), and local relief may reach $600 \mathrm{ft}$ from the valley floor in major stream valleys. The average temperature for winter is $22^{\circ} \mathrm{F}$ and for summer is $65^{\circ} \mathrm{F}$; total annual precipitation averages 40 in. (Crandall, 1985 , p. 2). More than half the county is classified as forest; the remainder is agricultural, except for a small percentage that is classified as commercial, industrial, or residential (Crandall, 1985, p. 1).

Water supply in Chenango County is derived from surface-water reservoirs and ground water pumped from unconsolidated deposits in the valleys and bedrock in the uplands. Yields of wells that tap bedrock aquifers generally are smaller than those that tap the unconsolidated aquifers.

\section{Acknowledgments}

Thanks are extended to local well drillers, the Chenango County Department of Planning and Development; New York State Departments of Transportation, Environmental Conservation, and Health; and the New York State Geological Survey for providing well records and hydrogeologic information.

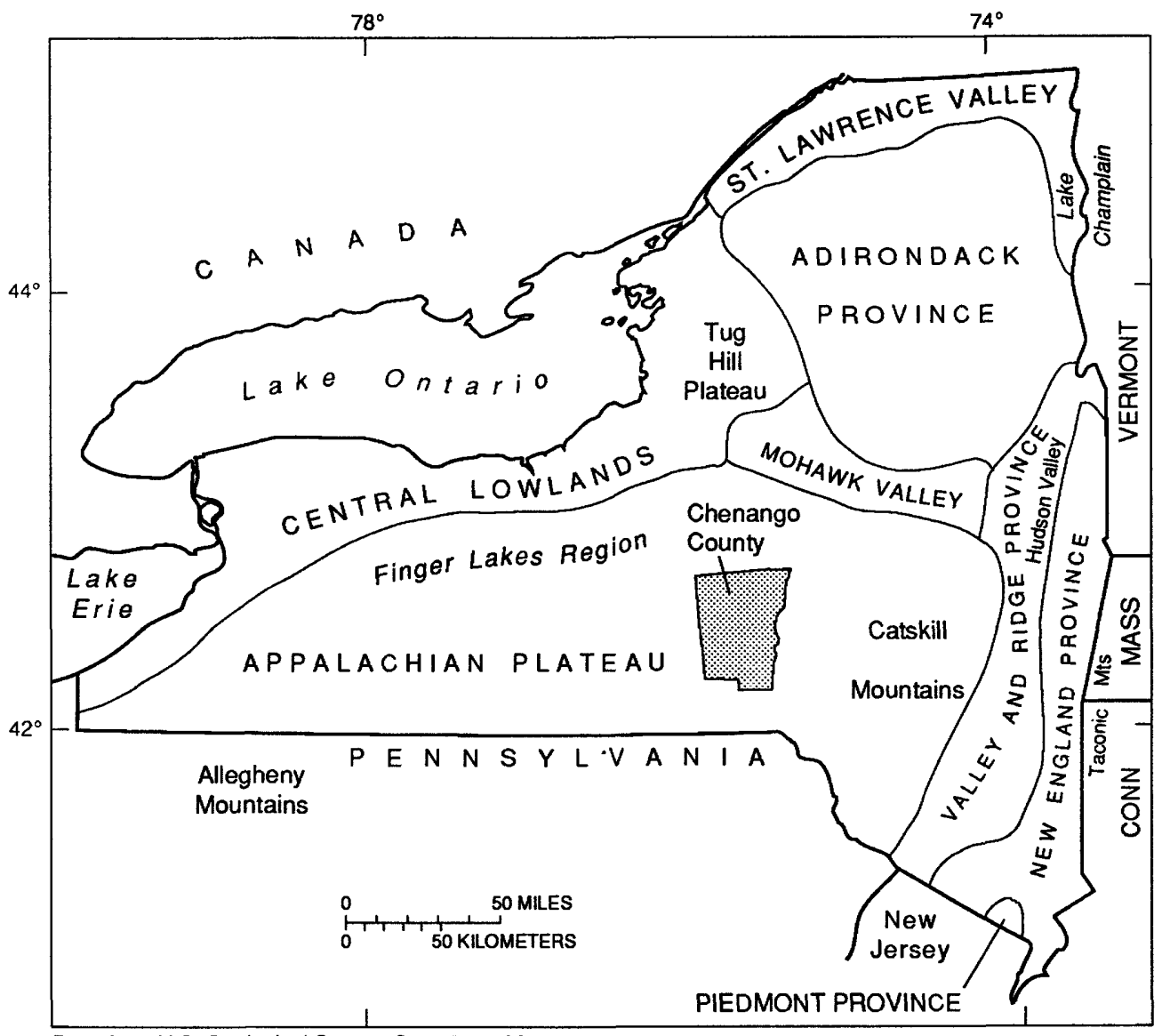

Base from U.S. Geological Survey, State Base Map Shaded relief, 1:500,000, 1956

Figure 1.--Location of Chenango County and physiographic provinces of New York. (Modified from Lyford and others, 1984, fig. 2; Lumia, 1991, fig. 1.) 


\section{METHODS OF INVESTIGATION}

Well and test-hole data were collected from local well drillers; the New York State Departments of Transportation, Environmental Conservation, and Health; and the New York State Geological Survey. The maps of Chenango County (pls. 1, 2, 3) have been arbitrarily divided into south (pl. A) and north (pl. B) sheets because the county is too large to depict conveniently at the selected scale $(1: 48,000)$. The well-location maps (pls. 1A, 1B) and record of wells and test holes (table 1, at end of report) were constructed from data from 840 sites, including 519 sites inventoried during this study and 321 from previously published reports (Randall, 1972; Reynolds and Brown, 1984).

Well and test-hole locations were derived from records of the owners of domestic wells and tax-parcel maps and from base maps or location coordinates given on geologic logs of observation wells and test holes. Well and test-hole locations were transferred to 7.5-minute topographic maps (1:24,000 scale) and digitized to derive the latitude and longitude in degrees, minutes, and seconds. Data from the wells and test holes were entered into the U.S. Geological Survey's Ground-Water Site Inventory computer data base, and wells were identified by numbers assigned according to a sequential numbering system arranged by county. Land-surface altitudes given for domestic wells were taken from topographic maps and are accurate to within $20 \mathrm{ft}$, but are affected by topography and the accuracy of the well location indicated in table 1. Yields of domestic wells were estimated by the drillers at the completion of the drilling.

Thickness of unconsolidated deposits (pls. 2A, 2B) was determined through analysis of soils maps, well and test-hole data, and surficial geology. Five thickness zones were identified from the sediment-thickness data; their boundaries are approximate and are not intended for use in sitespecific studies.

The Chenango County soil survey was done by the Soil Conservation Service (Crandall, 1985). Soil classifications were based on "steepness, length, and shape of the slopes; the general pattern of drainage; the kinds of crops and native plants growing on the soils; and the kinds of bedrock" (Crandall, 1985, p. 5). Patterns within these criteria form "landscape segments" that can be used to indicate soil types; soil types also are identified through physical characteristics, such as color, texture, water-filtration rate, and depth to bedrock (Crandall, 1985).

Seven types of soils that typically occupy areas where bedrock is less than $5 \mathrm{ft}$ below land surface were identified and delineated as zone 1 ; soils that typically have thickness greater than $5 \mathrm{ft}$ could not be used to infer depth to bedrock. Where the soil-type boundaries were not in agreement with the well data, the well data were considered the more accurate and were therefore used.

Subsequent delineation of zones 2 through 5 (described on p. 6) were based on casing data, data from geologic logs, and surficial geology. If data were insufficient to indicate whether a well was finished in bedrock or unconsolidated deposits, the following assumptions were made:

1. If the depth of the well was greater than the casing depth, the depth of casing was assumed equal to the thickness of unconsolidated deposits, and the well was assumed to terminate in bedrock.

2. If the depth of the well was equal to the depth of the casing, the well was assumed to penetrate only unconsolidated deposits, and the casing depth was taken to be a minimum thickness of unconsolidated deposits.

3. Measuring points were assumed to be at land surface unless otherwise stated.

All of Chenango County was glaciated, and the sediments that now fill the bedrock valleys were deposited by glaciofluvial meltwater, either as coarse-grained outwash from streams, or as fine-grained lacustrine sediments that filled temporary glacial lakes. Saturated, coarse-grained sand and gravel form valley-fill aquifers within these bedrock valleys. The water in these aquifers may be stored under confined or unconfined (water table) conditions. Boundaries of valley-fill aquifers shown in this report (pls. 3A, 3B) generally represent the contact between unconsolidated 
deposits and till or the bedrock valley wall. These aquifers were delineated from surficial geologic maps (Cadwell, 1972; Muller and Cadwell, 1986; and Cadwell and Dineen, 1987), geologic logs of wells and test holes, and soil maps (Crandall, 1985). In the absence of well data, the thickness of valley-fill deposits in major valleys, such as the Chenango River Valley, was estimated by projecting the slope of the adjacent bedrock valley walls downward, then subtracting the estimated altitude of the bedrock floor from that of the overlying valley floor. This method was also used in the narrow, steep-sided upland valleys (zones 1 or 2) to estimate sediment thickness. In areas not underlain by valley-fill aquifers, such as the uplands, ground water is obtained from bedrock. Yields of wells completed in bedrock vary widely and depend largely on the type of bedrock and its degree of fracturing.

Ground water in most of the valley-fill aquifers in Chenango County is under unconfined (water table) conditions. In unconfined aquifers, the water table represents the top of the saturated zone and is approximately at atmospheric pressure. The water table fluctuates in response to changes in natural recharge, discharge, and local ground-water pumpage. Potential well yields shown in this report for valley-fill aquifers were based on reported pumping rates of wells, yield estimates from drillers, saturated thickness of the aquifer, and a statistical analysis of well yield for wells in the Susquehanna River Basin (Hollyday, 1969). Ranges of potential well yields, given here, were estimated from saturated thickness and wellyield data. These ranges are conservative estimates and can be expected to be equalled or exceeded by 50 to 75 percent of the wells within each well-yield zone (pls. 3A, 3B). The ranges used are nearly the same as those used in a statistical analysis of well-yield data for the Susquehanna River Basin in New York (Hollyday, 1969, table 1, p. 48; and MacNish and Randall, 1982, table 1, p. 8) and are as follows:

\begin{tabular}{|c|c|c|}
\hline $\begin{array}{c}\text { Saturated } \\
\text { thickness } \\
\text { of aquifer } \\
(\mathrm{ft})\end{array}$ & $\begin{array}{l}\text { Potential } \\
\text { well yield } \\
\text { (gal/min) }\end{array}$ & $\begin{array}{l}\text { Well diameters } \\
\text { required to } \\
\text { obtain potential } \\
\text { well yields } \\
\text { (in.) }\end{array}$ \\
\hline $\begin{array}{l}\text { less than } 10 \\
10 \text { to } 40 \\
\text { greater than } 40\end{array}$ & $\begin{array}{l}\text { less than } 100 \\
100 \text { to } 1,500 \\
1,500 \text { to } 3,500\end{array}$ & $\begin{array}{l}6 \text { to } 8 \\
8 \text { to } 14 \\
14 \text { to } 20\end{array}$ \\
\hline
\end{tabular}

These zones of saturated thickness, as delineated by MacNish and Randall (1982, pl. 1B), were transferred to the potential-yield map and verified or updated with reported pumping rates of wells and yield estimates from drillers. The potential-yield range greater than or equal to 3,500 gal/min (zone 4) was assigned to regions having a saturated thickness greater than $40 \mathrm{ft}$, a drainage area greater than $200 \mathrm{mi}^{2}$, and an assumed hydraulic connection to major streams.

Saturated, well-sorted, coarse sand and gravel deposits that are overlain by relatively impermeable lacustrine sediment (fine-grained silt and clay) are called confined aquifers and are under pressure greater than atmospheric. The locations and estimated extent of confined aquifers within major valleys were taken from MacNish and Randall (1982, pl. 1B) and were inferred from the locations of artesian wells that were completed in stratified drift. Confined aquifers occur in many locations within the major valleys in Chenango County, but well data are generally insufficient to accurately delineate their thickness and extent.

\section{HYDROGEOLOGY OF UNCONSOLIDATED DEPOSITS}

Unconsolidated valley-fill aquifers occur in the major valleys that contain a river or large stream; these valleys are generally oriented northsouth in Chenango County. Many large residential and industrial areas obtain water from high-yielding wells that tap these productive valley-fill aquifers. Minor valleys that contain small streams are typically oriented east-west in Chenango County and generally lack high-yielding valley-fill aquifers.

\section{Types of Deposits}

Unconsolidated deposits in Chenango County are of several types, including glacially derived 
stratified drift, till and moraine, and recent sediment (alluvium and colluvium) deposited by postglacial streams.

\section{Stratified Drift}

Stratified drift (well sorted and layered) was deposited in the major valleys by glaciofluvial meltwater and includes outwash, ice-contact, and lacustrine deposits. Outwash deposits consist of well-sorted sand and gravel scoured from bedrock by ice and deposited near the center of the valleys by glacial meltwater. Ice-contact deposits, which include kames, kame deltas, kame terraces, and eskers, were formed during deglaciation between the ice and the valley walls or between the ice terminus and a temporary glacial lake. Ice-contact deposits generally consist of individual beds of poorly to well-sorted sand and gravel, cobbles, silt and clay. Lacustrine sand, silt, and clay were deposited in temporary lakes that formed during deglaciation where meltwater was impounded by ice or older deposits downvalley.

\section{Till}

Till is an unstratified, compact, unsorted mixture of cobbles, gravel, sand, silt, and clay that was deposited directly by and undemeath the advancing glacier. Till typically has low permeability because it was compacted by the weight of the glacier and was generally not sorted or reworked by subsequent meltwater streams. Till therefore does not transmit water readily and is considered to be poor aquifer material.
In the uplands, till directly overlies bedrock. As the glaciers advanced southward, they removed previously deposited till from the north-northeastfacing slopes and redeposited it on the southsouthwest-facing slopes. Till is therefore generally thinnest on the north-northeast-facing slopes and hilltops and thickest on the south-southwestfacing slopes, and these "till shadow" hills (fig. 2) are asymmetrical as a result (Coates, 1974, p. 224). In the Chenango River Basin, thick deposits of till generally mantle the bedrock walls and floors of minor valleys that are tributary to major southward-draining valleys (Cadwell, 1981). Till on the south-southwest-facing slopes increases in thickness downslope to a position about four-fifths the distance from the hilltop to the lowest point in the valley floor (the thalweg), then thins over the final fifth of this distance to the stream. The point at which till thickness is greatest is interpreted as the thalweg of the preglacial stream channel that occupied the bedrock valley. The presence of a "till shadow" causes the postglacial stream to follow a new course parallel to, and generally south of, the former channel (Coates, 1966, p. 1618).

\section{Moraine}

Moraines are mounds or ridges of unsorted and unstratified drift (mostly till) that melted out of the glacier during a pause (stillstand) in the glacier's retreat. These deposits generally do not contain high-yielding aquifers but may locally be underlain by confined aquifers, although none have been verified in this study area.

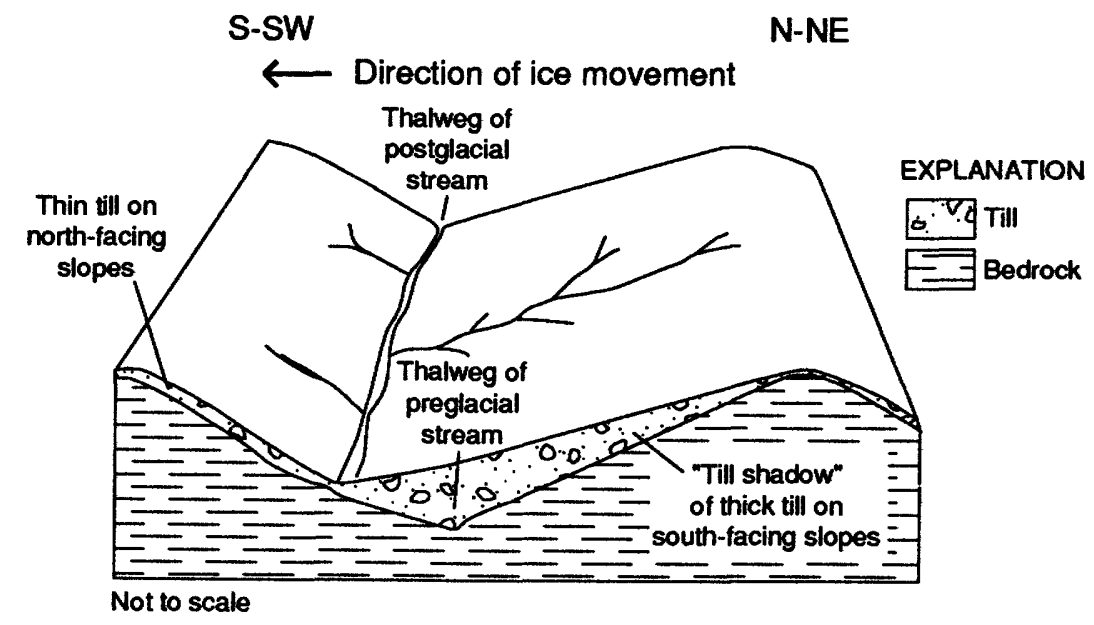

Figure 2.--Till-shadow hill within a typical upland tributary valley in Chenango County. (Modified from Coates, 1974, p. 224.) 


\section{Alluvium and Colluvium}

Alluvial and colluvial deposits are recent (postglacial) deposits and, therefore, overlie the glacial material in the valleys. Alluvium is deposited by modern streams, typically as alluvial fans, and consists of sorted clay, silt, sand, and gravel. Colluvium consists of loose and incoherent deposits of soil and rock, typically accumulated at the foot of a slope by rainwash or through downslope creep. These deposits have potentially good water-transmitting properties but are generally unsaturated because they are above the water table.

\section{Thickness}

Five thickness zones of unconsolidated deposits are delineated in plates 2A and 2B. Zone 1 (less than $5 \mathrm{ft}$ thick) is generally on hilltops and on the north-northeast-facing slopes, where bedrock is close to land surface. Till covers the bedrock uplands except where isolated pockets of sand and gravel were deposited during deglaciation. Zone 2 ( 5 to less than $50 \mathrm{ft}$ thick) is generally on the uplands and on hillsides. Zone 3 (50 to less than $100 \mathrm{ft}$ thick) occurs in kame terraces along the valley sides and in till-shadow hills (fig. 2). Zone 4 (100 to less than $200 \mathrm{ft}$ thick) is in the major valleys; zone 3 lies along each major valley flank, and zone 5 (equal or greater than $200 \mathrm{ft}$ thick), if present, is within the center of the valley. The major valleys may contain areas of zone 5 in addition to those shown, although present data are insufficient to determine their locations.

\section{Potential Well Yields from Valley-Fill Aquifers}

Plates 3A and 3B delineate four zones of potential well yields in the valley-fill aquifers. The most productive unconsolidated aquifers are in the major valleys and consist of coarse sand and gravel deposited by glacial meltwater streams. Valleys with high-yielding aquifers include the Chenango River, Unadilla River, Susquehanna River, Otselic River, Genegantslet Creek, and Mud Creek.

Some minor valleys that are tributary to the major valleys could also contain productive aquifers, but data are insufficientt to permit their delineation. The uplands and tributary valleys, particularly those that trend east-west, contain no major sand and gravel aquifers but may contain isolated pockets of sand and gravel that could serve as localized aquifers, but more data would be needed to verify their locations.

Zone 1 (less than $100 \mathrm{gal} / \mathrm{min}$ ) represents deposits of fine sand, silt, and clay in the major and minor valleys. Zone 2 (100 to less than 1,500 $\mathrm{gal} / \mathrm{min}$ ) also occurs in the valleys but represents more permeable deposits of sand and gravel. Zone $3(1,500$ to less than $3,500 \mathrm{gal} / \mathrm{min})$ is found primarily in the major valleys and represents mostly kame terraces and kame deltas that flank the valley sides. Kame terraces and kame deltas contain potentially high-yielding aquifer material but are largely unsaturated. In some areas, zone 1 and zone 3 are adjacent to one another. The abrupt change in thickness reflects the steep bedrock slope, which is a result of glacial erosion in the valleys. In such areas, zone 2 lies between zones 1 and 3 but is not shown because it would be extremely narrow, and the boundary location is uncertain. Zone 4 (equal to or greater than 3,500 $\mathrm{gal} / \mathrm{min}$ ) lies within the major valleys where the saturated thickness exceeds $40 \mathrm{ft}$ and the aquifer is in hydraulic connection with a stream whose drainage area exceeds $200 \mathrm{mi}^{2}$. Four areas in the lower Chenango River Valley meet these conditions (pl. 3B). 


\section{SUMMARY}

Unconsolidated deposits in Chenango County are thinnest on hilltops and north-northeast-facing slopes, and thickest in the major valleys. Hills with thick till on their south-southwest slopes (till shadows) occur in the uplands. The location of till shadows on such hills results from the direction of glacier movement relative to the axis of the upland tributary valleys.

The highest yielding aquifers in the county are the coarse sand and gravel valley-fill deposits that occupy the major valleys. Well yields in these valleys range from 100 to more than 3,500 $\mathrm{gal} / \mathrm{min}$. Ground-water supply in the upland areas is derived from bedrock, but well yields vary locally, depending on the degree of bedrock fracturing. Localized confined aquifers are present in all major valleys, but no data on their yields are available.

\section{SELECTED REFERENCES}

Bugliosi, E.F., Trudell, R.A., and Casey, G.D., 1988 , Potential yields of wells in unconsolidated aquifers in upstate New YorkHudson-Mohawk sheet: U.S. Geological Survey Water-Resources Investigations Report 87-4275, scale 1:250,000.

Cadwell, D.H., 1972, Glacial geology of the northern Chenango River Valley in McLelland, James (ed.): New York State Geological Association Field Trip Guidebook, 44th annual meeting, Utica, N.Y., p. D1-D4.

1972, Late Wisconsinan deglaciation chronology of the Chenango River Valley and vicinity, New York: State University of New York at Binghamton, unpublished Ph.D. thesis, $102 \mathrm{p}$.

1981, Glacial geology of the Chenango River Valley between Binghamton and Norwich, New York, in Enos, Paul, ed., New York State Geological Association Guidebook for field trips in south-central New York: Binghamton, N.Y., p. 97-114.

Cadwell, D.H., and Dineen, R.J. (eds.), 1987, Surficial geologic map of New YorkHudson-Mohawk sheet: Albany, N.Y., New York State Museum and Science Service Map and Chart Series no. 40, 1 sheet, $1: 250,000$ scale.
Coates, D.R., 1966, Glaciated Appalachian Plateau-till shadows on hills: Science, $v$. 152, p. $1617-1619$.

1974, Reappraisal of the glaciated Appalachian Plateau, in Coates, D. R., ed., Glacial geomorphology - chapter 8, publications in geomorphology: Binghamton, N.Y., State University of New York, p. 205-243.

Crandall, Leslie, 1985, Soil survey of Chenango County, New York: U.S. Department of Agriculture, Soil Conservation Service, $191 \mathrm{p}$.

Hollyday, E.F., 1969, An appraisal of the groundwater resources of the Susquehanna River Basin in New York: U.S. Geological Survey Open-File Report, 52 p.

Lumia, Richard, 1991, Regionalization of flood discharges on rural, unregulated streams in New York excluding Long Island: U.S. Geological Survey Water-Resources Investigations Report 90-4197, 119 p.

Lyford, F.P., Dysart, J.E., Randall, A.D., and Kontis, A.L., 1984, Glacial aquifer systems in the northeastern United States-a study plan: U.S. Geological Survey Open-File Report 83-0928, 33 p. 


\section{SELECTED REFERENCES (continued)}

MacNish, R.D., and Randall, A.D., 1982, Stratified-drift aquifers in the Susquehanna River Basin, New York: New York State Department of Environmental Conservation, Bulletin $75,68 \mathrm{p}$.

Miller, T.S., 1987, Unconsolidated aquifers in upstate New York-Finger Lakes sheet: U.S. Geological Survey Water-Resources Investigations Report 87-4122, scale $1: 250,000$.

Muller, E.H., and Cadwell, D.H. (eds.), Surficial geologic map of New York-Finger Lakes sheet: Albany, N.Y., New York State Museum and Science Service Map and Chart Series no. 40, 1 sheet, 1:250,000 scale.
Randall, A.D., 1972, Records of wells and test borings in the Susquehanna River Basin, New York: New York State Department of Environmental Conservation Bulletin 69, $92 \mathrm{p}$.

1978, Infiltration from tributary streams in the Susquehanna River Basin, New York: U.S. Geological Survey Journal of Research, v. 6, no. 3, May-June 1978, p. 285-297.

Reynolds, R. J., and Brown, G. A., 1984, Hydrogeologic appraisal of a stratified-drift aquifer near Smyrna, Chenango County, New York: U.S. Geological Survey WaterResources Investigations Report 84-4029, $53 \mathrm{p}$. 
Table 1.--Records of wells and test holes.

Explanation of Column Headings

Well number:

Location:

Owner:

Date drilled:

Altitude of land surface:

Well depth:

Casing depth:

Depth to bedrock:

Geologic unit:

Water level:

Yields:

Remarks:
Assigned according to the U.S. Geological Survey's sequential county numbering system for New York State. Wells are listed in order of increasing well number.

Latitude and longitude, in degrees, minutes, and seconds, were digitized from 7.5minute, 1:24,000-scale topographic maps unless derived from geologic logs. Locations of wells are shown on plates 1A and 1B. Accuracy of latitude and longitude as stated in the "Accuracy" column are as follows:

$$
S=1 \text { second } \quad F=5 \text { seconds } \quad T=10 \text { seconds }
$$

Owner of well or test hole at time of data collection:

NYSDOT $=$ New York State Department of Transportation.

NYSEG $=$ New York State Electric and Gas.

USGS = U.S. Geological Survey.

Completion date of well or test hole listed as month, day, and year, if known, otherwise month and year, or year.

Altitude of land surface above sea level was estimated to the nearest foot from 7.5minute, 1:24,000 scale topographic maps (contour interval $20 \mathrm{ft}$ ) for domestic wells, unless derived from geologic logs.

Total depth drilled, in feet below land surface.

Total length of solid casing, in feet below land surface, except as noted. The information was derived from geologic logs and driller's records.

Approximately equal to casing length for bedrock wells unless otherwise specified in a geologic log. Bedrock depths are shown on plates 3A and 3B.

Geologic unit that the well taps or test hole is terminated in:

QRNR Undifferentiated glacial sediments from the Quaternary Epoch

SDGV Sand and gravel (undifferentiated)

GRVL Gravel (undifferentiated)

LAKE Lacustrine deposits (silt, clay, fine sand)

OTSH Outwash (mostly sorted sand and gravel)

KMTC Ice-contact deposits (poorly to well-sorted sand and gravel)

BDRK Devonian bedrock (shale, siltstone, sandstone, or limestone)

Numbers show water levels to the nearest foot below land surface under static (nonpumping) conditions, except as noted.

Reported in gallons per minute ( $\mathrm{gal} / \mathrm{min}$ ) from pumping tests of observation wells and test holes, or as bail-test estimates from drillers of domestic wells.

Symbols or expressions used to abbreviate data.

$\begin{array}{llll}\text { WL } & \text { water level } & \text { BR } & \text { bedrock } \\ \text { DD } & \text { drawdown } & \text { SH } & \text { shale } \\ \text { LSD } & \text { land-surface datum } & \text { STS } & \text { siltstone } \\ \text { GPM } & \text { gallons per minute } & \text { SS } & \text { sandstone } \\ \text { MG/L } & \text { milligrams per liter } & \text { LS } & \text { limestone } \\ \text { HP } & \text { hardpan (possibly till) } & ? & \text { data not verified }\end{array}$


Table 1.--Records of wells and test holes.

[Dashes indicate no data. Locations shown on pls. 1A, 1B.]

\begin{tabular}{|c|c|c|c|c|c|c|c|c|c|c|}
\hline \multirow[b]{2}{*}{$\begin{array}{c}\text { Well } \\
\text { number }\end{array}$} & \multicolumn{3}{|c|}{ Location } & \multirow[b]{2}{*}{ Owner } & \multirow[b]{2}{*}{$\begin{array}{c}\text { Date } \\
\text { drilled }\end{array}$} & \multirow{2}{*}{$\begin{array}{c}\text { Altitude } \\
\text { of land } \\
\text { surface } \\
\text { (feet) }\end{array}$} & \multirow[b]{2}{*}{$\begin{array}{l}\text { Well } \\
\text { depth } \\
\text { (feet) }\end{array}$} & \multirow[b]{2}{*}{$\begin{array}{l}\text { Casing } \\
\text { depth } \\
\text { (feet) }\end{array}$} & \multirow{2}{*}{$\begin{array}{l}\text { Depth } \\
\text { to } \\
\text { bedrock } \\
\text { (feet) }\end{array}$} & \multirow[b]{2}{*}{$\begin{array}{c}\text { Geologic } \\
\text { unit }\end{array}$} \\
\hline & $\begin{array}{l}\text { Lati- } \\
\text { tude } \\
\ldots, n\end{array}$ & $\begin{array}{c}\text { Longi- } \\
\text { tude } \\
\ldots n\end{array}$ & $\begin{array}{c}\text { Accu- } \\
\text { racy }\end{array}$ & & & & & & & \\
\hline 1 & 423155 & 0752530 & $S$ & USGS & 1934 & 1452 & 8 & -- & -- & ORNR \\
\hline 2 & 424107 & 0751926 & $S$ & Kraft Food & 1963 & 1118 & 61 & 41 & -- & SDGV \\
\hline 3 & 424106 & 0751927 & $\mathbf{S}$ & Kraft Food & 1965 & 1112 & 61 & 34 & -. & SDGV \\
\hline 4 & 424106 & 0751927 & $S$ & Kraft Food & 1964 & 1112 & 54 & - & -- & SDGV \\
\hline 5 & 424107 & 0751927 & $\mathbf{S}$ & Kraft Food & 1964 & 1116 & 58 & 58 & -- & SDGV \\
\hline 6 & 424108 & 0751925 & $\mathbf{S}$ & Kraft Food & 1964 & 1120 & 50 & 50 & - & -- \\
\hline 7 & 424105 & 0751927 & $S$ & Kraft Food & 1964 & 1112 & 60 & 60 & -- & SDGV \\
\hline 8 & 424107 & 0751925 & $S$ & Kraft Food & 1964 & 1120 & 60 & 40 & -- & SDGV \\
\hline 9 & 424105 & 0751924 & $S$ & Kraft Food & 1964 & 1112 & 59 & 59 & - & QRNR \\
\hline 10 & 424107 & 0751926 & $\mathbf{S}$ & Kraft Food & 1964 & 1118 & 120 & 120 & -- & SDGV \\
\hline 11 & 421556 & 0752816 & $\mathbf{F}$ & Bullis & $10-15-65$ & 980 & 17 & 13 & -- & SDGV \\
\hline 12 & 422359 & 0752339 & $\mathbf{F}$ & NYSDOT & -- & 999 & 77 & -- & -- & QRNR \\
\hline 13 & 422352 & 0752347 & F & NYSDOT & -- & 1071 & 58 & -- & -- & QRNR \\
\hline 14 & 421157 & 0753519 & - & Glover & 1918 & 957 & 148 & 148 & -- & QRNR \\
\hline 15 & 421202 & 0753302 & - & Grobsky & -- & 1044 & 100 & 100 & -- & QRNR \\
\hline 16 & 421415 & 0753051 & - & Nelson & 1963 & 1005 & 195 & 195 & -- & QRNR \\
\hline 17 & 421416 & 0753016 & - & -. & 1942 & 1020 & 280 & 280 & -- & QRNR \\
\hline 18 & 421416 & 0753019 & - & Shupe Oldsmobile & 1958 & 1015 & 277 & 277 & -- & QRNR \\
\hline 19 & 421417 & 0753049 & - & Stewart & 1967 & 1000 & 226 & 226 & -- & QRNR \\
\hline 20 & 421419 & 0753028 & - & Enstrom & 1950 & 1010 & 287 & 287 & -- & QRNR \\
\hline 21 & 421421 & 0753028 & - & Tracy & 1953 & 1010 & 268 & 268 & -- & QRNR \\
\hline 22 & 421437 & 0752916 & $\mathrm{~T}$ & Seaman & 1965 & 987 & 256 & 256 & -- & QRNR \\
\hline 23 & 421441 & 0753016 & - & -- & 1942 & 1020 & 280 & 280 & -- & - \\
\hline 24 & 421444 & 0755012 & - & Clark & - & 912 & 55 & 55 & -- & QRNR \\
\hline 25 & 421511 & 0754923 & - & Gurley & 1961 & 912 & 74 & 74 & -- & QRNR \\
\hline 26 & 421530 & 0754916 & - & Chenango Trailer Park & 1952 & 905 & 84 & 50 & 50 & BDRK \\
\hline 27 & 421602 & 0754854 & - & Sport & 1962 & 920 & 75 & 32 & 32 & BDRK \\
\hline 28 & 421608 & 0752813 & $\mathbf{S}$ & Wilcox & 1930 & 992 & 252 & 250 & 250 & BDRK \\
\hline 29 & 421632 & 0752848 & $\mathbf{F}$ & NYSEG & 1950 & 978 & 40 & 36 & -- & SDGV \\
\hline 30 & 421637 & 0754845 & - & Taylor & 1954 & 950 & 110 & 40 & 40 & BDRK \\
\hline 31 & 421652 & 0754840 & - & Genegantslet Motel & 1956 & 942 & 100 & 50 & 50 & BDRK \\
\hline 32 & 421708 & 0752842 & - & Curtis & 1949 & 996 & 130 & 130 & -- & QRNR \\
\hline 33 & 421713 & 0752845 & - & Sipple & 1929 & 995 & 101 & 101 & -- & SDGV \\
\hline 34 & 421749 & 0752534 & - & Gifford & - & 985 & 109 & 109 & -- & -- \\
\hline 35 & 421751 & 0752532 & $S$ & Punako & 1951 & 990 & 180 & 180 & -- & QRNR \\
\hline 36 & 421758 & 0754822 & - & Capra & 1940 & 918 & 78 & 78 & -- & QRNR \\
\hline 37 & 421803 & 0752622 & $S$ & Lewis, Frank Inc. & 1945 & 1000 & 62 & 56 & - & SDGV \\
\hline 38 & 421806 & 0752725 & $S$ & Faigle & 1965 & 992 & 218 & 200 & 200 & BDRK \\
\hline 39 & 421833 & 0754741 & - & Holdredge & 1959 & 915 & 56 & 56 & -- & QRNR \\
\hline 40 & 421853 & 0754556 & $S$ & Gordon & 1960 & 1022 & 204 & 104 & 104 & BDRK \\
\hline 41 & 421907 & 0754406 & $\mathbf{S}$ & Robinson & 1962 & 1065 & 85 & -- & 82 & ORNR \\
\hline 42 & 421908 & 0754339 & $S$ & Fairchild & 1950 & 1080 & 78 & 63 & 63 & BDRK \\
\hline 43 & 421958 & 0754545 & - & Greene Village & 1906 & 910 & 159 & - & - & QRNR \\
\hline 44 & 421959 & 0754545 & $\mathbf{S}$ & Greene Village & 1959 & 905 & 170 & 162 & 162 & BDRK \\
\hline 45 & 422026 & 0754401 & - & Coleman & 1961 & 1010 & 194 & 40 & 40 & BDRK \\
\hline 46 & 422029 & 0754428 & - & Beach & 1962 & 925 & 180 & 180 & -- & QRNR \\
\hline 47 & 422036 & 0754346 & - & Keller & 1966 & 1018 & 145 & 19 & 19 & BDRK \\
\hline 48 & 422043 & 0754219 & - & Wright & 1958 & 930 & 70 & 20 & 20 & BDRK \\
\hline 49 & 422045 & 0754207 & - & Hall & 1962 & 938 & 80 & 80 & -- & QRNR \\
\hline 50 & 422054 & 0754155 & - & Hobert & -- & 945 & 90 & 90 & -- & QRNR \\
\hline
\end{tabular}


Table 1.--Records of wells and test holes.

[Dashes indicate no data. Locations shown on pls. 1A, 1B.]

\begin{tabular}{|c|c|c|c|c|}
\hline \multirow{2}{*}{$\begin{array}{c}\text { Well } \\
\text { number }\end{array}$} & \multicolumn{2}{|c|}{ Water level } & \multirow{2}{*}{$\begin{array}{c}\text { Yield } \\
\text { (gal/min) }\end{array}$} & \multirow{2}{*}{$\begin{array}{l}\text { Remarks } \\
\text { (Depths of geologic units given in feet below land surface) }\end{array}$} \\
\hline & feet & date & & \\
\hline 1 & 1 & $01-01-51$ & -- & --Weekly water-level readings from 1934-1951. \\
\hline 2 & 17 & 09.63 & 407 & -- \\
\hline 3 & 11 & $06-65$ & 700 & -- \\
\hline 4 & 22 & $12-64$ & 600 & -- \\
\hline 5 & 10 & $05-64$ & - & 0-34 sand \& silt, some clay; $34-42$ sand; $42-54$ sand, gravel, some silt; $54-58$ till. \\
\hline 6 & -- & -- & -- & -- \\
\hline 7 & 16 & $07-64$ & -- & $\begin{array}{l}0-17 \text { sand \& silt; } 17-30 \text { gravel w/silt; } 30-39 \text { sand; } 39-45 \text { gravel w/sand; } 45-60 \\
\text { sand \& silt. }\end{array}$ \\
\hline 8 & 18 & $07-64$ & - & 0-36 sand, w/silt \& gravel; 36-45 sand \& gravel; 45-60 sand, silt, \& clay. \\
\hline 9 & -- & - & - & 0-29 sand, silt, \& gravel; $29-49$ sand, silt, \& clay; $49-59$ sand, some silt \& clay. \\
\hline 10 & 12 & $06-64$ & -- & $\begin{array}{l}0-40 \text { sand, some silt \& gravel; } 40-60 \text { sand \& gravel; } 60-117 \text { sand, silt, \& clay; } \\
117-120 \text { till. }\end{array}$ \\
\hline 11 & 9 & $11-30-65$ & -- & $\begin{array}{l}\text { 0-12 silty gravel; } 12-15 \text { gravel; } 15-17 \text { gravel, silt, \& clay. WL measured weekly } \\
\text { since } 1965 \text {. }\end{array}$ \\
\hline 12 & -- & -- & -- & $0-15$ silt; $15-25$ sand w/gravel; $25-40$ silt; $40-50$ sand \& gravel; $50-77$ fine sand. \\
\hline 13 & -- & -- & -- & 0-26 silt, sand \& gravel; $26-51$ sand, some stone; $51-58$ silt, sand \& gravel. \\
\hline 14 & -- & -- & 8 & 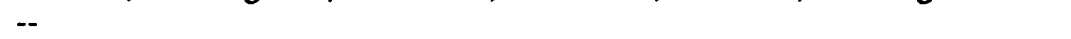 \\
\hline 15 & 50 & $03-64$ & 20 & -- \\
\hline 16 & -- & -- & 10 & 0-10 gravel; 10-190 fine sand; 190-195 gravel. \\
\hline 17 & 35 & $07-60$ & 20 & -- \\
\hline 18 & 31 & $10-58$ & 10 & -- \\
\hline 19 & 25 & $08-67$ & 30 & 0-5 gravel; 5-200 fine sand; 200-226 gravel. \\
\hline 20 & -- & -- & 10 & -- \\
\hline 21 & -- & -- & 12 & $0-30$ gravel; $30-200$ sand; $200-268$ fine sand. \\
\hline 22 & 20 & $10-65$ & 40 & $\begin{array}{l}\text { 0-30 gravel \& HP; } 30-70 \text { sand \& gravel; } 70-230 \text { clay; } 230-242 \text { sand \& gravel; } \\
242-256 \text { gravel. }\end{array}$ \\
\hline 23 & 35 & $07-60$ & 20 & -- \\
\hline 24 & 19 & $08-59$ & 12 & -- \\
\hline 25 & 10 & $10-61$ & 33 & 0-30 sand \& gravel; $30-65$ clay; $65-74$ black sand. \\
\hline 26 & 31 & 1952 & 22 & 0-50 gravel; 50-84 BR. \\
\hline 27 & -- & - & 30 & 0-10 gravel; $10-32$ clay; $32-75$ BR. Location approximate. \\
\hline 28 & 20 & $08-55$ & 5 & 0-10 gravel; $10-245$ fine sand; $245-250$ gravel; $250-252$ BR. \\
\hline 29 & 15 & $09-65$ & 7 & Pumped continuously. Location approximate. \\
\hline 30 & -- & -- & 10 & $0-40$ gravel; 40-110 BR. Location approximate. \\
\hline 31 & 20 & $06-56$ & 10 & 0-50 gravel; 50-100 BR. \\
\hline 32 & - & - & 48 & Penetrates sand, then clay, ends in pebbly gravel. \\
\hline 33 & 25 & $10-51$ & -- & 0-40 sediment; 40-97 fine sand; $97-103$ gravel. Water at 97 , no water at 103 . \\
\hline 34 & 20 & $06-67$ & -- & -- \\
\hline 35 & -- & -- & 15 & -- \\
\hline 36 & 14 & $06-59$ & 22 & -- \\
\hline 37 & 27 & $04-45$ & 300 & $0-62$ coarse sand \& gravel. \\
\hline 38 & 29 & $07-65$ & 15 & 0-198 fine sand; $198-200$ gravel; 200-218 BR. \\
\hline 39 & -- & - & 22 & 0-32 gravel; $32-54$ fine sand; 54-56 gravel. \\
\hline 40 & 44 & $06-60$ & 10 & - \\
\hline 41 & -- & -- & -- & $0-82$ gravel, then possibly till. No water. \\
\hline 42 & -- & -- & 16 & -- \\
\hline 43 & -- & -- & 330 & Pumped continuously. \\
\hline 44 & 18 & $05-59$ & 355 & 0-160 clay; $160-170$ small gravel. Hardness $130 \mathrm{MG} / \mathrm{L}$-auxillary well. \\
\hline 45 & -- & -- & 20 & 0-20 gravel; $20-40$ coarse gravel; $40-194 \mathrm{BR}$. \\
\hline 46 & -- & -- & 12 & -- \\
\hline 47 & 25 & $05-66$ & 20 & 0-19 gravel; 19-145 BR. \\
\hline 48 & -- & -- & 11 & - \\
\hline 49 & 31 & $12-62$ & 8 & - \\
\hline 50 & 30 & $07-62$ & 10 & -- \\
\hline
\end{tabular}


Table 1.--Records of wells and test holes--continued.

[Dashes indicate no data. Locations shown on pls. 1A, 1B.]

\begin{tabular}{|c|c|c|c|c|c|c|c|c|c|c|}
\hline \multirow[b]{2}{*}{$\begin{array}{c}\text { Well } \\
\text { number }\end{array}$} & \multicolumn{3}{|c|}{ Location } & \multirow[b]{2}{*}{ Owner } & \multirow[b]{2}{*}{$\begin{array}{c}\text { Date } \\
\text { drilled }\end{array}$} & \multirow{2}{*}{$\begin{array}{l}\text { Altitude } \\
\text { of land } \\
\text { surface } \\
\text { (feet) }\end{array}$} & \multirow[b]{2}{*}{$\begin{array}{l}\text { Well } \\
\text { depth } \\
\text { (feet) }\end{array}$} & \multirow[b]{2}{*}{$\begin{array}{l}\text { Casing } \\
\text { depth } \\
\text { (feet) }\end{array}$} & \multirow{2}{*}{$\begin{array}{l}\text { Depth } \\
\text { to } \\
\text { bedrock } \\
\text { (feet) }\end{array}$} & \multirow[b]{2}{*}{$\begin{array}{c}\text { Geologic } \\
\text { unit }\end{array}$} \\
\hline & $\begin{array}{l}\text { Lati- } \\
\text { tude } \\
\cdots n\end{array}$ & $\begin{array}{c}\text { Longi- } \\
\text { tude } \\
\text {... }\end{array}$ & $\begin{array}{l}\text { Accu- } \\
\text { racy }\end{array}$ & & & & & & & \\
\hline $\begin{array}{l}51 \\
52 \\
53 \\
54 \\
55\end{array}$ & $\begin{array}{l}422100 \\
422100 \\
422109 \\
422118 \\
422120\end{array}$ & $\begin{array}{l}0754411 \\
0754839 \\
0754320 \\
0754226 \\
0754235\end{array}$ & $\begin{array}{l}\text { F } \\
- \\
- \\
-\end{array}$ & $\begin{array}{l}\text { Rheubottom } \\
\text { Hollenbeck } \\
\text { Bennett } \\
\text { B\&B Build Supply } \\
\text { Rounds Brothers }\end{array}$ & $\begin{array}{l}1946 \\
1912 \\
1966 \\
1961 \\
1961\end{array}$ & $\begin{array}{r}923 \\
1003 \\
928 \\
938 \\
938\end{array}$ & $\begin{array}{r}125 \\
16 \\
50 \\
125 \\
96\end{array}$ & $\begin{array}{r}110 \\
- \\
50 \\
90 \\
60\end{array}$ & $\begin{array}{r}110 \\
-- \\
-- \\
90 \\
60\end{array}$ & $\begin{array}{l}\text { BDRK } \\
\text { QRNR } \\
\text { QRNR } \\
\text { BDRK } \\
\text { BDRK }\end{array}$ \\
\hline $\begin{array}{l}56 \\
57 \\
58 \\
59 \\
60\end{array}$ & $\begin{array}{l}422121 \\
422124 \\
422126 \\
422126 \\
422135\end{array}$ & $\begin{array}{l}0754231 \\
0754225 \\
0754136 \\
0754223 \\
0754118\end{array}$ & $\begin{array}{l}- \\
- \\
- \\
-\end{array}$ & $\begin{array}{l}\text { Harrington } \\
\text { Edgerton } \\
\text { Lobdell } \\
\text { Alger } \\
\text { Gridley }\end{array}$ & $\begin{array}{l}1956 \\
1963 \\
1958 \\
1962 \\
1956\end{array}$ & $\begin{array}{l}935 \\
938 \\
950 \\
940 \\
948\end{array}$ & $\begin{array}{l}91 \\
76 \\
80 \\
74 \\
61\end{array}$ & $\begin{array}{l}91 \\
70 \\
50 \\
55 \\
25\end{array}$ & $\begin{array}{l}-- \\
70 \\
50 \\
55 \\
25\end{array}$ & $\begin{array}{l}\text { QRNR } \\
\text { BDRK } \\
\text { BDRK } \\
\text { BDRK } \\
\text { BDRK }\end{array}$ \\
\hline $\begin{array}{l}61 \\
62 \\
63 \\
64 \\
65\end{array}$ & $\begin{array}{l}422139 \\
422148 \\
422152 \\
422200 \\
422203\end{array}$ & $\begin{array}{l}0754113 \\
0754105 \\
0754053 \\
0754042 \\
0753838\end{array}$ & $\begin{array}{l}- \\
- \\
-\end{array}$ & $\begin{array}{l}\text { Davis } \\
\text { Peterson } \\
\text { Brisben Diner } \\
\text { Alger } \\
\text { Meade }\end{array}$ & $\begin{array}{l}1964 \\
-- \\
1964 \\
1958 \\
1965\end{array}$ & $\begin{array}{l}940 \\
950 \\
935 \\
938 \\
965\end{array}$ & $\begin{array}{r}62 \\
69 \\
90 \\
92 \\
131\end{array}$ & $\begin{array}{l}15 \\
41 \\
90 \\
90 \\
32\end{array}$ & $\begin{array}{l}15 \\
41 \\
- \\
90 \\
32\end{array}$ & $\begin{array}{l}\text { BDRK } \\
\text { BDRK } \\
\text { QRNR } \\
\text { BDRK } \\
\text { BDRK }\end{array}$ \\
\hline $\begin{array}{l}66 \\
67 \\
68 \\
70 \\
71\end{array}$ & $\begin{array}{l}422206 \\
422243 \\
422313 \\
422338 \\
422341\end{array}$ & $\begin{array}{l}0753858 \\
0753810 \\
0752423 \\
0754828 \\
0754831\end{array}$ & $\begin{array}{l}\text { S } \\
- \\
\text { F }\end{array}$ & $\begin{array}{l}\text { Meade } \\
\text { Gerhardt } \\
\text { Thomas } \\
\text { Auwarter } \\
\text { Thatcher }\end{array}$ & $\begin{array}{l}1963 \\
-- \\
1965 \\
1954 \\
1954\end{array}$ & $\begin{array}{r}942 \\
970 \\
1021 \\
1020 \\
1024\end{array}$ & $\begin{array}{r}65 \\
187 \\
119 \\
86 \\
62\end{array}$ & $\begin{array}{r}30 \\
187 \\
119 \\
65 \\
53\end{array}$ & $\begin{array}{l}30 \\
\cdots \\
65 \\
53\end{array}$ & \begin{tabular}{l} 
BDRK \\
QRNR \\
\hdashline- \\
BDRK \\
BDRK
\end{tabular} \\
\hline $\begin{array}{l}72 \\
73 \\
74 \\
75\end{array}$ & $\begin{array}{l}422403 \\
422405 \\
422417 \\
422418\end{array}$ & $\begin{array}{l}0752341 \\
0752335 \\
0754836 \\
0754828\end{array}$ & $\bar{s}$ & $\begin{array}{l}\text { Knetgen } \\
\text { Knetgen } \\
\text { Boardman } \\
\text { USGS }\end{array}$ & $\begin{array}{l}1964 \\
1953 \\
1965 \\
1967\end{array}$ & $\begin{array}{l}1045 \\
1025 \\
1060 \\
1047\end{array}$ & $\begin{array}{l}25 \\
24 \\
45 \\
25\end{array}$ & $\begin{array}{l}24 \\
24 \\
14 \\
25\end{array}$ & $\begin{array}{c}-- \\
-- \\
14 \\
--\end{array}$ & $\begin{array}{l}\text { QRNR } \\
\text { QRNR } \\
\text { BDRK } \\
\text { ICNC }\end{array}$ \\
\hline 76 & 422418 & 0754834 & $S$ & Urda & 1966 & 1061 & 24 & 24 & - & QRNR \\
\hline $\begin{array}{l}77 \\
78 \\
79 \\
80 \\
81\end{array}$ & $\begin{array}{l}422419 \\
422431 \\
422453 \\
422501 \\
422503\end{array}$ & $\begin{array}{l}0754836 \\
0752256 \\
0753619 \\
0752251 \\
0752249\end{array}$ & $\begin{array}{l}S \\
S \\
F \\
- \\
-\end{array}$ & $\begin{array}{l}\text { Martin } \\
\text { Stone } \\
\text { Roach } \\
\text { Thomas } \\
\text { Copper }\end{array}$ & $\begin{array}{l}1967 \\
1963 \\
1947 \\
1942 \\
1964\end{array}$ & $\begin{array}{l}1077 \\
1045 \\
1329 \\
1040 \\
1040\end{array}$ & $\begin{array}{r}440 \\
315 \\
179 \\
25 \\
27\end{array}$ & $\begin{array}{r}40 \\
270 \\
103 \\
21 \\
23\end{array}$ & $\begin{array}{r}40 \\
270 \\
103 \\
-- \\
--\end{array}$ & $\begin{array}{l}\text { BDRK } \\
\text { BDRK } \\
\text { BDRK } \\
\text { QRNR } \\
\text { QRNR }\end{array}$ \\
\hline $\begin{array}{l}82 \\
83 \\
84 \\
85 \\
86\end{array}$ & $\begin{array}{l}422513 \\
422520 \\
422536 \\
422552 \\
422552\end{array}$ & $\begin{array}{l}0753814 \\
0753807 \\
0754910 \\
0754914 \\
0754914\end{array}$ & $\begin{array}{l}- \\
S \\
S\end{array}$ & $\begin{array}{l}\text { Youngs } \\
\text { Colton } \\
\text { Pratt } \\
\text { Moore } \\
\text { Moore }\end{array}$ & $\begin{array}{c}1964 \\
1965 \\
1964 \\
1966 \\
--\end{array}$ & $\begin{array}{r}970 \\
972 \\
1305 \\
1349 \\
1350\end{array}$ & $\begin{array}{r}68 \\
22 \\
134 \\
128 \\
20\end{array}$ & $\begin{array}{r}68 \\
22 \\
134 \\
123 \\
--\end{array}$ & $\begin{array}{r}-- \\
-- \\
-- \\
123 \\
--\end{array}$ & $\begin{array}{l}\text { OTSH } \\
\text { OTSH } \\
\text { QRNR } \\
\text { BDRK } \\
\text { SDGV }\end{array}$ \\
\hline $\begin{array}{l}87 \\
88 \\
89 \\
90\end{array}$ & $\begin{array}{l}422559 \\
422601 \\
422633 \\
422645\end{array}$ & $\begin{array}{l}0752314 \\
0752314 \\
0755109 \\
0753540\end{array}$ & $\dot{-}$ & $\begin{array}{l}\text { Sprague } \\
\text { Asa } \\
\text { Fry } \\
\text { Oxford Village }\end{array}$ & $\begin{array}{c}1947 \\
1965 \\
1940 \\
11-21-64\end{array}$ & $\begin{array}{r}1040 \\
1045 \\
1462 \\
968\end{array}$ & $\begin{array}{r}40 \\
70 \\
185 \\
97\end{array}$ & $\begin{array}{r}40 \\
70 \\
34 \\
--\end{array}$ & $\overline{-}$ & $\begin{array}{l}\text { QRNR } \\
\text { QRNR } \\
\text { BDRK } \\
\text { OTSH }\end{array}$ \\
\hline 91 & 422649 & 0752322 & - & Hadeler & 1920 & 1039 & 22 & 22 & -- & QRNR \\
\hline $\begin{array}{l}92 \\
93 \\
94 \\
95 \\
96\end{array}$ & $\begin{array}{l}422650 \\
422651 \\
422658 \\
422710 \\
422715\end{array}$ & $\begin{array}{l}0752321 \\
0755005 \\
0755009 \\
0753507 \\
0753457\end{array}$ & $\begin{array}{l}- \\
- \\
s\end{array}$ & $\begin{array}{l}\text { Mickle } \\
\text { Harris } \\
\text { Grover } \\
\text { Hackett Carwash } \\
\text { Langer }\end{array}$ & $\begin{array}{l}1960 \\
1954 \\
1966 \\
1965 \\
1964\end{array}$ & $\begin{array}{r}1035 \\
1390 \\
1436 \\
1000 \\
985\end{array}$ & $\begin{array}{r}118 \\
118 \\
145 \\
69 \\
78\end{array}$ & $\begin{array}{r}118 \\
118 \\
140 \\
-- \\
78\end{array}$ & $\begin{array}{r}-- \\
-- \\
140 \\
-- \\
--\end{array}$ & $\begin{array}{l}\text { QRNR } \\
\text { SDGV } \\
\text { BDRK } \\
\text { QRNR } \\
\text { QRNR }\end{array}$ \\
\hline $\begin{array}{r}97 \\
98 \\
99 \\
100 \\
101\end{array}$ & $\begin{array}{l}422723 \\
422728 \\
422738 \\
422740 \\
422741\end{array}$ & $\begin{array}{l}0753452 \\
0752347 \\
0752402 \\
0752405 \\
0753456\end{array}$ & $i$ & $\begin{array}{l}\text { Barber } \\
\text { Miller } \\
\text { Fancher } \\
\text { Clark } \\
\text { Ricks }\end{array}$ & $\begin{array}{l}1944 \\
1964 \\
1963 \\
1958 \\
1961\end{array}$ & $\begin{array}{r}985 \\
1045 \\
1060 \\
1045 \\
1010\end{array}$ & $\begin{array}{r}90 \\
150 \\
126 \\
148 \\
40\end{array}$ & $\begin{array}{r}90 \\
150 \\
126 \\
144 \\
31\end{array}$ & $\begin{array}{c}-- \\
-- \\
-- \\
31\end{array}$ & $\begin{array}{l}\text { QRNR } \\
\text { QRNR } \\
\text { QRNR } \\
\text { QRNR } \\
\text { BDRK }\end{array}$ \\
\hline
\end{tabular}


Table 1.--Records of wells and test holes--continued.

[Dashes indicate no data. Locations shown on pls. 1A, 1B.]

\begin{tabular}{|c|c|c|c|c|}
\hline \multirow{2}{*}{$\begin{array}{c}\text { Well } \\
\text { number }\end{array}$} & \multicolumn{2}{|c|}{ Water level } & \multirow{2}{*}{$\begin{array}{l}\text { Yield } \\
\text { (gal/min) }\end{array}$} & \multirow{2}{*}{$\begin{array}{c}\text { Remarks } \\
\text { (Depths of geologic units given in feet below land surface) }\end{array}$} \\
\hline & feet & date & & \\
\hline $\begin{array}{l}51 \\
52 \\
53 \\
54 \\
55\end{array}$ & $\begin{array}{r}20 \\
11 \\
-- \\
30 \\
30\end{array}$ & $\begin{array}{c}07-65 \\
07-66 \\
-- \\
1961 \\
09-61\end{array}$ & $\begin{array}{r}8 \\
10 \\
250 \\
10\end{array}$ & $\begin{array}{l}\text { 0-20 gravel; } 20-110 \text { clay \& stones; } 110-125 \text { BR. } \\
-- \\
\text { 0-90 gravel; } 90-125 \text { BR. } \\
--\end{array}$ \\
\hline $\begin{array}{l}56 \\
57 \\
58 \\
59 \\
60\end{array}$ & $\begin{array}{l}-- \\
15 \\
17 \\
25\end{array}$ & $\begin{array}{l}-- \\
06-58 \\
07-66 \\
07-56\end{array}$ & $\begin{array}{r}22 \\
16 \\
8 \\
8 \\
5\end{array}$ & $\begin{array}{l}\text { 0-70 coarse gravel; 70-76 BR. } \\
\text { 0-50 black sand, some cobbles; 50-80 BR. } \\
\text { 0-40 dark gravel, some stones; 40-55 clay; 55-74 BR. } \\
\text {-- }\end{array}$ \\
\hline $\begin{array}{l}61 \\
62 \\
63 \\
64 \\
65\end{array}$ & $\begin{array}{r}20 \\
-- \\
35 \\
--\end{array}$ & $\begin{array}{c}09-64 \\
-- \\
06-64 \\
10-58 \\
--\end{array}$ & $\begin{array}{r}25 \\
10 \\
8 \\
30 \\
20\end{array}$ & $\begin{array}{l}\text { 0-15 sand \& gravel; } 15-62 \mathrm{BR} \text {. } \\
0-35 \text { sand; } 35-41 \text { clay; } 41-69 \text { blue } \mathrm{SH} . \\
\text { 0-90 sand \& gravel; } 90-92 \mathrm{BR} \text {. } \\
\text { 0-32 gravel; 32-131 BR. }\end{array}$ \\
\hline $\begin{array}{l}66 \\
67 \\
68 \\
70 \\
71\end{array}$ & $\begin{array}{l}-- \\
30 \\
-- \\
--\end{array}$ & $\begin{array}{l}-- \\
04-65 \\
-- \\
--\end{array}$ & $\begin{array}{r}20 \\
-- \\
15 \\
5\end{array}$ & $\begin{array}{l}\text { 0-15 gravel; } 15-30 \text { fine sand; 30-65 BR. } \\
\text { Penetrates gravel, very fine sand, \& ends in sand. } \\
-- \\
\text { 0-65 dirty gravel, no water; } 65-86 \text { BR. } \\
\text { 0-53 dirty gravel, no water; } 53 \mathrm{BR} \text {. Iron } 5.3 \text {, Hardness } 99 \text {, total solids } 120 \mathrm{MG} / \mathrm{L} \text {. }\end{array}$ \\
\hline $\begin{array}{l}72 \\
73 \\
74 \\
75\end{array}$ & $\begin{array}{c}-- \\
8 \\
-- \\
7\end{array}$ & $\begin{array}{c}-- \\
09-54 \\
-- \\
10-67\end{array}$ & $\begin{array}{r}20 \\
25 \\
5\end{array}$ & $\begin{array}{l}\text { 0-15 clay, fine sand; } 15-25 \text { black gravel. Supplies motel and restaurant. } \\
\text { Location approximate. } \\
\text { Hardness } 103 \mathrm{MG} / \mathrm{L} \text {. } \\
0-17 \text { silty sand; } 17-23 \text { silt; } 23-25 \text { sand \& gravel. Water bearing from } 12-14 \text { \& } \\
16-17 \mathrm{ft} \text {. }\end{array}$ \\
\hline 76 & 12 & $10-67$ & 30 & - \\
\hline $\begin{array}{l}77 \\
78 \\
79 \\
80 \\
81\end{array}$ & $\begin{array}{r}20 \\
35 \\
29 \\
-- \\
--\end{array}$ & $\begin{array}{l}1967 \\
07-68 \\
09-47 \\
-- \\
--\end{array}$ & $\begin{array}{r}3 \\
7 \\
20 \\
10\end{array}$ & $\begin{array}{l}\text { Water enters below } 300 \mathrm{ft} \text {, Hardness } 51 \mathrm{MG} / \mathrm{L} \text {. } \\
0-20 \text { gravel; } 20-270 \text { clay; } 270 \mathrm{BR} \text {. Salt water. Flammable gas if pumped. } \\
0-103 \text { gravel and red clay. Location approximate. } \\
\text {-- } \\
0-10 \text { dirt \& gravel; } 10-20 \text { clay; } 20-27 \text { fine sand. }\end{array}$ \\
\hline $\begin{array}{l}82 \\
83 \\
84 \\
85 \\
86\end{array}$ & $\begin{array}{r}10 \\
-- \\
17 \\
8\end{array}$ & $\begin{array}{l}08-64 \\
-- \\
-- \\
12-66 \\
06-67\end{array}$ & $\begin{array}{r}5 \\
10 \\
15 \\
8\end{array}$ & $\begin{array}{l}0-15 \text { sand; } 15-50 \text { clay; } 50-60 \text { gravel; } 60-68 \text { black sand. } \\
0-22 \text { sand, HP, \& some gravel. } \\
0-12 \text { soil; } 12-30 \text { gravel; } 30-130 \text { HP; } 130-134 \text { gravel. } \\
--\end{array}$ \\
\hline $\begin{array}{l}87 \\
88 \\
89 \\
90\end{array}$ & $\begin{array}{l}-- \\
21 \\
30 \\
11\end{array}$ & $\begin{array}{c}-- \\
08-65 \\
08-40 \\
11-21-64\end{array}$ & $\begin{array}{r}30 \\
30 \\
-- \\
300\end{array}$ & $\begin{array}{l}- \\
- \\
\overline{0-10} \text { sand; } 10-25 \text { clay; } 25-63 \text { sand \& gravel; 63-68 HP; 68-97 sand \& gravel; } 97 \\
\text { HP. }\end{array}$ \\
\hline 91 & -- & -- & 25 & -- \\
\hline $\begin{array}{l}92 \\
93 \\
94 \\
95 \\
96\end{array}$ & $\begin{array}{r}25 \\
1 \\
60 \\
19 \\
15\end{array}$ & $\begin{array}{l}1960 \\
1954 \\
1966 \\
07-65 \\
1964\end{array}$ & $\begin{array}{l}30 \\
10 \\
34 \\
25\end{array}$ & $\begin{array}{l}\text { 0-10 sand \& gravel; } 10-110 \text { cobbles, hard drilling; } 110-118 \text { sand \& gravel. } \\
0-100 \text { till; } 100-118 \text { gravel. } \\
0-112 \text { till; } 112-115 \text { sand; } 115-140 \text { till; } 140 \text { BR. Sulfur odor, excess. iron. } \\
\text { 0-55 clay; } 55-60 \text { sand \& gravel; } 60-69 \text { gravel. } \\
\text {-- }\end{array}$ \\
\hline $\begin{array}{r}97 \\
98 \\
99 \\
100 \\
101\end{array}$ & $\begin{array}{r}30 \\
8 \\
-- \\
18 \\
--\end{array}$ & $\begin{array}{c}1964 \\
1964 \\
- \\
1958 \\
--\end{array}$ & $\begin{array}{r}100 \\
14 \\
25 \\
13 \\
30\end{array}$ & $\begin{array}{l}\text { 0-10 gravel; } 10-79 \text { mostly clay; } 79-90 \text { gravel. } \\
\text { 0-9 gravel; } 9-40 \text { fine sand; } 40-130 \text { gravel; } 130-150 \text { black sand \& gravel. } \\
\text { 0-100 clay; } 100-126 \text { sand \& gravel. } \\
\text { 0-60 gravel; } 60-109 \text { fine sand; } 109-119 \text { rock?; } 119-148 \text { sand \& gravel. } \\
\text {-- }\end{array}$ \\
\hline
\end{tabular}


Table 1.--Records of wells and test holes--continued.

[Dashes indicate no data. Locations shown on pls. 1A, 1B.]

\begin{tabular}{|c|c|c|c|c|c|c|c|c|c|c|}
\hline $\begin{array}{c}\text { Well } \\
\text { number }\end{array}$ & $\begin{array}{l}\text { Lati- } \\
\text { tude } \\
\cdots\end{array}$ & $\begin{array}{c}\text { Location } \\
\text { Longi- } \\
\text { tude } \\
\ldots \text {... }\end{array}$ & $\begin{array}{l}\text { Accu- } \\
\text { racy }\end{array}$ & Owner & $\begin{array}{c}\text { Date } \\
\text { drilled }\end{array}$ & $\begin{array}{l}\text { Altitude } \\
\text { of land } \\
\text { surface } \\
\text { (feet) }\end{array}$ & $\begin{array}{l}\text { Well } \\
\text { depth } \\
\text { (feet) }\end{array}$ & $\begin{array}{l}\text { Casing } \\
\text { depth } \\
\text { (feet) }\end{array}$ & $\begin{array}{l}\text { Depth } \\
\text { to } \\
\text { bedrock } \\
\text { (feet) }\end{array}$ & $\begin{array}{c}\text { Geologic } \\
\text { unit }\end{array}$ \\
\hline $\begin{array}{l}102 \\
103 \\
104 \\
105 \\
106\end{array}$ & $\begin{array}{l}422742 \\
422810 \\
422814 \\
422822 \\
422822\end{array}$ & $\begin{array}{l}0753427 \\
0753237 \\
0753236 \\
0753250 \\
0753252\end{array}$ & $\begin{array}{l}S \\
S \\
S \\
S \\
S\end{array}$ & $\begin{array}{l}\text { Mangels } \\
\text { Branham } \\
\text { Cunningham } \\
\text { Halfway Motel } \\
\text { Halfway Bar }\end{array}$ & $\begin{array}{c}1954 \\
1958 \\
1965 \\
1962 \\
09-01-66\end{array}$ & $\begin{array}{r}985 \\
985 \\
983 \\
988 \\
1000\end{array}$ & $\begin{array}{r}60 \\
169 \\
75 \\
178 \\
104\end{array}$ & $\begin{array}{r}- \\
169 \\
75 \\
168 \\
100\end{array}$ & $\begin{array}{r}-- \\
- \\
-- \\
168 \\
--\end{array}$ & $\begin{array}{l}\text { QRNR } \\
\text { SDGV } \\
\text { SDGV } \\
\text { BDRK } \\
\text { SDGV }\end{array}$ \\
\hline $\begin{array}{l}107 \\
108 \\
109 \\
110 \\
111\end{array}$ & $\begin{array}{l}422824 \\
422831 \\
422833 \\
422845 \\
422857\end{array}$ & $\begin{array}{l}0753253 \\
0753526 \\
0753242 \\
0753218 \\
0753231\end{array}$ & $\begin{array}{l}\text { S } \\
\text { F } \\
- \\
\text { S } \\
\text { S }\end{array}$ & $\begin{array}{l}\text { Myers } \\
\text { Strawn } \\
\text { Rothar } \\
\text { Kassler } \\
\text { Freds Inn }\end{array}$ & $\begin{array}{l}1962 \\
1945 \\
1960 \\
1956 \\
1964\end{array}$ & $\begin{array}{r}1003 \\
1194 \\
990 \\
1000 \\
1000\end{array}$ & $\begin{array}{r}85 \\
44 \\
255 \\
85 \\
88\end{array}$ & $\begin{array}{r}85 \\
39 \\
255 \\
85 \\
--\end{array}$ & $\begin{array}{c}-- \\
39 \\
-- \\
--\end{array}$ & $\begin{array}{l}\text { SDGV } \\
\text { BDRK } \\
\text { QRNR } \\
\text { SDGV } \\
\text { QRNR }\end{array}$ \\
\hline $\begin{array}{l}112 \\
113 \\
114 \\
115 \\
116\end{array}$ & $\begin{array}{l}422910 \\
422910 \\
422912 \\
422925 \\
422928\end{array}$ & $\begin{array}{l}0752412 \\
0753218 \\
0752413 \\
0753124 \\
0753206\end{array}$ & $\begin{array}{l}- \\
\text { S } \\
- \\
- \\
\end{array}$ & $\begin{array}{l}\text { Scott } \\
\text { Wickham } \\
\text { Pearsall } \\
\text { Palmer } \\
\text { Salisbury }\end{array}$ & $\begin{array}{l}1958 \\
1964 \\
1953 \\
1961 \\
1956\end{array}$ & $\begin{array}{l}1095 \\
1008 \\
1080 \\
1035 \\
1000\end{array}$ & $\begin{array}{r}152 \\
130 \\
150 \\
80 \\
69\end{array}$ & $\begin{array}{r}132 \\
130 \\
150 \\
15 \\
69\end{array}$ & $\begin{array}{r}132 \\
-- \\
-- \\
15 \\
--\end{array}$ & $\begin{array}{l}\text { BDRK } \\
\text { SDGV } \\
\text { QRNR } \\
\text { BDRK } \\
\text { SDGV }\end{array}$ \\
\hline $\begin{array}{l}117 \\
118 \\
119 \\
120 \\
121\end{array}$ & $\begin{array}{l}422938 \\
422941 \\
422951 \\
422953 \\
422954\end{array}$ & $\begin{array}{l}0754611 \\
0752401 \\
0754544 \\
0752404 \\
0753146\end{array}$ & $\begin{array}{l}\text { F } \\
- \\
\text { F } \\
- \\
\text { S }\end{array}$ & $\begin{array}{l}\text { Duel } \\
\text { Anderson } \\
\text { Haydn } \\
\text { Dull } \\
\text { Stern Ring }\end{array}$ & $\begin{array}{l}1955 \\
1954 \\
1950 \\
1964 \\
1960\end{array}$ & $\begin{array}{l}1414 \\
1050 \\
1310 \\
1090 \\
1035\end{array}$ & $\begin{array}{r}46 \\
160 \\
57 \\
270 \\
320\end{array}$ & $\begin{array}{r}22 \\
160 \\
11 \\
270 \\
--\end{array}$ & $\begin{array}{c}22 \\
-- \\
11 \\
-- \\
--\end{array}$ & $\begin{array}{l}\text { BDRK } \\
\text { QRNR } \\
\text { BDRK } \\
\text { QRNR } \\
\text { QRNR }\end{array}$ \\
\hline $\begin{array}{l}122 \\
123 \\
124 \\
125 \\
126\end{array}$ & $\begin{array}{l}422956 \\
422959 \\
423015 \\
423016 \\
423018\end{array}$ & $\begin{array}{l}0753159 \\
0753144 \\
0752419 \\
0754510 \\
0753125\end{array}$ & $\begin{array}{l}\text { S } \\
\text { S } \\
- \\
\text { F } \\
\text { S }\end{array}$ & $\begin{array}{l}\text { Johnson } \\
\text { Lonstein } \\
\text { Osborne } \\
\text { Decker }\end{array}$ & $\begin{array}{c}-- \\
1957 \\
-- \\
1946 \\
--\end{array}$ & $\begin{array}{l}1045 \\
1030 \\
1085 \\
1325 \\
1008\end{array}$ & $\begin{array}{r}90 \\
276 \\
26 \\
152 \\
190\end{array}$ & $\begin{array}{r}-- \\
276 \\
26 \\
85 \\
40\end{array}$ & $\begin{array}{l}-- \\
-- \\
-- \\
85 \\
40\end{array}$ & $\begin{array}{l}\text { SDGV } \\
\text { QRNR } \\
\text { BDRK } \\
\text { BDRK }\end{array}$ \\
\hline $\begin{array}{l}127 \\
128 \\
129 \\
130 \\
131\end{array}$ & $\begin{array}{l}423027 \\
423033 \\
423045 \\
423045 \\
423048\end{array}$ & $\begin{array}{l}0753113 \\
0753434 \\
0752345 \\
0752406 \\
0752404\end{array}$ & $\begin{array}{l}\mathbf{S} \\
- \\
- \\
- \\
-\end{array}$ & $\begin{array}{l}\text { Decker } \\
\text { Granger } \\
\text { Harris } \\
\text { Reed } \\
\text { Carpenter }\end{array}$ & $\begin{array}{c}1963 \\
1947 \\
- \\
1945 \\
1965\end{array}$ & $\begin{array}{r}990 \\
1610 \\
1042 \\
1050 \\
1055\end{array}$ & $\begin{array}{r}20 \\
127 \\
60 \\
208 \\
186\end{array}$ & $\begin{array}{r}18 \\
111 \\
60 \\
208 \\
186\end{array}$ & $\begin{array}{r}-- \\
111 \\
-- \\
-- \\
--\end{array}$ & $\begin{array}{l}\text { SDGV } \\
\text { BDRK } \\
\text { QRNR } \\
\text { QRNR } \\
\text { QRNR }\end{array}$ \\
\hline $\begin{array}{l}132 \\
133 \\
134 \\
135 \\
136\end{array}$ & $\begin{array}{l}423057 \\
423123 \\
423128 \\
423139 \\
423152\end{array}$ & $\begin{array}{l}0753114 \\
0753103 \\
0753135 \\
0753135 \\
0752309\end{array}$ & $\begin{array}{l}\text { S } \\
\text { F } \\
- \\
- \\
\text { S }\end{array}$ & $\begin{array}{l}\text { FSP Recreation } \\
\text { McReese } \\
\text { Allyn } \\
\text { Norwich Eaton Pharm. } \\
\text { S. New Berlin }\end{array}$ & $\begin{array}{c}1959 \\
-- \\
1965 \\
-- \\
--\end{array}$ & $\begin{array}{r}990 \\
995 \\
1003 \\
1010 \\
1055\end{array}$ & $\begin{array}{r}229 \\
418 \\
230 \\
375 \\
-\end{array}$ & $\begin{array}{r}229 \\
373 \\
230 \\
-- \\
--\end{array}$ & $\begin{array}{r}-- \\
373 \\
-- \\
-- \\
--\end{array}$ & $\begin{array}{l}\text { QRNR } \\
\text { BDRK } \\
\text { QRNR } \\
-- \\
--\end{array}$ \\
\hline $\begin{array}{l}137 \\
138 \\
140 \\
141\end{array}$ & $\begin{array}{l}423204 \\
423252 \\
423311 \\
423311\end{array}$ & $\begin{array}{l}0753125 \\
0753049 \\
0753117 \\
0753136\end{array}$ & $\begin{array}{l}- \\
\\
-\end{array}$ & $\begin{array}{l}\text { Nassar } \\
\text { Marango } \\
\text { Sheffield Chemical } \\
\text { Staefanelli Motel }\end{array}$ & $\begin{array}{l}1965 \\
1964 \\
1939 \\
1966\end{array}$ & $\begin{array}{l}1017 \\
1000 \\
1005 \\
1045\end{array}$ & $\begin{array}{r}460 \\
312 \\
310 \\
67\end{array}$ & $\begin{array}{c}-- \\
-- \\
-- \\
56\end{array}$ & $\begin{array}{r}33 \\
-- \\
303 \\
--\end{array}$ & $\begin{array}{l}\text { BDRK } \\
\text { QRNR } \\
\text { BDRK } \\
\text { QRNR }\end{array}$ \\
\hline 142 & 423314 & 0753116 & $\mathbf{S}$ & Sheffield Chemical & $06-01-44$ & 1005 & 284 & -- & 284 & BDRK \\
\hline $\begin{array}{l}143 \\
144 \\
145 \\
146 \\
147\end{array}$ & $\begin{array}{l}423320 \\
423321 \\
423325 \\
423327 \\
423328\end{array}$ & $\begin{array}{l}0753125 \\
0753117 \\
0753102 \\
0753130 \\
0754313\end{array}$ & $\begin{array}{l}\text { S } \\
- \\
- \\
\text { S }\end{array}$ & $\begin{array}{l}\text { Norwich Eaton Pharm. } \\
\text { Norwich Eaton Pharm. } \\
\text { Christ Science Church } \\
\text { Norwich Eaton Pharm. } \\
\text { Keator }\end{array}$ & $\begin{array}{c}1954 \\
1950 \\
-- \\
\ldots \\
1953\end{array}$ & $\begin{array}{l}1005 \\
1010 \\
1025 \\
1000 \\
1621\end{array}$ & $\begin{array}{r}130 \\
300 \\
346 \\
20 \\
35\end{array}$ & $\begin{array}{r}120 \\
300 \\
250 \\
-- \\
34\end{array}$ & $\begin{array}{r}-- \\
250 \\
-- \\
34\end{array}$ & $\begin{array}{l}\text { QRNR } \\
\text { QRNR } \\
\text { BDRK } \\
\text { QRNR } \\
\text { BDRK }\end{array}$ \\
\hline $\begin{array}{l}148 \\
149 \\
150 \\
151 \\
152\end{array}$ & $\begin{array}{l}423329 \\
423330 \\
423330 \\
423331 \\
423335\end{array}$ & $\begin{array}{l}0753130 \\
0752132 \\
0753125 \\
0753131 \\
0752125\end{array}$ & $\begin{array}{l}\text { S } \\
- \\
\text { S } \\
-\end{array}$ & $\begin{array}{l}\text { Norwich Eaton Pharm. } \\
\text { Collins } \\
\text { Norwich Eaton Pharm. } \\
\text { Norwich Eaton Pharm. } \\
\text { Friedel }\end{array}$ & $\begin{array}{c}1956 \\
1944 \\
-- \\
-- \\
1960\end{array}$ & $\begin{array}{l}1005 \\
1085 \\
1005 \\
1000 \\
1080\end{array}$ & $\begin{array}{l}130 \\
330 \\
330 \\
150 \\
300\end{array}$ & $\begin{array}{l}130 \\
330 \\
330 \\
150 \\
300\end{array}$ & $\begin{array}{r}-- \\
305 \\
-- \\
--\end{array}$ & $\begin{array}{l}\text { QRNR } \\
\text { QRNR } \\
\text { BDRK } \\
\text { QRNR } \\
\text { QRNR }\end{array}$ \\
\hline
\end{tabular}


Table 1.--Records of wells and test holes--continued.

[Dashes indicate no data. Locations shown on pls. 1A, 1B.]

Well
number $\frac{\text { Water level }}{\text { feet }} \begin{gathered}\text { Yield } \\ \text { date }\end{gathered}$ (gal/min) $\quad \begin{gathered}\text { Remarks } \\ \text { (Depths of geologic units given in feet below land surface) }\end{gathered}$

\begin{tabular}{|c|c|c|c|c|}
\hline 102 & - & -- & 60 &  \\
\hline 103 & 12 & 1958 & 60 & Sand \& gravel at surface, $70 \& 151-169$, silty sand at intervening intervals. \\
\hline 104 & -- & -- & 25 & Ends in gravel below silty sand. \\
\hline 105 & -- & -- & 20 & $\ldots$ \\
\hline 106 & 20 & $09-66$ & 35 & 0-50 fine sand; 50-95 stones; $95-97$ sand; $97-99$ boulders; $99-103$ gravel. \\
\hline 107 & 15 & $08-62$ & 12 & -- \\
\hline 108 & -- & -- & 10 & 0-39 HP w/SH; 39-44 SH. Location approximate. \\
\hline 109 & 15 & $09-66$ & 20 & Gravel, very fine sand. Water cloudy first few months. Well depth uncertain. \\
\hline 110 & -- & -- & 50 & $0-85$ gravel. \\
\hline 111 & -- & -- & 20 & -- \\
\hline 112 & - & -. & 30 & -- \\
\hline 113 & -- & -- & -- & -- \\
\hline 114 & -- & -- & 30 & -- \\
\hline 115 & - & - & -- & -- \\
\hline 116 & - & -- & 5 & -- \\
\hline 117 & -- & -- & 10 & 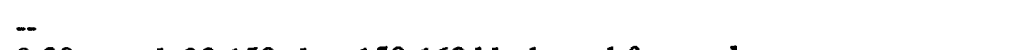 \\
\hline 118 & -- & -- & 25 & 0-30 gravel; 30-150 clay; $150-160$ black sand \& gravel. \\
\hline 119 & -- & -- & 7 & -- \\
\hline 120 & -. & - & 30 & -- \\
\hline 121 & -- & -- & 4 & Gravel at surface, then very fine sand. \\
\hline 122 & -- & - & 4 & -- \\
\hline 123 & -- & -- & -- & Sulfur odor. Abundant blue clay, ends in gravel. \\
\hline 124 & - & -- & 30 & -- \\
\hline 125 & 15 & 1946 & 10 & 0-85 gravel; 85-152 BR. \\
\hline 126 & -- & -- & -- & $0-40$ sand; $40-190 \mathrm{BR}$. \\
\hline 127 & -- & -- & -- & -- \\
\hline 128 & 46 & 1947 & 12 & 0-111 HP; 111-127 hard sandy rock. Location approximate. \\
\hline 129 & -- & -- & 8 & $-\infty$ \\
\hline 130 & -- & -- & 30 & Sulfur odor, formerly flowed frequently. \\
\hline 131 & -- & -- & 25 & Penetrates clay, ends in black sand \& gravel. Flows sometimes. \\
\hline 132 & -- & -- & 500 & 20-160 blue clay; $160-229$ gravel. Flows in Spring. \\
\hline 133 & -- & -- & 125 & $0-220$ clay. Location approximate. Water salty below $500 \mathrm{ft}$. \\
\hline 134 & -- & -- & - & $0-15$ gravel; $15-225$ clay?; $225-230$ black gravel. \\
\hline 135 & -- & - & 150 & Depth approximate. \\
\hline 136 & 16 & $10-65$ & -- & -- \\
\hline 137 & -- & -- & 7 & -- \\
\hline 138 & 10 & $07-64$ & 30 & 0-199 clay; $199-305$ sand; 305-312 gravel. \\
\hline 140 & -- & -- & 220 & 0-303 clay; 303-310 BR. Water enters at $309 \mathrm{ft}$. \\
\hline 141 & 23 & $05-66$ & 30 & $\begin{array}{l}\text { 0-42 sand, gravel, \& HP; } 42-62 \text { sand \& gravel; } 62-67 \text { HP. Yields } 20-30 \text { GPM } \\
\text { from } 54-62 \mathrm{ft} \text {. }\end{array}$ \\
\hline 142 & 12 & $06-44$ & 200 & $0-276$ clay; $276-284$ sand, gravel, some clay. \\
\hline 143 & -- & - & 150 & 0-30 gravel; $30-120$ clay; $120-130$ gravel. \\
\hline 144 & - & - & 150 & 0-25 gravel; $25-280$ clay, some gravel; $280-300$ gravel. \\
\hline 145 & - & -- & - & $0-250$ cement clay; $250-346 \mathrm{BR}$. \\
\hline 146 & -- & - & 150 & Dug 10 foot pit for tank, water entered hole. \\
\hline 147 & 15 & 1953 & -- & 0-34 SH and HP; 34-35 SH. Location approximate. \\
\hline 148 & -- & -- & 150 & -. \\
\hline 149 & 7 & 1944 & 10 & 0-300 clay; $300-330$ black gravel. \\
\hline 150 & -- & -- & -- & 0-20 gravel; $20-305$ clay; $305-330$ BR. Little or no water, abandoned. \\
\hline 151 & -- & -- & - & $0-20$ gravel; $20-130$ clay; $130-150$ fine gravel \& sand. Small yield, not used. \\
\hline 152 & -- & -- & -- & -- \\
\hline
\end{tabular}


Table 1.--Records of wells and test holes--continued.

[Dashes indicate no data. Locations shown on pls. 1A, 1B.]

\begin{tabular}{|c|c|c|c|c|c|c|c|c|c|c|}
\hline \multirow[b]{2}{*}{$\begin{array}{l}\text { Well } \\
\text { number }\end{array}$} & \multicolumn{3}{|c|}{ Location } & \multirow[b]{2}{*}{ Owner } & \multirow[b]{2}{*}{$\begin{array}{c}\text { Date } \\
\text { drilled }\end{array}$} & \multirow{2}{*}{$\begin{array}{c}\text { Altitude } \\
\text { of land } \\
\text { surface } \\
\text { (feet) }\end{array}$} & \multirow[b]{2}{*}{$\begin{array}{l}\text { Well } \\
\text { depth } \\
\text { (feet) }\end{array}$} & \multirow[b]{2}{*}{$\begin{array}{l}\text { Casing } \\
\text { depth } \\
\text { (feet) }\end{array}$} & \multirow{2}{*}{$\begin{array}{l}\text { Depth } \\
\text { to } \\
\text { bedrock } \\
\text { (feet) }\end{array}$} & \multirow[b]{2}{*}{$\begin{array}{c}\text { Geologic } \\
\text { unit }\end{array}$} \\
\hline & $\begin{array}{l}\text { Lati- } \\
\text { tude } \\
\ldots, n\end{array}$ & $\begin{array}{c}\text { Longi- } \\
\text { tude } \\
\cdots,\end{array}$ & $\begin{array}{l}\text { Accu- } \\
\text { racy }\end{array}$ & & & & & & & \\
\hline $\begin{array}{l}153 \\
154 \\
155 \\
156 \\
157\end{array}$ & $\begin{array}{l}423340 \\
423344 \\
423348 \\
423350 \\
423352\end{array}$ & $\begin{array}{l}0753116 \\
0752118 \\
0753116 \\
0752102 \\
0752037\end{array}$ & $\begin{array}{l}S \\
- \\
- \\
-\end{array}$ & $\begin{array}{l}\text { Camp } \\
\text { Cuddihy } \\
\text { Martin } \\
\text { Pike }\end{array}$ & $\begin{array}{c}-- \\
-- \\
- \\
1965 \\
1960\end{array}$ & $\begin{array}{l}1017 \\
1070 \\
1017 \\
1070 \\
1065\end{array}$ & $\begin{array}{r}160 \\
340 \\
93 \\
285 \\
280\end{array}$ & $\begin{array}{r}340 \\
-\overline{-} \\
285 \\
280\end{array}$ & $\begin{array}{l}-- \\
-- \\
-- \\
--\end{array}$ & $\begin{array}{l}\text { QRNR } \\
\text { QRNR } \\
\text { QRNR } \\
\text { QRNR } \\
\text { QRNR }\end{array}$ \\
\hline $\begin{array}{l}159 \\
160 \\
161 \\
162 \\
163\end{array}$ & $\begin{array}{l}423404 \\
423407 \\
423412 \\
423437 \\
423447\end{array}$ & $\begin{array}{l}0753138 \\
0753154 \\
0752025 \\
0752001 \\
0755151\end{array}$ & $\begin{array}{l}\text { S } \\
\text { S } \\
- \\
- \\
\text { F }\end{array}$ & $\begin{array}{l}\text { Midstate Aviation } \\
\text { Wilson } \\
\text { Camp } \\
\text { Vanwarner } \\
\text { Holtmart }\end{array}$ & $\begin{array}{c}1945 \\
-- \\
1960 \\
-- \\
1952\end{array}$ & $\begin{array}{l}1020 \\
1100 \\
1072 \\
1070 \\
1160\end{array}$ & $\begin{array}{r}200 \\
316 \\
160 \\
70 \\
51\end{array}$ & $\begin{array}{r}200 \\
98 \\
160 \\
70 \\
51\end{array}$ & $\begin{array}{l}-- \\
98 \\
-- \\
-- \\
--\end{array}$ & $\begin{array}{l}\text { QRNR } \\
\text { BDRK } \\
\text { QRNR } \\
\text { SDGV } \\
\text { GRVL }\end{array}$ \\
\hline $\begin{array}{l}164 \\
165 \\
166 \\
167 \\
168\end{array}$ & $\begin{array}{l}423447 \\
423447 \\
423448 \\
423449 \\
423453\end{array}$ & $\begin{array}{l}0755153 \\
0755208 \\
0755153 \\
0755154 \\
0755149\end{array}$ & $\begin{array}{l}F \\
F \\
F \\
F \\
F\end{array}$ & $\begin{array}{l}\text { Jinks } \\
\text { Hakes } \\
\text { Hull } \\
\text { Potter } \\
\text { Livermore }\end{array}$ & $\begin{array}{l}1947 \\
1945 \\
1940 \\
1940 \\
1946\end{array}$ & $\begin{array}{l}1150 \\
1099 \\
1146 \\
1144 \\
1135\end{array}$ & $\begin{array}{r}112 \\
60 \\
100 \\
51 \\
80\end{array}$ & $\begin{array}{l}90 \\
60 \\
80 \\
51 \\
75\end{array}$ & $\begin{array}{l}90 \\
-\overline{80} \\
\overline{75}\end{array}$ & $\begin{array}{l}\text { BDRK } \\
\text { GRVL } \\
\text { BDRK } \\
\text { GRVL } \\
\text { BDRK }\end{array}$ \\
\hline $\begin{array}{l}169 \\
170 \\
171 \\
172 \\
173\end{array}$ & $\begin{array}{l}423507 \\
423511 \\
423533 \\
423542 \\
423545\end{array}$ & $\begin{array}{l}0752005 \\
0755105 \\
0755038 \\
0752020 \\
0752018\end{array}$ & $\begin{array}{l}- \\
\text { F } \\
\text { F } \\
- \\
-\end{array}$ & $\begin{array}{l}\text { Iannello } \\
\text { Hakes } \\
\text { Davis } \\
\text { Santore } \\
\text { Anson }\end{array}$ & $\begin{array}{c}-- \\
1947 \\
1954 \\
1963 \\
1965\end{array}$ & $\begin{array}{l}1090 \\
1178 \\
1137 \\
1130 \\
1115\end{array}$ & $\begin{array}{r}100 \\
108 \\
72 \\
57 \\
205\end{array}$ & $\begin{array}{r}100 \\
100 \\
72 \\
57 \\
190\end{array}$ & $\begin{array}{r}-- \\
100 \\
-- \\
--\end{array}$ & $\begin{array}{l}\text { QRNR } \\
\text { BDRK } \\
\text { QRNR } \\
\text { QRNR } \\
\text { BDRK }\end{array}$ \\
\hline $\begin{array}{l}174 \\
175 \\
176 \\
177 \\
178\end{array}$ & $\begin{array}{l}423547 \\
423602 \\
423604 \\
423605 \\
423631\end{array}$ & $\begin{array}{l}\mathbf{0 7 5 2 0 1 8} \\
\mathbf{0 7 5 5 0 0 7} \\
0755009 \\
\mathbf{0 7 5 2 0 2 9} \\
0755204\end{array}$ & $\begin{array}{l}\bar{F} \\
\text { F } \\
\bar{F}\end{array}$ & $\begin{array}{l}\text { Anson } \\
\text { Barrows } \\
\text { Hagen } \\
\text { Dunham } \\
\text { Eaton }\end{array}$ & $\begin{array}{c}1961 \\
-- \\
-- \\
1956 \\
--\end{array}$ & $\begin{array}{l}1110 \\
1169 \\
1160 \\
1140 \\
1450\end{array}$ & $\begin{array}{l}205 \\
118 \\
165 \\
278 \\
110\end{array}$ & $\begin{array}{r}190 \\
70 \\
103 \\
278 \\
45\end{array}$ & $\begin{array}{r}190 \\
70 \\
103 \\
- \\
45\end{array}$ & $\begin{array}{l}\text { BDRK } \\
\text { BDRK } \\
\text { BDRK } \\
-- \\
\text { BDRK }\end{array}$ \\
\hline 179 & 423650 & 0753152 & $S$ & Scheuerman & 1976 & 1025 & 12 & 10 & -- & OTSH \\
\hline $\begin{array}{l}180 \\
181 \\
182 \\
183\end{array}$ & $\begin{array}{l}423656 \\
423658 \\
423700 \\
423700\end{array}$ & $\begin{array}{l}0753143 \\
0753143 \\
0753137 \\
0753138\end{array}$ & $\begin{array}{l}S \\
S \\
S \\
S\end{array}$ & $\begin{array}{l}\text { Kellogg } \\
\text { Frank } \\
\text { Ebert } \\
\text { Whaley }\end{array}$ & $\begin{array}{l}1961 \\
1950 \\
1945 \\
1900\end{array}$ & $\begin{array}{l}1030 \\
1031 \\
1034 \\
1034\end{array}$ & $\begin{array}{l}14 \\
16 \\
12 \\
20\end{array}$ & $\begin{array}{l}12 \\
14 \\
10 \\
18\end{array}$ & $\begin{array}{l}-- \\
-- \\
-- \\
-\end{array}$ & $\begin{array}{l}\text { OTSH } \\
\text { OTSH } \\
\text { OTSH } \\
\text { OTSH }\end{array}$ \\
\hline $\begin{array}{l}184 \\
185 \\
186 \\
187 \\
188\end{array}$ & $\begin{array}{l}423703 \\
423704 \\
423704 \\
423704 \\
423705\end{array}$ & $\begin{array}{l}\mathbf{0 7 5 3 1 5 0} \\
\mathbf{0 7 5 3 1 3 2} \\
0753144 \\
0753152 \\
0753134\end{array}$ & $\begin{array}{l}S \\
S \\
S \\
S \\
S\end{array}$ & $\begin{array}{l}\text { Smith } \\
\text { Wright } \\
\text { Pike } \\
\text { Jones } \\
\text { Labor }\end{array}$ & $\begin{array}{l}1969 \\
1977 \\
1967 \\
1979 \\
1960\end{array}$ & $\begin{array}{l}1034 \\
1035 \\
1038 \\
1033 \\
1038\end{array}$ & $\begin{array}{r}12 \\
334 \\
18 \\
16 \\
21\end{array}$ & $\begin{array}{r}10 \\
334 \\
16 \\
14 \\
19\end{array}$ & $\begin{array}{l}- \\
- \\
- \\
- \\
-\end{array}$ & $\begin{array}{l}\text { OTSH } \\
\text { KMTC } \\
\text { OTSH } \\
\text { OTSH } \\
\text { OTSH }\end{array}$ \\
\hline $\begin{array}{l}189 \\
190 \\
191 \\
192 \\
193\end{array}$ & $\begin{array}{l}423705 \\
423707 \\
423708 \\
423708 \\
423711\end{array}$ & $\begin{array}{l}0753616 \\
0753209 \\
0753115 \\
0754930 \\
0753138\end{array}$ & $\begin{array}{l}\text { F } \\
\text { S } \\
\text { S } \\
\text { F } \\
\text { S }\end{array}$ & $\begin{array}{l}\text { Park } \\
\text { Augustyn } \\
\text { Stanton } \\
\text { Burrows } \\
\text { Crandall }\end{array}$ & $\begin{array}{c}1946 \\
1970 \\
1948 \\
-\ddot{-} \\
1979\end{array}$ & $\begin{array}{l}1280 \\
1065 \\
1038 \\
1177 \\
1045\end{array}$ & $\begin{array}{r}91 \\
210 \\
308 \\
100 \\
202\end{array}$ & $\begin{array}{r}91 \\
210 \\
300 \\
100 \\
202\end{array}$ & $\begin{array}{r}-- \\
300 \\
-- \\
--\end{array}$ & $\begin{array}{l}\text { QRNR } \\
\text { KMTC } \\
\text { BDRK } \\
\text { GRVL } \\
\text { KMTC }\end{array}$ \\
\hline $\begin{array}{l}194 \\
195 \\
196 \\
197 \\
198\end{array}$ & $\begin{array}{l}423711 \\
423714 \\
423714 \\
423722 \\
423725\end{array}$ & $\begin{array}{l}0753203 \\
0753142 \\
0754925 \\
0753205 \\
0753227\end{array}$ & $\begin{array}{l}\text { S } \\
\text { S } \\
\text { F } \\
S \\
S\end{array}$ & $\begin{array}{l}\text { N. Norwich } \\
\text { Moon } \\
\text { Maxson } \\
\text { USGS } \\
\text { Brown }\end{array}$ & $\begin{array}{c}1950 \\
1950 \\
1954 \\
09-18-80 \\
1971\end{array}$ & $\begin{array}{l}1035 \\
1035 \\
1175 \\
1050 \\
1110\end{array}$ & $\begin{array}{r}85 \\
219 \\
73 \\
86 \\
151\end{array}$ & $\begin{array}{r}85 \\
200 \\
73 \\
-- \\
100\end{array}$ & $\begin{array}{r}200 \\
-\overline{8} \\
86 \\
100\end{array}$ & $\begin{array}{l}\text { KMTC } \\
\text { BDRK } \\
\text { GRVL } \\
\text { BDRK } \\
\text { BDRK }\end{array}$ \\
\hline $\begin{array}{l}199 \\
200 \\
201 \\
202 \\
203\end{array}$ & $\begin{array}{l}423726 \\
423729 \\
423733 \\
423738 \\
423739\end{array}$ & $\begin{array}{l}0753228 \\
0753228 \\
0753211 \\
0753210 \\
0753209\end{array}$ & $\begin{array}{l}S \\
S \\
S \\
S \\
S\end{array}$ & $\begin{array}{l}\text { Van Steinburg } \\
\text { Reese } \\
\text { USGS } \\
\text { Bates } \\
\text { Eggleston }\end{array}$ & $\begin{array}{c}1955 \\
1972 \\
09-19-80 \\
1979 \\
1950\end{array}$ & $\begin{array}{l}1110 \\
1105 \\
1050 \\
1030 \\
1045\end{array}$ & $\begin{array}{r}192 \\
201 \\
83 \\
130 \\
20\end{array}$ & $\begin{array}{r}-- \\
60 \\
-- \\
130 \\
18\end{array}$ & $\begin{array}{l}-- \\
60 \\
-- \\
-- \\
--\end{array}$ & $\begin{array}{l}\text { BDRK } \\
\text { BDRK } \\
\text { SDGV } \\
\text { LAKE } \\
\text { OTSH }\end{array}$ \\
\hline
\end{tabular}


Table 1.--Records of wells and test holes--continued.

[Dashes indicate no data. Locations shown on pls. 1A, 1B.]

\begin{tabular}{|c|c|c|c|c|}
\hline \multirow{2}{*}{$\begin{array}{l}\text { Well } \\
\text { number }\end{array}$} & \multicolumn{2}{|c|}{ Water level } & \multirow{2}{*}{$\begin{array}{c}\text { Yield } \\
(\mathrm{gal} / \mathrm{min})\end{array}$} & \multirow{2}{*}{$\begin{array}{l}\text { Remarks } \\
\text { (Depths of geologic units given in feet below land surface) }\end{array}$} \\
\hline & feet & date & & \\
\hline 153 & - & - & 25 & Penetrates sand \& gravel, then clay, ends in coarse gravel. \\
\hline 154 & $\cdots$ & -- & 15 & - \\
\hline 155 & -- & -. & 20 & -- \\
\hline 156 & 10 & 1965 & 25 & 0-12 gravel; $12-18$ boulder; $18-270$ clay; $270-285$ sand. \\
\hline 157 & 15 & 1960 & 20 & Ends in black sand. \\
\hline 159 & $\ldots$ & - & 15 & -. \\
\hline 160 & -- & - & $\cdots$ & Sulfur odor. \\
\hline 161 & -- & -- & 15 & - \\
\hline 162 & -- & -- & -. & 0-50 gravel; 50-70 black sand. Test shows excessive iron. \\
\hline 163 & 1 & $09-52$ & 18 & $0-51$ gravel. \\
\hline 164 & -- & $07-47$ & 2 & 0-90 gravel; 90-112 BR. Flowed 2 GPM in 1947, still flowing in 1970. \\
\hline 165 & 5 & $09-45$ & 20 & $0-60$ dirty gravel. Some water at $12 \mathrm{ft}$. \\
\hline 166 & -- & - & 2 & $0-32$ clean gravel; $32-80$ fine sand; $80-100$ BR. \\
\hline 167 & -- & -- & - & $0-51$ clean gravel. \\
\hline 168 & 38 & $09-46$ & 20 & 0-75 dirty gravel; $75-80 \mathrm{BR}$. \\
\hline 169 & -- & -- & 10 & Sulfur odor. \\
\hline 170 & - & -. & 20 & $0-100$ dirty gravel; $100-108 \mathrm{BR}$ \\
\hline 171 & -- & - & 30 & 0-16 gravel; $16-72$ clay; 72 fine gravel. \\
\hline 172 & - & -. & 18 & $0-10$ clay; $10-57$ black sand \& gravel. Overflows frequently. \\
\hline 173 & -- & -- & 35 & 0-190 gravel, boulders; $190-205 \mathrm{BR}$. \\
\hline 174 & 30 & 1961 & 12 & 0-190 gravel; 190-205 BR. \\
\hline 175 & -- & -- & 10 & $0-70$ sand \& gravel. \\
\hline 176 & -- & -- & 3 & 0-30 gravel; 30-103 blue clay; $103-165 \mathrm{BR}$. \\
\hline 177 & -- & - & 15 & \\
\hline 178 & -- & - & 14 & 0-45 HP, SH fragments; 45-110 BR. Location approximate. \\
\hline 179 & 10 & 1976 & -- & $\begin{array}{l}\text { Hard water. Owner reports very fine sand (quicksand) from } 18-20 \mathrm{ft} \text {. Pulled back } \\
\text { to } 12 \mathrm{ft} \text {. }\end{array}$ \\
\hline 180 & 9 & $06-61$ & -- & Well in basement, supply adequate. \\
\hline 181 & 4 & 1950 & -- & Owner reports $0-7$ fine sand \& gravel. Well in basement, hard water. \\
\hline 182 & 9 & -- & -- & Owner reports $0-8$ sand, then coarse gravel. Hard water, calcium deposits. \\
\hline 183 & -- & -- & -- & Lab analysis $2 / 75$; Nitrate reported as nitrogen: $4.2 \mathrm{MG} / \mathrm{L}$, chloride: $17 \mathrm{MG} / \mathrm{L}$. \\
\hline 184 & 3 & 1969 & -- & Owner reports $0-12$ sand; 12 clay. Suction pump. Hard water. \\
\hline 185 & 8 & 1977 & 30 & Supplies adult home. Hydrogen sulfide odor. \\
\hline 186 & 12 & - & - & Hard water. Owner reports well has never failed. \\
\hline 187 & 8 & 1979 & - & 0-16 sand, fine gravel. Suction pump. Water has been tested, fairly soft. \\
\hline 188 & 14 & - & -- & Hard water. Owner replaces well point annually due to plugging. \\
\hline 189 & -- & $09-46$ & 10 & 0-91 HP \& SH. \\
\hline 190 & -- & -- & - & Slightly hard water. Hydrogen sulfide odor and taste. \\
\hline 191 & 20 & -- & -- & 0-300 HP; $300-308$ BR. Hard water. Supplies 3 families \& 150 Cattle. \\
\hline 192 & -- & - & 30 & $0-100$ gravel. \\
\hline 193 & 14 & $06-03-80$ & -- & Abandoned, poor yield. Silty sand. Meas. point is $1.6 \mathrm{ft}$ above LSD. \\
\hline 194 & 8 & $06-05-80$ & -- & Poor yield, not used for drinking. Meas. point is $1.8 \mathrm{ft}$ above LSD. \\
\hline 195 & -- & -- & -- & Well supplies 2 homes. Slight hydrogen sulfide odor. \\
\hline 196 & 17 & 03-54 & 30 & $0-73$ dirty gravel. \\
\hline 197 & 23 & $09-18-80$ & -- & 0-52 sand \& gravel; $52-56$ clayey silt; $56-81$ sand \& gravel; $81-86$ till; $86 \mathrm{SH}$. \\
\hline 198 & -- & -- & 10 & Depth to bedrock questionable, based on nearby data. \\
\hline 199 & 40 & -- & -- & Hard water, slight hydrogen sulfide taste. Depth to rock unknown. \\
\hline 200 & -- & -- & 13 & Hard water, hydrogen sulfide odor. Gravel, clay, ends in $10 \mathrm{ft}$ gravel. \\
\hline 201 & 20 & $09-19-80$ & $\ldots$ & 0-16 sand \& gravel; $16-31$ sandy silt; $31-37$ sand \& gravel; $37-83$ lacust. sed. \\
\hline 202 & 2 & $05-29-80$ & -- & Abandoned, poor yield. Casing at LSD. Penetrates lacust. sed. \\
\hline 203 & -- & -- & -- & Suction pump. Hard water. Adequate supply. \\
\hline
\end{tabular}


Table 1.--Records of wells and test holes--continued.

[Dashes indicate no data. Locations shown on pls. 1A, 1B.]

\begin{tabular}{|c|c|c|c|c|c|c|c|c|c|c|}
\hline \multirow[b]{2}{*}{$\begin{array}{c}\text { Well } \\
\text { number }\end{array}$} & \multicolumn{3}{|c|}{ Location } & \multirow[b]{2}{*}{ Owner } & \multirow[b]{2}{*}{$\begin{array}{l}\text { Date } \\
\text { drilled }\end{array}$} & \multirow{2}{*}{$\begin{array}{l}\text { Altitude } \\
\text { of land } \\
\text { surface } \\
\text { (feet) }\end{array}$} & \multirow[b]{2}{*}{$\begin{array}{l}\text { Well } \\
\text { depth } \\
\text { (feet) }\end{array}$} & \multirow[b]{2}{*}{$\begin{array}{l}\text { Casing } \\
\text { depth } \\
\text { (feet) }\end{array}$} & \multirow{2}{*}{$\begin{array}{c}\text { Depth } \\
\text { to } \\
\text { bedrock } \\
\text { (feet) }\end{array}$} & \multirow[b]{2}{*}{$\begin{array}{c}\text { Geologic } \\
\text { unit }\end{array}$} \\
\hline & $\begin{array}{l}\text { Lati- } \\
\text { tude } \\
\ldots .\end{array}$ & $\begin{array}{c}\begin{array}{c}\text { Longi- } \\
\text { tude }\end{array} \\
\ldots .\end{array}$ & $\begin{array}{l}\text { Accu- } \\
\text { racy }\end{array}$ & & & & & & & \\
\hline $\begin{array}{l}204 \\
205 \\
206 \\
207 \\
209\end{array}$ & $\begin{array}{l}423740 \\
423741 \\
423742 \\
423753 \\
423802\end{array}$ & $\begin{array}{l}0753156 \\
0753156 \\
0753149 \\
0754742 \\
0753246\end{array}$ & $\begin{array}{l}S \\
S \\
S \\
F \\
S\end{array}$ & $\begin{array}{l}\text { Meyers } \\
\text { Decker } \\
\text { Seibert } \\
\text { Loomis } \\
\text { Homovich }\end{array}$ & $\begin{array}{c}08-01-74 \\
05-01-77 \\
1967 \\
1950 \\
1958\end{array}$ & $\begin{array}{l}1065 \\
1070 \\
1105 \\
1210 \\
1105\end{array}$ & $\begin{array}{r}65 \\
66 \\
119 \\
44 \\
161\end{array}$ & $\begin{array}{r}65 \\
66 \\
119 \\
44 \\
85\end{array}$ & $\begin{array}{l}- \\
\cdots \\
-- \\
85\end{array}$ & $\begin{array}{l}\text { KMTC } \\
\text { KMTC } \\
\text { KMTC } \\
\text { QRNR } \\
\text { BDRK }\end{array}$ \\
\hline $\begin{array}{l}210 \\
211 \\
212 \\
213 \\
214\end{array}$ & $\begin{array}{l}423814 \\
423829 \\
423830 \\
423830 \\
423832\end{array}$ & $\begin{array}{l}0753148 \\
0753249 \\
0751949 \\
0753253 \\
0753252\end{array}$ & $\begin{array}{l}\mathbf{S} \\
\mathbf{S} \\
\overline{\mathbf{S}} \\
\mathbf{S}\end{array}$ & $\begin{array}{l}\text { Pike } \\
\text { Tarbell } \\
\text { Mobil Station } \\
\text { Landon } \\
\text { Webster }\end{array}$ & $\begin{array}{l}1940 \\
1975 \\
1963 \\
1978 \\
1975\end{array}$ & $\begin{array}{l}1125 \\
1060 \\
1110 \\
1070 \\
1055\end{array}$ & $\begin{array}{l}90 \\
13 \\
86 \\
30 \\
12\end{array}$ & $\begin{array}{l}90 \\
11 \\
86 \\
28 \\
10\end{array}$ & $\begin{array}{l}- \\
- \\
-- \\
-- \\
-\end{array}$ & $\begin{array}{l}\text { QRNR } \\
\text { OTSH } \\
\text { QRNR } \\
\text { OTSH } \\
\text { OTSH }\end{array}$ \\
\hline $\begin{array}{l}215 \\
216 \\
217 \\
218 \\
219\end{array}$ & $\begin{array}{l}423834 \\
423835 \\
423842 \\
423845 \\
423849\end{array}$ & $\begin{array}{l}0751947 \\
0753215 \\
0751948 \\
0754656 \\
0751953\end{array}$ & $\begin{array}{l}\bar{s} \\
\text { S } \\
\bar{F} \\
-\end{array}$ & $\begin{array}{l}\text { Sickler } \\
\text { USGS } \\
\text { Currie } \\
\text { Morse } \\
\text { Nelson }\end{array}$ & $\begin{array}{r}1965 \\
01-81 \\
1955 \\
1954 \\
1965\end{array}$ & $\begin{array}{l}1110 \\
1070 \\
1115 \\
1240 \\
1145\end{array}$ & $\begin{array}{r}110 \\
104 \\
90 \\
86 \\
140\end{array}$ & $\begin{array}{r}110 \\
90 \\
82 \\
140\end{array}$ & $\begin{array}{c}-- \\
\ddot{-} \\
82 \\
--\end{array}$ & $\begin{array}{l}\text { QRNR } \\
\text { KMTC } \\
\text { GRVL } \\
\text { BDRK } \\
\text { GRVL }\end{array}$ \\
\hline $\begin{array}{l}220 \\
221 \\
222 \\
223 \\
224\end{array}$ & $\begin{array}{l}421153 \\
423849 \\
423850 \\
423851 \\
423851\end{array}$ & $\begin{array}{l}0753536 \\
0753247 \\
0754653 \\
0753249 \\
0754706\end{array}$ & $\begin{array}{l}- \\
\text { S } \\
\text { F } \\
\text { S } \\
\text { S }\end{array}$ & $\begin{array}{l}\text { Vandusen } \\
\text { Bullock } \\
\text { Baldwin } \\
\text { Irwin } \\
\text { S. Otselic Village }\end{array}$ & $\begin{array}{l}1946 \\
1952 \\
1951 \\
1950 \\
1959\end{array}$ & $\begin{array}{r}960 \\
1080 \\
1222 \\
1079 \\
1205\end{array}$ & $\begin{array}{r}105 \\
39 \\
70 \\
25 \\
95\end{array}$ & $\begin{array}{r}105 \\
39 \\
70 \\
23 \\
85\end{array}$ & $\begin{array}{l}-- \\
-- \\
-- \\
--\end{array}$ & $\begin{array}{l}\text { QRNR } \\
\text { KMTC } \\
\text { GRVL } \\
\text { KMTC } \\
\text { QRNR }\end{array}$ \\
\hline $\begin{array}{l}225 \\
226 \\
227 \\
228 \\
229\end{array}$ & $\begin{array}{l}423852 \\
423852 \\
423853 \\
423853 \\
423854\end{array}$ & $\begin{array}{l}0753236 \\
0753246 \\
0753307 \\
0753312 \\
0753240\end{array}$ & $\begin{array}{l}S \\
S \\
S \\
S \\
S\end{array}$ & $\begin{array}{l}\text { Law } \\
\text { Cook } \\
\text { Fairbanks } \\
\text { Treschow } \\
\text { Onyan }\end{array}$ & $\begin{array}{c}1971 \\
1939 \\
09-07-63 \\
1961 \\
1979\end{array}$ & $\begin{array}{l}1090 \\
1081 \\
1115 \\
1135 \\
1098\end{array}$ & $\begin{array}{r}62 \\
18 \\
72 \\
123 \\
80\end{array}$ & $\begin{array}{l}62 \\
16 \\
69 \\
60 \\
75\end{array}$ & $\begin{array}{l}-- \\
-- \\
69 \\
60 \\
75\end{array}$ & $\begin{array}{l}\text { KMTC } \\
\text { KMTC } \\
\text { BDRK } \\
\text { BDRK } \\
\text { BDRK }\end{array}$ \\
\hline $\begin{array}{l}230 \\
231 \\
232 \\
233 \\
234\end{array}$ & $\begin{array}{l}423854 \\
423855 \\
423856 \\
423856 \\
423857\end{array}$ & $\begin{array}{l}0754709 \\
0753236 \\
0753203 \\
0753219 \\
0754711\end{array}$ & $\begin{array}{l}\mathrm{F} \\
\mathrm{S} \\
\mathrm{S} \\
\mathrm{S} \\
\mathrm{F}\end{array}$ & $\begin{array}{l}\text { Martin } \\
\text { Law } \\
\text { Rabig } \\
\text { Marks } \\
\text { Halburt }\end{array}$ & $\begin{array}{c}1955 \\
1945 \\
10-01-77 \\
1966 \\
1953\end{array}$ & $\begin{array}{l}1216 \\
1100 \\
1108 \\
1150 \\
1217\end{array}$ & $\begin{array}{r}75 \\
161 \\
125 \\
30 \\
40\end{array}$ & $\begin{array}{r}75 \\
-- \\
58 \\
30 \\
40\end{array}$ & $\begin{array}{c}-- \\
58 \\
-- \\
--\end{array}$ & $\begin{array}{l}\text { SDGV } \\
\text { BDRK } \\
\text { BDRK } \\
\text { KMTC } \\
\text { SDGV }\end{array}$ \\
\hline $\begin{array}{l}235 \\
236 \\
237 \\
238 \\
239\end{array}$ & $\begin{array}{l}423858 \\
423900 \\
423901 \\
423901 \\
423904\end{array}$ & $\begin{array}{l}0753318 \\
0753247 \\
0753158 \\
0753315 \\
0753317\end{array}$ & $\begin{array}{l}S \\
S \\
S \\
S \\
S\end{array}$ & $\begin{array}{l}\text { Parry } \\
\text { Losee } \\
\text { Boise } \\
\text { Lewis } \\
\text { Parry }\end{array}$ & $\begin{array}{l}1968 \\
1972 \\
1974 \\
1960 \\
1950\end{array}$ & $\begin{array}{l}1115 \\
1110 \\
1110 \\
1120 \\
1130\end{array}$ & $\begin{array}{r}60 \\
147 \\
105 \\
23 \\
60\end{array}$ & $\begin{array}{l}40 \\
30 \\
58 \\
23 \\
60\end{array}$ & $\begin{array}{c}40 \\
30 \\
58 \\
-- \\
--\end{array}$ & $\begin{array}{l}\text { BDRK } \\
\text { BDRK } \\
\text { BDRK } \\
\text { OTSH } \\
\text { OTSH }\end{array}$ \\
\hline $\begin{array}{l}240 \\
241 \\
242 \\
243 \\
244\end{array}$ & $\begin{array}{l}423907 \\
423908 \\
423913 \\
423914 \\
423921\end{array}$ & $\begin{array}{l}0753320 \\
0753323 \\
0753323 \\
0753321 \\
0753330\end{array}$ & $\begin{array}{l}S \\
S \\
S \\
S \\
S\end{array}$ & $\begin{array}{l}\text { Brooks } \\
\text { Maynard } \\
\text { Alderman } \\
\text { USGS } \\
\text { Blanchard }\end{array}$ & $\begin{array}{c}1968 \\
1974 \\
1980 \\
09-17-80 \\
1950\end{array}$ & $\begin{array}{l}1130 \\
1140 \\
1132 \\
1133 \\
1150\end{array}$ & $\begin{array}{l}52 \\
38 \\
23 \\
31 \\
55\end{array}$ & $\begin{array}{l}52 \\
38 \\
21 \\
29 \\
55\end{array}$ & $\begin{array}{l}-- \\
-- \\
29 \\
--\end{array}$ & $\begin{array}{l}\text { OTSH } \\
\text { KMTC } \\
\text { OTSH } \\
\text { BDRK } \\
\text { OTSH }\end{array}$ \\
\hline $\begin{array}{l}245 \\
246\end{array}$ & $\begin{array}{l}423923 \\
423925\end{array}$ & $\begin{array}{l}0753044 \\
0753059\end{array}$ & $\begin{array}{l}S \\
S\end{array}$ & $\begin{array}{l}\text { Foster } \\
\text { USGS }\end{array}$ & $\begin{array}{c}01-74 \\
11-20-80\end{array}$ & $\begin{array}{l}1125 \\
1065\end{array}$ & $\begin{array}{l}210 \\
137\end{array}$ & $\begin{array}{r}90 \\
133\end{array}$ & $\begin{array}{r}90 \\
133\end{array}$ & $\begin{array}{l}\text { BDRK } \\
\text { BDRK }\end{array}$ \\
\hline $\begin{array}{l}247 \\
248 \\
249\end{array}$ & $\begin{array}{l}423925 \\
423926 \\
423927\end{array}$ & $\begin{array}{l}0753136 \\
0753406 \\
0753020\end{array}$ & $\begin{array}{l}F \\
S \\
S\end{array}$ & $\begin{array}{l}\text { Palmiter } \\
\text { Graham } \\
\text { Davenport }\end{array}$ & $\begin{array}{l}1965 \\
1973 \\
1975\end{array}$ & $\begin{array}{l}1150 \\
1295 \\
1205\end{array}$ & $\begin{array}{l}229 \\
170 \\
150\end{array}$ & $\begin{array}{r}21 \\
30 \\
150\end{array}$ & $\begin{array}{c}21 \\
30 \\
--\end{array}$ & $\begin{array}{l}\text { BDRK } \\
\text { BDRK } \\
\text { BDRK }\end{array}$ \\
\hline $\begin{array}{l}250 \\
251 \\
252 \\
253 \\
254\end{array}$ & $\begin{array}{l}423931 \\
423937 \\
423943 \\
423956 \\
424001\end{array}$ & $\begin{array}{l}0751957 \\
0753356 \\
0753353 \\
0753350 \\
0753352\end{array}$ & $\begin{array}{l}- \\
S \\
S \\
S \\
S\end{array}$ & $\begin{array}{l}\text { Padgett } \\
\text { Lloyd } \\
\text { Nelson } \\
\text { Stevens } \\
\text { Monroe }\end{array}$ & $\begin{array}{l}1906 \\
1956 \\
1978 \\
1950 \\
1967\end{array}$ & $\begin{array}{l}1145 \\
1230 \\
1250 \\
1170 \\
1175\end{array}$ & $\begin{array}{r}185 \\
97 \\
135 \\
32 \\
110\end{array}$ & $\begin{array}{r}185 \\
44 \\
112 \\
-- \\
28\end{array}$ & $\begin{array}{r}-- \\
44 \\
111 \\
\ddot{28}\end{array}$ & $\begin{array}{l}\text { QRNR } \\
\text { BDRK } \\
\text { BDRK } \\
\text { KMTC } \\
\text { BDRK }\end{array}$ \\
\hline
\end{tabular}


Table 1.--Records of wells and test holes--continued.

[Dashes indicate no data. Locations shown on pls. 1A, 1B.]

\begin{tabular}{|c|c|c|c|c|}
\hline \multirow{2}{*}{$\begin{array}{l}\text { Well } \\
\text { number }\end{array}$} & \multicolumn{2}{|c|}{ Water level } & \multirow{2}{*}{$\begin{array}{c}\text { Yield } \\
\text { (gal/min) }\end{array}$} & \multirow{2}{*}{$\begin{array}{c}\text { Remarks } \\
\text { (Depths of geologic units given in feet below land surface) }\end{array}$} \\
\hline & feet & date & & \\
\hline $\begin{array}{l}204 \\
205 \\
206 \\
207 \\
209\end{array}$ & $\begin{array}{r}18 \\
15 \\
25 \\
-- \\
--\end{array}$ & $\begin{array}{c}08-74 \\
05-77 \\
-- \\
-- \\
--\end{array}$ & $\begin{array}{r}-- \\
-- \\
30 \\
4\end{array}$ & $\begin{array}{l}\text { 0-65 sand \& gravel. Hard water. Bailed to bottom at } 30 \mathrm{GPM} \text {. } \\
\text { Hard water, some iron. Negligible DD w/continuous bailing. } \\
\text { Hard water, slight hydrogen sulfide odor. Sand and gravel aquifer? } \\
0-44 \text { dirty gravel w/cobbles. } \\
\text { Abandoned, buried. Household uses spring for supply. Questionable BR depth. }\end{array}$ \\
\hline $\begin{array}{l}210 \\
211 \\
212 \\
213 \\
214\end{array}$ & $\begin{array}{r}75 \\
5 \\
-- \\
12\end{array}$ & $\begin{array}{l}-- \\
-- \\
-- \\
--\end{array}$ & $\begin{array}{r}50 \\
-- \\
15 \\
--\end{array}$ & $\begin{array}{l}\text { Supplies home and } 40 \text { cattle. Hardness reported as } 137 \mathrm{MG} / \mathrm{L} \text {. } \\
\text { Hard water. Owner reports several } \mathrm{ft} \text { of clay near surface. } \\
\text {-- } \\
\text { Owner reports } 0-11 \text { clay; } 11-27 \text { gravel; } 27-30 \text { black sand. } \\
\text { Owner reports soft water. } 2 \mathrm{ft} \text { well point. Well has never failed. }\end{array}$ \\
\hline $\begin{array}{l}215 \\
216 \\
217 \\
218 \\
219\end{array}$ & $\begin{array}{l}37 \\
10 \\
28 \\
50\end{array}$ & $\begin{array}{l}01-81 \\
1955 \\
10-54 \\
1965\end{array}$ & $\begin{array}{l}10 \\
-- \\
35 \\
25\end{array}$ & $\begin{array}{l}0-30 \text { sand; } 30-100 \text { fine sand; } 100-110 \text { gravel. } \\
0-55 \text { sand \& gravel, some silty clay; } 55-104 \text { sand. } \\
0-90 \text { gravel. Water rusty at times. } \\
0-82 \text { dirty gravel; } 82-86 \text { BR. } \\
0-140 \text { gravel. }\end{array}$ \\
\hline $\begin{array}{l}220 \\
221 \\
222 \\
223 \\
224\end{array}$ & $\begin{array}{r}22 \\
12 \\
-- \\
- \\
7\end{array}$ & $\begin{array}{c}1946 \\
-- \\
-- \\
-- \\
1959\end{array}$ & $\begin{array}{r}15 \\
50 \\
35 \\
-- \\
150\end{array}$ & $\begin{array}{l}\text { 0-10 gravel; } 10-100 \mathrm{HP} ; 100-105 \text { fine gravel. } \\
\text { Owner reports } 0-39 \text { sand \& gravel. } \\
\text { 0-70 dirty gravel. } \\
\text { Very hard water. Formerly } 23 \mathrm{ft} \text { deep, failed during the } 1960 \text { drought. } \\
0-55 \text { dirty gravel; } 55-95 \text { sand; } 95 \text { gravelly clay. }\end{array}$ \\
\hline $\begin{array}{l}225 \\
226 \\
227 \\
228 \\
229\end{array}$ & $\begin{array}{c}-- \\
23 \\
--\end{array}$ & $\begin{array}{l}-- \\
09-\overline{-}-63 \\
10-79\end{array}$ & $\begin{array}{r}20 \\
25 \\
8 \\
9\end{array}$ & $\begin{array}{l}\text { Hard water. Well has supplied } 2 \text { homes and barn in the past. } \\
\text { Supplies house and barn. Hardness } 308 \mathrm{MG} / \mathrm{L} \text { reported by owner. } \\
\text { Hard water. } \\
\text { Owner reports } 0-21 \text { sand \& gravel, then some gray clay. } \\
0-20 \text { gravel; } 20-30 \mathrm{HP} ; 30-37 \text { coarse gravel; } 37-55 \text { till?; } 55-68 \text { boulder?; } \\
68-75 \text { HP; } 75 \mathrm{SH} \text {. }\end{array}$ \\
\hline $\begin{array}{l}230 \\
231 \\
232 \\
233 \\
234\end{array}$ & $\begin{array}{l}13 \\
-\overline{35} \\
28 \\
10\end{array}$ & $\begin{array}{l}09-55 \\
10-77 \\
05-28-80 \\
1953\end{array}$ & $\begin{array}{c}20 \\
-- \\
10 \\
-- \\
25\end{array}$ & $\begin{array}{l}0-70 \text { gravel; } 70-75 \text { coarse sand. } \\
\text { Hydrogen sulfide odor and taste. } \\
\text { Adequate supply. Driller reports water enters at } 83 \mathrm{ft} \text {. } \\
\text { Water level measured through vent pipe } 3 \mathrm{ft} \text { above LSD. } \\
0-14 \text { gravel; } 14-40 \text { dirty gravel. Replaced dug well, polluted by detergent. }\end{array}$ \\
\hline $\begin{array}{l}235 \\
236 \\
237 \\
238 \\
239\end{array}$ & $\begin{array}{l}20 \\
-- \\
-- \\
--\end{array}$ & $\begin{array}{c}03-80 \\
- \\
- \\
- \\
--\end{array}$ & $\begin{array}{r}-- \\
3 \\
30 \\
- \\
12\end{array}$ & $\begin{array}{l}\text { Supplies house and farm. Water level measured when pump was removed 3/80. } \\
\text { Hydrogen sulfide odor, taste. Owner uses activated carbon-chlorine. } \\
\text { Driller reports } 0-58 \text { sand \& gravel; } 58-105 \mathrm{SH} \text {. } \\
\text { Well ends in gravel. Pumped at approximately } 60 \mathrm{GPM} \mathrm{w/2} \mathrm{ft} \mathrm{DD.} \\
\text { Owner reports slight hydrogen sulfide odor, taste. }\end{array}$ \\
\hline $\begin{array}{l}240 \\
241 \\
242 \\
243 \\
244\end{array}$ & $\begin{array}{r}6 \\
15 \\
7 \\
25 \\
10\end{array}$ & $\begin{array}{l}05-\overline{-21-80} \\
09-\overline{17-80} \\
--\end{array}$ & $\begin{array}{l}\ddot{8} \\
-- \\
--\end{array}$ & $\begin{array}{l}\text { Hard water, water softener used. } \\
\text { Hard water. Meas. point is vent hole in top of casing, } 1.4 \mathrm{ft} \text { above LSD. } \\
\text { Suction pump. Owner reports nearby } 19 \mathrm{ft} \text { well failed August } 1980 \text {. } \\
0-25 \text { gravel; } 25-29 \text { till; } 29-31 \mathrm{SH} \text {. } \\
\text { Supplies } 3 \text { homes and } 75 \text { cattle. }\end{array}$ \\
\hline $\begin{array}{l}245 \\
246\end{array}$ & $\begin{array}{l}45 \\
15\end{array}$ & $\begin{array}{c}01-74 \\
11-20-80\end{array}$ & 6 & $\begin{array}{l}\text { Drillers bail tests: } 105 \mathrm{ft} \text { water to drill w/, } 186 \mathrm{ft} 5 \mathrm{GPM}, 210 \mathrm{ft} 6 \mathrm{GPM} \text {. } \\
0-56 \text { sand \& gravel; } 56-70 \text { lacust. sed.; } 70-118 \text { silt; } 118-133 \text { sand \& gravel; } \\
\text { 133-137 STS. }\end{array}$ \\
\hline $\begin{array}{l}247 \\
248 \\
249\end{array}$ & 36 & $05-\overline{-} 20-80$ & $\begin{array}{r}8 \\
3 \\
20\end{array}$ & $\begin{array}{l}\text { Driller reports } 0-30 \mathrm{HP} \text { over gravel. Meas. point } 1.1 \mathrm{ft} \text { above LSD. } \\
\text { Owner reports soft water, iron. }\end{array}$ \\
\hline $\begin{array}{l}250 \\
251 \\
252 \\
253 \\
254\end{array}$ & $\begin{array}{r}-- \\
20 \\
88 \\
-- \\
--\end{array}$ & $\begin{array}{l}-- \\
-- \\
05-09-80 \\
-- \\
--\end{array}$ & $\begin{array}{r}25 \\
10 \\
20 \\
17 \\
3\end{array}$ & $\begin{array}{l}-- \\
\text { Owner reports 0-14 gravel; } 14-44 \text { hard clay (till?). Very hard water. } \\
\text { Negligible DD at } 20 \text { GPM. Meas. point is } 1.5 \mathrm{ft} \text { above LSD. } \\
0-6 \text { sand \& gravel; } 6-12 \mathrm{HP} ; 12-32 \text { sand \& gravel. Suction pump. } \\
0-28 \text { sand \& gravel. Owner chlorinates for iron bacteria. Hydrogen sulfide. }\end{array}$ \\
\hline
\end{tabular}


Table 1.--Records of wells and test holes--continued.

[Dashes indicate no data. Locations shown on pls. 1A, 1B.]

\begin{tabular}{|c|c|c|c|c|c|c|c|c|c|c|}
\hline \multirow[b]{2}{*}{$\begin{array}{c}\text { Well } \\
\text { number }\end{array}$} & \multicolumn{3}{|c|}{ Location } & \multirow[b]{2}{*}{ Owner } & \multirow[b]{2}{*}{$\begin{array}{c}\text { Date } \\
\text { drilled }\end{array}$} & \multirow{2}{*}{$\begin{array}{l}\text { Altitude } \\
\text { of land } \\
\text { surface } \\
\text { (feet) }\end{array}$} & \multirow[b]{2}{*}{$\begin{array}{l}\text { Well } \\
\text { depth } \\
\text { (feet) }\end{array}$} & \multirow[b]{2}{*}{$\begin{array}{l}\text { Casing } \\
\text { depth } \\
\text { (feet) }\end{array}$} & \multirow{2}{*}{$\begin{array}{l}\text { Depth } \\
\text { to } \\
\text { bedrock } \\
\text { (feet) }\end{array}$} & \multirow[b]{2}{*}{$\begin{array}{c}\text { Geologic } \\
\text { unit }\end{array}$} \\
\hline & $\begin{array}{c}\text { Lati- } \\
\text { tude } \\
\ldots \ldots\end{array}$ & $\begin{array}{c}\text { Longi- } \\
\text { tude } \\
\ldots, n\end{array}$ & $\begin{array}{l}\text { Accu- } \\
\text { racy }\end{array}$ & & & & & & & \\
\hline $\begin{array}{l}255 \\
256 \\
257 \\
258 \\
259\end{array}$ & $\begin{array}{l}424002 \\
424003 \\
424003 \\
424004 \\
424004\end{array}$ & $\begin{array}{l}0753353 \\
0753044 \\
0753354 \\
0753119 \\
0753357\end{array}$ & $\begin{array}{l}S \\
S \\
S \\
S \\
S\end{array}$ & $\begin{array}{l}\text { Monroe } \\
\text { Bundy } \\
\text { Monroe } \\
\text { Covell } \\
\text { Simons }\end{array}$ & $\begin{array}{l}1975 \\
1945 \\
1969 \\
1969 \\
1975\end{array}$ & $\begin{array}{l}1175 \\
1100 \\
1175 \\
1260 \\
1170\end{array}$ & $\begin{array}{r}29 \\
185 \\
22 \\
168 \\
39\end{array}$ & $\begin{array}{l}29 \\
85 \\
20 \\
28 \\
26\end{array}$ & $\begin{array}{l}-- \\
85 \\
-- \\
28 \\
26\end{array}$ & $\begin{array}{l}\text { KMTC } \\
\text { BDRK } \\
\text { OTSH } \\
\text { BDRK } \\
\text { BDRK }\end{array}$ \\
\hline $\begin{array}{l}260 \\
261 \\
262 \\
263 \\
264\end{array}$ & $\begin{array}{l}424006 \\
424008 \\
424008 \\
424009 \\
424010\end{array}$ & $\begin{array}{l}0753403 \\
0753351 \\
0753355 \\
0753354 \\
0753351\end{array}$ & $\begin{array}{l}S \\
S \\
S \\
S \\
S\end{array}$ & $\begin{array}{l}\text { Nelson } \\
\text { Bennett } \\
\text { McKee } \\
\text { Thompson } \\
\text { Baker }\end{array}$ & $\begin{array}{l}1900 \\
1972 \\
1976 \\
1975 \\
1965\end{array}$ & $\begin{array}{l}1195 \\
1165 \\
1170 \\
1165 \\
1165\end{array}$ & $\begin{array}{l}35 \\
20 \\
48 \\
52 \\
29\end{array}$ & $\begin{array}{l}-- \\
18 \\
31 \\
38 \\
29\end{array}$ & $\begin{array}{c}-- \\
-- \\
31 \\
38 \\
--\end{array}$ & $\begin{array}{l}\text { KMTC } \\
\text { OTSH } \\
\text { BDRK } \\
\text { BDRK } \\
\text { OTSH }\end{array}$ \\
\hline $\begin{array}{l}265 \\
266 \\
267 \\
268 \\
269\end{array}$ & $\begin{array}{l}424012 \\
424014 \\
424014 \\
424020 \\
424021\end{array}$ & $\begin{array}{l}0753405 \\
0753351 \\
0753407 \\
0753351 \\
0753418\end{array}$ & $\begin{array}{l}S \\
S \\
S \\
S \\
S\end{array}$ & $\begin{array}{l}\text { Maynard } \\
\text { Dubois } \\
\text { Tefft } \\
\text { Brokaw } \\
\text { Bames }\end{array}$ & $\begin{array}{l}1960 \\
1978 \\
1963 \\
1950 \\
1964\end{array}$ & $\begin{array}{l}1180 \\
1165 \\
1180 \\
1165 \\
1185\end{array}$ & $\begin{array}{r}25 \\
20 \\
9 \\
15 \\
118\end{array}$ & $\begin{array}{l}-\overline{18} \\
-\overline{13} \\
24\end{array}$ & $\begin{array}{l}-- \\
-- \\
-- \\
--\end{array}$ & $\begin{array}{l}\text { OTSH } \\
\text { OTSH } \\
\text { OTSH } \\
\text { OTSH } \\
\text { BDRK }\end{array}$ \\
\hline $\begin{array}{l}270 \\
271 \\
272 \\
273 \\
274\end{array}$ & $\begin{array}{l}424022 \\
424022 \\
424024 \\
424025 \\
424027\end{array}$ & $\begin{array}{l}0753352 \\
0753418 \\
0751957 \\
0753352 \\
0753343\end{array}$ & $\begin{array}{l}S \\
S \\
F \\
S \\
S\end{array}$ & $\begin{array}{l}\text { Pinckney } \\
\text { Hubbell } \\
\text { Padgett } \\
\text { Blackman } \\
\text { Manwarren }\end{array}$ & $\begin{array}{c}1978 \\
05-01-80 \\
1947 \\
1978 \\
1975\end{array}$ & $\begin{array}{l}1165 \\
1190 \\
1155 \\
1167 \\
1210\end{array}$ & $\begin{array}{r}16 \\
100 \\
63 \\
20 \\
96\end{array}$ & $\begin{array}{l}14 \\
20 \\
63 \\
20 \\
96\end{array}$ & $\begin{array}{l}-- \\
20 \\
-- \\
--\end{array}$ & $\begin{array}{l}\text { OTSH } \\
\text { BDRK } \\
\text { QRNR } \\
\text { OTSH } \\
\text { KMTC }\end{array}$ \\
\hline $\begin{array}{l}275 \\
276 \\
277 \\
278 \\
279\end{array}$ & $\begin{array}{l}424027 \\
424030 \\
424039 \\
424041 \\
424043\end{array}$ & $\begin{array}{l}0753356 \\
0753422 \\
0753428 \\
0752957 \\
0753334\end{array}$ & $\begin{array}{l}S \\
S \\
S \\
F \\
S\end{array}$ & $\begin{array}{l}\text { Shortway } \\
\text { Smyrna Lumber } \\
\text { Brown } \\
\text { Sherburne Village } \\
\text { Hunsicker }\end{array}$ & $\begin{array}{l}1978 \\
1970 \\
1950 \\
1916 \\
1940\end{array}$ & $\begin{array}{l}1172 \\
1180 \\
1170 \\
1050 \\
1305\end{array}$ & $\begin{array}{r}12 \\
20 \\
20 \\
234 \\
180\end{array}$ & $\begin{array}{l}10 \\
18 \\
-- \\
-- \\
--\end{array}$ & $\begin{array}{l}-- \\
-- \\
-- \\
--\end{array}$ & $\begin{array}{l}\text { OTSH } \\
\text { OTSH } \\
\text { OTSH } \\
-- \\
\text { BDRK }\end{array}$ \\
\hline $\begin{array}{l}280 \\
281 \\
282 \\
283 \\
284\end{array}$ & $\begin{array}{l}424044 \\
424049 \\
424058 \\
424102 \\
424102\end{array}$ & $\begin{array}{l}0753002 \\
0753433 \\
0753430 \\
0753008 \\
0753423\end{array}$ & $\begin{array}{l}F \\
S \\
S \\
S \\
S\end{array}$ & $\begin{array}{l}\text { Sherburne Village } \\
\text { Fuller } \\
\text { Schwarting } \\
\text { Taco Inc. } \\
\text { Blanchard }\end{array}$ & $\begin{array}{l}1916 \\
1956 \\
1900 \\
1954 \\
1963\end{array}$ & $\begin{array}{l}1050 \\
1195 \\
1195 \\
1050 \\
1160\end{array}$ & $\begin{array}{r}26 \\
50 \\
17 \\
155 \\
127\end{array}$ & $\begin{array}{l}-- \\
30 \\
- \\
- \\
24\end{array}$ & $\begin{array}{l}-- \\
30 \\
-- \\
--\end{array}$ & $\begin{array}{l}\text { QRNR } \\
\text { BDRK } \\
\text { KMTC } \\
\text { QRNR } \\
\text { BDRK }\end{array}$ \\
\hline $\begin{array}{l}285 \\
286 \\
287 \\
288 \\
289\end{array}$ & $\begin{array}{l}424104 \\
424105 \\
424107 \\
424108 \\
424109\end{array}$ & $\begin{array}{l}0751944 \\
0753401 \\
0752950 \\
0753405 \\
0753359\end{array}$ & $\begin{array}{l}\text { F } \\
\text { S } \\
\text { S } \\
\text { F } \\
\text { S }\end{array}$ & $\begin{array}{l}\text { Root } \\
\text { Baillie Lumber } \\
\text { Sherburne Village } \\
\text { Smyrna Village } \\
\text { Baillie Lumber }\end{array}$ & $\begin{array}{l}1965 \\
1977 \\
1963 \\
1955 \\
1970\end{array}$ & $\begin{array}{l}1150 \\
1140 \\
1055 \\
1155 \\
1137\end{array}$ & $\begin{array}{r}135 \\
75 \\
190 \\
268 \\
28\end{array}$ & $\begin{array}{r}135 \\
52 \\
-- \\
50 \\
26\end{array}$ & $\begin{array}{c}-- \\
52 \\
-- \\
50 \\
--\end{array}$ & $\begin{array}{l}\text { QRNR } \\
\text { BDRK } \\
-- \\
\text { BDRK } \\
\text { OTSH }\end{array}$ \\
\hline $\begin{array}{l}290 \\
291 \\
292 \\
293 \\
294\end{array}$ & $\begin{array}{l}424110 \\
424113 \\
424116 \\
424119 \\
424120\end{array}$ & $\begin{array}{l}0753359 \\
0753016 \\
0753112 \\
0753347 \\
0753346\end{array}$ & $\begin{array}{l}S \\
S \\
S \\
S \\
S\end{array}$ & $\begin{array}{l}\text { Baillie Lumber } \\
\text { Sherburne Village } \\
\text { Losee } \\
\text { Smyrna Village } \\
\text { Smyrna Village }\end{array}$ & $\begin{array}{c}1970 \\
1962 \\
-- \\
04-20-81 \\
04-29-81\end{array}$ & $\begin{array}{l}1137 \\
1055 \\
1075 \\
1128 \\
1127\end{array}$ & $\begin{array}{r}21 \\
85 \\
52 \\
101 \\
56\end{array}$ & $\begin{array}{l}19 \\
-- \\
-- \\
62 \\
47\end{array}$ & $\begin{array}{l}-- \\
-- \\
-- \\
59 \\
--\end{array}$ & $\begin{array}{l}\text { OTSH } \\
\text { QRNR } \\
\text { QRNR } \\
\text { BDRK } \\
\text { KMTC }\end{array}$ \\
\hline $\begin{array}{l}295 \\
296 \\
297 \\
298 \\
299\end{array}$ & $\begin{array}{l}424121 \\
424122 \\
424125 \\
424126 \\
424127\end{array}$ & $\begin{array}{l}0753339 \\
0753104 \\
0753341 \\
0751937 \\
0753332\end{array}$ & $\begin{array}{l}S \\
S \\
S \\
F \\
S\end{array}$ & $\begin{array}{l}\text { Smyrna Village } \\
\text { Boice } \\
\text { Smyrna Village } \\
\text { Gregory } \\
\text { Smyrna Village }\end{array}$ & $\begin{array}{c}04-07-81 \\
1960 \\
04-02-81 \\
1965 \\
04-07-81\end{array}$ & $\begin{array}{l}1123 \\
1060 \\
1130 \\
1190 \\
1119\end{array}$ & $\begin{array}{r}56 \\
158 \\
44 \\
70 \\
32\end{array}$ & $\begin{array}{l}-- \\
-- \\
\overline{70} \\
--\end{array}$ & $\begin{array}{l}-- \\
-- \\
-- \\
--\end{array}$ & $\begin{array}{l}\text { KMTC } \\
\text { QRNR } \\
\text { KMTC } \\
\text { QRNR } \\
\text { LAKE }\end{array}$ \\
\hline $\begin{array}{l}300 \\
301 \\
302 \\
303 \\
304\end{array}$ & $\begin{array}{l}424127 \\
424128 \\
424129 \\
424131 \\
424131\end{array}$ & $\begin{array}{l}0753339 \\
0753336 \\
0753334 \\
0753014 \\
0753205\end{array}$ & $\begin{array}{l}S \\
S \\
S \\
S \\
F\end{array}$ & $\begin{array}{l}\text { Smyrna Village } \\
\text { Smyrna Village } \\
\text { Smyrna Village } \\
\text { Sherburne Village } \\
\text { Sanderson }\end{array}$ & $\begin{array}{c}04-03-81 \\
04-06-81 \\
03-06-81 \\
1963 \\
1948\end{array}$ & $\begin{array}{l}1127 \\
1120 \\
1122 \\
1055 \\
1208\end{array}$ & $\begin{array}{r}48 \\
60 \\
62 \\
123 \\
93\end{array}$ & $\begin{array}{r}-- \\
-- \\
-- \\
111 \\
93\end{array}$ & $\begin{array}{l}48 \\
-- \\
-- \\
-- \\
--\end{array}$ & $\begin{array}{l}\text { BDRK } \\
\text { KMTC } \\
\text { KMTC } \\
\text { QRNR } \\
\text { QRNR }\end{array}$ \\
\hline
\end{tabular}


Table 1.--Records of wells and test holes--continued.

[Dashes indicate no data. Locations shown on pls. 1A, 1B.]

$\begin{array}{cccc}\text { Well } & \text { Water level } & \text { Yield } & \begin{array}{c}\text { Remarks } \\ \text { number }\end{array}\end{array}$

\begin{tabular}{|c|c|c|c|c|}
\hline $\begin{array}{l}255 \\
256 \\
257 \\
258 \\
259\end{array}$ & $\begin{array}{l}-- \\
-- \\
-- \\
-- \\
8\end{array}$ & $\begin{array}{c}-- \\
-- \\
- \\
-- \\
05-07-80\end{array}$ & $\begin{array}{r}-- \\
-- \\
10 \\
8\end{array}$ & $\begin{array}{l}\text { Owner reports } 0-29 \text { sand \& gravel, cobbles. Well can supply } 3-4 \text { homes. } \\
\text { Owner reports } 0-85 \text { sand \& gravel. } \\
\text { Well occasionally fails during droughts. } \\
-- \\
\text { Driller reports } 0-26 \text { sand \& gravel. Meas. point is top of casing, } 0.9 \mathrm{ft} \text { above } \\
\text { LSD. }\end{array}$ \\
\hline $\begin{array}{l}260 \\
261 \\
262 \\
263 \\
264\end{array}$ & $\begin{array}{l}27 \\
15 \\
16 \\
16 \\
10\end{array}$ & $\begin{array}{c}05-06-80 \\
- \\
05-06-80 \\
-- \\
--\end{array}$ & $\begin{array}{c}-. \\
30 \\
12 \\
-- \\
40\end{array}$ & $\begin{array}{l}\text { Hard water, low yield, uses spring. Meas. point is top curb at LSD. } \\
2 \mathrm{ft} \text { drive point. Hard water. Able to pump } 30 \mathrm{GPM} \text { for short periods. } \\
0-25 \text { gravel; } 25-31 \text { till?; } 31-48 \mathrm{SH} \text {. Meas. point is } 1.2 \mathrm{ft} \text { above LSD. } \\
\text { Owner reports well has never failed. Suction pump. } \\
\text { Owner reports yield greater than } 40 \mathrm{GPM} \text { when drilled. }\end{array}$ \\
\hline $\begin{array}{l}265 \\
266 \\
267 \\
268 \\
269\end{array}$ & $\begin{array}{l}9 \\
-- \\
-- \\
8\end{array}$ & $\begin{array}{l}05-06-80 \\
-- \\
05-\overline{-} \\
--\end{array}$ & $\begin{array}{l}-- \\
-- \\
-- \\
-- \\
3\end{array}$ & $\begin{array}{l}\text { Well has never failed. Meas. point is top of stone curbing at LSD. } \\
\text { Suction pump. Owner reports well has never failed. } \\
\text { Hard water. Well is } 2 \text { by } 4 \mathrm{ft} \text { pit in basement floor, fails occasionally. } \\
\text { Meas. point is top of pipe } 1 \mathrm{ft} \text { above basement floor, } 3 \mathrm{ft} \text { below LSD. } \\
\text { Owner reports } 0-24 \text { sand \& gravel. }\end{array}$ \\
\hline $\begin{array}{l}270 \\
271 \\
272 \\
273 \\
274\end{array}$ & $\begin{array}{r}10 \\
11 \\
-- \\
12 \\
71\end{array}$ & $\begin{array}{l}-- \\
05-02-80 \\
-- \\
-- \\
--\end{array}$ & $\begin{array}{r}5 \\
4 \\
20 \\
-- \\
100\end{array}$ & $\begin{array}{l}\text { Owner reports } 18 \text { in. drive point. Hard water. } \\
\text { Driller reports } 0-8 \text { loam; } 8-20 \text { sand \& gravel. Bail test } 4 \mathrm{GPM} \text { w/85 ft DD. } \\
\text { Penetrates mostly gravel. } \\
6 \text { in. drive point. Hydrogen sulfide. Well has never failed. } \\
\text { Owner reports } 0-96 \text { sand \& gravel. Hard water. }\end{array}$ \\
\hline $\begin{array}{l}275 \\
276 \\
277 \\
278 \\
279\end{array}$ & $\begin{array}{l}-- \\
-- \\
-- \\
--\end{array}$ & $\begin{array}{l}-- \\
- \\
-- \\
-- \\
--\end{array}$ & $\begin{array}{l}-- \\
-- \\
-- \\
--\end{array}$ & $\begin{array}{l}\text { Owner reports well occasionally fails during late summer. } \\
\text { Owner reports well has never failed. } \\
\text { Suction pump. Owner reports well fails occasionally during droughts. } \\
\text { Sulfur odor. Formerly flowed } 0.5 \mathrm{ft} \text { above grade. } \\
\text { Supplies home and } 200 \text { cattle. Hydrogen sulfide odor. Shallow rock? }\end{array}$ \\
\hline $\begin{array}{l}280 \\
281 \\
282 \\
283 \\
284\end{array}$ & $\begin{array}{c}-- \\
- \\
16 \\
-- \\
17\end{array}$ & $\begin{array}{l}-- \\
05-\overline{-} \\
05-80 \\
-\overline{-20-80}\end{array}$ & $\begin{array}{r}100 \\
10 \\
60 \\
10\end{array}$ & $\begin{array}{l}\text { 0-2 soil; } 2-17 \text { clay; } 17-26 \text { gravel. } \\
\text { Well depth approximate. } \\
\text { Owner can pump } 10 \text { GPM for } 40 \text { minutes w/negligible DD. } \\
\text {-- } \\
0-24 \text { till; } 24-135 \text { BR. Static WL } 13 \mathrm{ft} \text { less than LSD, Jan. } 1964 .\end{array}$ \\
\hline $\begin{array}{l}285 \\
286 \\
287 \\
288 \\
289\end{array}$ & $\begin{array}{r}40 \\
10 \\
-- \\
-- \\
--\end{array}$ & $\begin{array}{l}1965 \\
05-07-80 \\
-- \\
-- \\
--\end{array}$ & $\begin{array}{r}10 \\
30 \\
-- \\
30 \\
--\end{array}$ & $\begin{array}{l}0-50 \text { fine sand; } 50-70 \text { gravel; } 70-130 \text { fine sand; } 130-135 \text { gravel. } \\
\text { Boiler feed water. Softener used. Meas. point is } 1.1 \mathrm{ft} \text { above LSD. } \\
\text { No water. } \\
\text { Meas. point is } 5 \mathrm{ft} \text { below LSD. Water level about } 11 \mathrm{ft} 5 / 80 \text {. } \\
\text { Supplies washroom. Occasionally fails during late summer. }\end{array}$ \\
\hline $\begin{array}{l}290 \\
291 \\
292 \\
293 \\
294\end{array}$ & $\begin{array}{l}15 \\
-- \\
-- \\
4 \\
5\end{array}$ & $\begin{array}{l}-\overline{1962} \\
-\overline{0} \\
04-20-81 \\
07-02-81\end{array}$ & $\begin{array}{r}-- \\
-- \\
100 \\
150\end{array}$ & 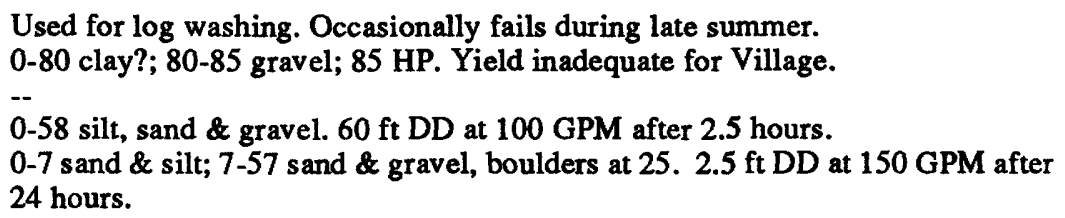 \\
\hline $\begin{array}{l}295 \\
296 \\
297 \\
298 \\
299\end{array}$ & $\begin{array}{c}7 \\
-- \\
10 \\
--\end{array}$ & $\begin{array}{l}04-07-81 \\
-- \\
04-02-81 \\
-- \\
04-07-81\end{array}$ & $\begin{array}{r}200 \\
-- \\
15 \\
--\end{array}$ & $\begin{array}{l}0-7 \text { silt; } 7-15 \text { gravel; } 15-49 \text { fine sand; } 49-56 \text { gravel. } \\
0-5 \text { gravel; } 5-150 \text { blue clay, some gravel; } 150-158 \text { gravel. } \\
0-10 \text { sand; } 10-42 \text { very fine sand \& silt; } 42-44 \text { gravel. } \\
-- \\
0-5 \text { sand; } 5-10 \text { med. to fine gravel; } 10-15 \text { med. to fine sand; } 15-32 \text { fine sand. }\end{array}$ \\
\hline $\begin{array}{l}300 \\
301 \\
302 \\
303 \\
304\end{array}$ & $\begin{array}{r}9 \\
7 \\
10 \\
-- \\
--\end{array}$ & $\begin{array}{l}04-03-81 \\
04-06-81 \\
03-06-81 \\
1964 \\
--\end{array}$ & $\begin{array}{r}-- \\
-- \\
540 \\
8\end{array}$ & $\begin{array}{l}0-17 \text { gravel; } 17-35 \text { very fine to fine sand; } 35-48 \text { med. to fine sand; } 48 \text { BR. } \\
0-10 \text { sand; } 10-20 \text { gravel; } 20-45 \text { fine sand; } 45-59 \text { med. to fine sand; } 59-60 \text { gravel. } \\
0-10 \text { sand \& gravel; } 10-13 \text { gravel; } 13-56 \text { silt; } 56-62 \text { gravel. } \\
0-15 \text { sand \& gravel; } 15-104 \text { blue clay; } 104-123 \text { gravel. Sulfur odor. } \\
0-93 \text { gravel. }\end{array}$ \\
\hline
\end{tabular}


Table 1.--Records of wells and test holes--continued.

[Dashes indicate no data. Locations shown on pls. 1A, 1B.]

\begin{tabular}{|c|c|c|c|c|c|c|c|c|c|c|}
\hline \multirow[b]{2}{*}{$\begin{array}{c}\text { Well } \\
\text { number }\end{array}$} & \multicolumn{3}{|c|}{ Location } & \multirow[b]{2}{*}{ Owner } & \multirow[b]{2}{*}{$\begin{array}{l}\text { Date } \\
\text { drilled }\end{array}$} & \multirow{2}{*}{$\begin{array}{l}\text { Altitude } \\
\text { of land } \\
\text { surface } \\
\text { (feet) }\end{array}$} & \multirow[b]{2}{*}{$\begin{array}{l}\text { Well } \\
\text { depth } \\
\text { (feet) }\end{array}$} & \multirow[b]{2}{*}{$\begin{array}{l}\text { Casing } \\
\text { depth } \\
\text { (feet) }\end{array}$} & \multirow{2}{*}{$\begin{array}{l}\text { Depth } \\
\text { to } \\
\text { bedrock } \\
\text { (feet) }\end{array}$} & \multirow[b]{2}{*}{$\begin{array}{c}\text { Geologic } \\
\text { unit }\end{array}$} \\
\hline & $\begin{array}{l}\text { Lati- } \\
\text { tude } \\
\ldots, n\end{array}$ & $\begin{array}{c}\text { Longi- } \\
\text { tude } \\
\ldots \text { n }\end{array}$ & $\begin{array}{c}\text { Accu- } \\
\text { racy }\end{array}$ & & & & & & & \\
\hline $\begin{array}{l}305 \\
306 \\
307 \\
308 \\
309\end{array}$ & $\begin{array}{l}424135 \\
424137 \\
424138 \\
424138 \\
424139\end{array}$ & $\begin{array}{l}0753319 \\
0753242 \\
0751933 \\
0753208 \\
0753242\end{array}$ & $\begin{array}{l}\text { S } \\
S \\
F \\
F \\
S\end{array}$ & $\begin{array}{l}\text { Wright } \\
\text { Lawrence } \\
\text { Holl } \\
\text { Conley } \\
\text { Blanchard }\end{array}$ & $\begin{array}{c}1950 \\
1955 \\
- \\
1948 \\
1979\end{array}$ & $\begin{array}{l}1103 \\
1127 \\
1185 \\
1182 \\
1110\end{array}$ & $\begin{array}{r}15 \\
120 \\
225 \\
39 \\
98\end{array}$ & $\begin{array}{r}13 \\
60 \\
225 \\
39 \\
56\end{array}$ & $\begin{array}{l}-- \\
60 \\
-- \\
\overline{56}\end{array}$ & $\begin{array}{l}\text { OTSH } \\
\text { BDRK } \\
\text { QRNR } \\
\text { GRVL } \\
\text { BDRK }\end{array}$ \\
\hline $\begin{array}{l}310 \\
311 \\
312 \\
313 \\
314\end{array}$ & $\begin{array}{l}424139 \\
424150 \\
424219 \\
424234 \\
424234\end{array}$ & $\begin{array}{l}0753304 \\
0753324 \\
0753719 \\
0753751 \\
0753753\end{array}$ & $\begin{array}{l}\text { S } \\
\text { S } \\
\mathbf{F} \\
\mathrm{F} \\
\mathrm{F}\end{array}$ & $\begin{array}{l}\text { Lok-N-Logs } \\
\text { Drexler } \\
\text { Rouch } \\
\text { Maltzan } \\
\text { Wynn }\end{array}$ & $\begin{array}{c}1950 \\
11-78 \\
1948 \\
1955 \\
1955\end{array}$ & $\begin{array}{l}1095 \\
1105 \\
1340 \\
1410 \\
1385\end{array}$ & $\begin{array}{l}90 \\
56 \\
63 \\
82 \\
67\end{array}$ & $\begin{array}{l}75 \\
56 \\
63 \\
82 \\
63\end{array}$ & $\begin{array}{l}75 \\
-- \\
60 \\
-- \\
--\end{array}$ & $\begin{array}{l}\text { BDRK } \\
\text { KMTC } \\
\text { BDRK } \\
\text { QRNR } \\
\text { QRNR }\end{array}$ \\
\hline $\begin{array}{l}315 \\
316 \\
317 \\
318 \\
319\end{array}$ & $\begin{array}{l}424248 \\
424251 \\
424309 \\
424310 \\
424310\end{array}$ & $\begin{array}{l}0751859 \\
0751833 \\
0751829 \\
0751833 \\
0754332\end{array}$ & $\begin{array}{l}\text { S } \\
F \\
F \\
F \\
F\end{array}$ & $\begin{array}{l}\text { Holk } \\
\text { Eiholzer } \\
\text { Burdick } \\
\text { Burdick } \\
\text { Nauman }\end{array}$ & $\begin{array}{c}1957 \\
1945 \\
-- \\
1962 \\
--\end{array}$ & $\begin{array}{l}1240 \\
1170 \\
1135 \\
1145 \\
1385\end{array}$ & $\begin{array}{r}137 \\
60 \\
20 \\
34 \\
143\end{array}$ & $\begin{array}{l}86 \\
60 \\
20 \\
34 \\
84\end{array}$ & $\begin{array}{l}86 \\
-- \\
-- \\
-\overline{84}\end{array}$ & $\begin{array}{l}\text { BDRK } \\
\text { OTSH } \\
\text { OTSH } \\
\text { QRNR } \\
\text { BDRK }\end{array}$ \\
\hline $\begin{array}{l}320 \\
321 \\
322 \\
323 \\
324\end{array}$ & $\begin{array}{l}424323 \\
424355 \\
424357 \\
424358 \\
424401\end{array}$ & $\begin{array}{l}0754313 \\
0751806 \\
0751957 \\
0751801 \\
0751756\end{array}$ & $\begin{array}{l}S \\
F \\
S \\
S \\
F\end{array}$ & $\begin{array}{l}\text { Johnson } \\
\text { Maxson } \\
\text { Mikalunas } \\
\text { Maxon } \\
\text { Manchester }\end{array}$ & $\begin{array}{l}-- \\
1940 \\
1965 \\
1965 \\
1961\end{array}$ & $\begin{array}{l}1360 \\
1155 \\
1308 \\
1155 \\
1158\end{array}$ & $\begin{array}{r}47 \\
232 \\
122 \\
200 \\
60\end{array}$ & $\begin{array}{r}47 \\
220 \\
36 \\
170 \\
60\end{array}$ & $\begin{array}{r}-- \\
220 \\
36 \\
170 \\
--\end{array}$ & $\begin{array}{l}\text { QRNR } \\
\text { BDRK } \\
\text { BDRK } \\
\text { BDRK } \\
\text { OTSH }\end{array}$ \\
\hline $\begin{array}{l}325 \\
326 \\
327 \\
328 \\
329\end{array}$ & $\begin{array}{l}424420 \\
421201 \\
421209 \\
421233 \\
421246\end{array}$ & $\begin{array}{l}0753247 \\
0752524 \\
0753120 \\
0752922 \\
0753307\end{array}$ & $\begin{array}{l}\mathrm{S} \\
\mathrm{T} \\
\mathrm{T} \\
\mathrm{T} \\
\mathrm{T}\end{array}$ & $\begin{array}{l}\text { Earlville Village } \\
\text { Hill } \\
\text { Grover } \\
\text { Ketchem } \\
\text { Tanner }\end{array}$ & $\begin{array}{c}1954 \\
05-20-89 \\
07-09-85 \\
08-08-88 \\
09-29-88\end{array}$ & $\begin{array}{l}1085 \\
1760 \\
1110 \\
1460 \\
1050\end{array}$ & $\begin{array}{l}211 \\
255 \\
175 \\
135 \\
140\end{array}$ & $\begin{array}{r}200 \\
20 \\
66 \\
115 \\
30\end{array}$ & $\begin{array}{r}-- \\
20 \\
66 \\
115 \\
30\end{array}$ & $\begin{array}{l}\text { QRNR } \\
\text { BDRK } \\
\text { BDRK } \\
\text { BDRK } \\
\text { BDRK }\end{array}$ \\
\hline $\begin{array}{l}330 \\
331 \\
332 \\
333 \\
334\end{array}$ & $\begin{array}{l}421250 \\
421259 \\
421312 \\
421328 \\
421329\end{array}$ & $\begin{array}{l}0753410 \\
0752521 \\
0752546 \\
0753541 \\
0752601\end{array}$ & $\begin{array}{l}\mathrm{T} \\
\mathrm{T} \\
\mathbf{T} \\
\mathbf{T} \\
\mathrm{T}\end{array}$ & $\begin{array}{l}\text { Kusant } \\
\text { Dematteis } \\
\text { Stearns } \\
\text { Jaindl } \\
\text { Stafford }\end{array}$ & $\begin{array}{l}02-22-89 \\
04-10-89 \\
11-11-86 \\
10-21-85 \\
10-22-85\end{array}$ & $\begin{array}{r}980 \\
1740 \\
1605 \\
1395 \\
1495\end{array}$ & $\begin{array}{l}178 \\
185 \\
250 \\
100 \\
300\end{array}$ & $\begin{array}{l}88 \\
93 \\
94 \\
12 \\
82\end{array}$ & $\begin{array}{l}88 \\
93 \\
94 \\
12 \\
82\end{array}$ & $\begin{array}{l}\text { BDRK } \\
\text { BDRK } \\
\text { BDRK } \\
\text { BDRK } \\
\text { BDRK }\end{array}$ \\
\hline $\begin{array}{l}335 \\
336 \\
337 \\
338 \\
339\end{array}$ & $\begin{array}{l}421356 \\
421521 \\
421522 \\
421524 \\
421543\end{array}$ & $\begin{array}{l}0752905 \\
0752815 \\
0753438 \\
0752722 \\
0754007\end{array}$ & $\begin{array}{l}\mathrm{T} \\
\mathrm{T} \\
\mathrm{T} \\
\mathrm{T} \\
\mathrm{T}\end{array}$ & $\begin{array}{l}\text { Dean } \\
\text { Gabaccia } \\
\text { Trimm } \\
\text { Devries } \\
\text { Gelsomine }\end{array}$ & $\begin{array}{l}05-06-87 \\
10-01-87 \\
05-10-86 \\
02-28-86 \\
01-13-89\end{array}$ & $\begin{array}{l}1130 \\
1020 \\
1340 \\
1115 \\
1625\end{array}$ & $\begin{array}{r}113 \\
227 \\
223 \\
95 \\
160\end{array}$ & $\begin{array}{l}50 \\
52 \\
43 \\
95 \\
45\end{array}$ & $\begin{array}{l}50 \\
52 \\
43 \\
- \\
45\end{array}$ & $\begin{array}{l}\text { BDRK } \\
\text { BDRK } \\
\text { BDRK } \\
\text { QRNR } \\
\text { BDRK }\end{array}$ \\
\hline $\begin{array}{l}340 \\
341 \\
342 \\
343 \\
344\end{array}$ & $\begin{array}{l}421547 \\
421555 \\
421555 \\
421600 \\
421623\end{array}$ & $\begin{array}{l}0752911 \\
0754016 \\
0754902 \\
0754007 \\
0754848\end{array}$ & $\begin{array}{l}\mathrm{T} \\
\mathrm{T} \\
\mathbf{T} \\
\mathrm{T} \\
\mathrm{T}\end{array}$ & $\begin{array}{l}\text { Fernanadez } \\
\text { Johnson } \\
\text { Dedrick } \\
\text { Thomas } \\
\text { Martin }\end{array}$ & $\begin{array}{c}10-27-86 \\
12-01-86 \\
-- \\
04-16-86 \\
--\end{array}$ & $\begin{array}{r}980 \\
1610 \\
960 \\
1585 \\
945\end{array}$ & $\begin{array}{r}55 \\
121 \\
250 \\
200 \\
240\end{array}$ & $\begin{array}{r}55 \\
53 \\
21 \\
21 \\
126\end{array}$ & $\begin{array}{r}-- \\
53 \\
21 \\
21 \\
126\end{array}$ & $\begin{array}{l}\text { QRNR } \\
\text { BDRK } \\
\text { BDRK } \\
\text { BDRK } \\
\text { BDRK }\end{array}$ \\
\hline $\begin{array}{l}345 \\
346 \\
347 \\
349 \\
350\end{array}$ & $\begin{array}{l}421623 \\
421632 \\
421635 \\
421655 \\
421705\end{array}$ & $\begin{array}{l}0754848 \\
0752824 \\
0754626 \\
0754652 \\
0754616\end{array}$ & $\begin{array}{l}\mathbf{T} \\
\mathbf{T} \\
\mathbf{T} \\
\mathbf{T} \\
\mathbf{T}\end{array}$ & $\begin{array}{l}\text { Martin } \\
\text { Young } \\
\text { Markowick } \\
\text { Miller } \\
\text { Wakeman }\end{array}$ & $\begin{array}{c}03-\overline{-28-86} \\
-- \\
-- \\
--\end{array}$ & $\begin{array}{r}945 \\
995 \\
1325 \\
1450 \\
1435\end{array}$ & $\begin{array}{l}200 \\
299 \\
300 \\
226 \\
248\end{array}$ & $\begin{array}{r}67 \\
278 \\
124 \\
20 \\
194\end{array}$ & $\begin{array}{r}67 \\
278 \\
124 \\
20 \\
194\end{array}$ & $\begin{array}{l}\text { BDRK } \\
\text { BDRK } \\
\text { BDRK } \\
\text { BDRK } \\
\text { BDRK }\end{array}$ \\
\hline $\begin{array}{l}351 \\
352 \\
353 \\
354 \\
355\end{array}$ & $\begin{array}{l}421710 \\
421713 \\
421731 \\
421733 \\
421739\end{array}$ & $\begin{array}{l}0754620 \\
0754541 \\
0752855 \\
0752844 \\
0754544\end{array}$ & $\begin{array}{l}\mathrm{T} \\
\mathrm{T} \\
\mathrm{T} \\
\mathrm{T} \\
\mathrm{T}\end{array}$ & $\begin{array}{l}\text { Koerts } \\
\text { Depalma } \\
\text { Catholic Church } \\
\text { Williams } \\
\text { Wade }\end{array}$ & $\begin{array}{c}-- \\
-- \\
-- \\
11-07-88 \\
--\end{array}$ & $\begin{array}{r}1410 \\
1430 \\
995 \\
995 \\
1530\end{array}$ & $\begin{array}{l}225 \\
117 \\
160 \\
150 \\
124\end{array}$ & $\begin{array}{r}94 \\
24 \\
134 \\
110 \\
21\end{array}$ & $\begin{array}{r}94 \\
24 \\
134 \\
110 \\
21\end{array}$ & $\begin{array}{l}\text { BDRK } \\
\text { BDRK } \\
\text { BDRK } \\
\text { BDRK } \\
\text { BDRK }\end{array}$ \\
\hline
\end{tabular}


Table 1.--Records of wells and test holes--continued.

[Dashes indicate no data. Locations shown on pls. 1A, 1B.]

\begin{tabular}{|c|c|c|c|c|}
\hline \multirow{2}{*}{$\begin{array}{c}\text { Well } \\
\text { number }\end{array}$} & \multicolumn{2}{|c|}{ Water level } & \multirow{2}{*}{$\begin{array}{c}\text { Yield } \\
(\mathrm{gal} / \mathrm{min})\end{array}$} & \multirow{2}{*}{$\begin{array}{c}\text { Remarks } \\
\text { (Depths of geologic units given in feet below land surface) }\end{array}$} \\
\hline & feet & date & & \\
\hline $\begin{array}{l}305 \\
306 \\
307 \\
308 \\
309\end{array}$ & $\begin{array}{r}\ddot{40} \\
-- \\
19 \\
14\end{array}$ & $\begin{array}{l}-- \\
-- \\
05-01-48 \\
05-20-80\end{array}$ & $\begin{array}{r}\overline{2} \\
15 \\
15 \\
8\end{array}$ & $\begin{array}{l}\text { Suction pump, drive point. Supplies } 90 \text { cattle. } \\
\text { Owner reports } 0-60 \text { sand \& gravel. Cased off to rock. } \\
\text { 0-39 gravel. } \\
\text { Well to supply future dairy farm. Meas. point is top of casing, } 1.2 \mathrm{ft} \text { above LSD. }\end{array}$ \\
\hline $\begin{array}{l}310 \\
311 \\
312 \\
313 \\
314\end{array}$ & $\begin{array}{r}4 \\
9 \\
33 \\
36 \\
16\end{array}$ & $\begin{array}{l}05-09-80 \\
05-20-80 \\
12-01-48 \\
03-01-55 \\
09-01-55\end{array}$ & $\begin{array}{l}36 \\
18 \\
20 \\
18\end{array}$ & $\begin{array}{l}\text { Meas. point is top of reducer at vent hole, } 2.3 \mathrm{ft} \text { above LSD. } \\
0-56 \text { sand \& gravel. } 19 \mathrm{ft} \text { DD at } 36 \mathrm{GPM} \text {; Meas. point } 2 \mathrm{ft} \text { above LSD. } \\
\text { Rock at } 60 \mathrm{ft} \text {. } \\
\text {-- } \\
\text {-- }\end{array}$ \\
\hline $\begin{array}{l}315 \\
316 \\
317 \\
318 \\
319\end{array}$ & $\begin{array}{l}61 \\
-- \\
-- \\
--\end{array}$ & $\begin{array}{c}08-01-65 \\
-- \\
-- \\
-- \\
--\end{array}$ & $\begin{array}{r}1 \\
-- \\
15 \\
12 \\
2\end{array}$ & $\begin{array}{l}-- \\
-- \\
-- \\
-- \\
--\end{array}$ \\
\hline $\begin{array}{l}320 \\
321 \\
322 \\
323 \\
324\end{array}$ & $\begin{array}{l}-- \\
20 \\
17 \\
40 \\
31\end{array}$ & $\begin{array}{l}01-01-65 \\
08-01-65 \\
05-01-65 \\
11-01-61\end{array}$ & $\begin{array}{r}30 \\
7 \\
9 \\
5 \\
9\end{array}$ & $\begin{array}{l}\text { 0-60 clay; } 60-220 \text { gravel; } 220-232 \text { BR. } \\
\text { 0-36 HP; 36-122 BR. } \\
\text { 0-170 mostly fine, sandy clay; } 170-200 \mathrm{BR} \text {. } \\
\text { 0-10 gravel; } 10-50 \text { clay; } 50-60 \text { gravel. }\end{array}$ \\
\hline $\begin{array}{l}325 \\
326 \\
327 \\
328 \\
329\end{array}$ & $\begin{array}{l}33 \\
-- \\
-- \\
-- \\
--\end{array}$ & $\begin{array}{l}01-01-54 \\
-- \\
-- \\
-- \\
--\end{array}$ & $\begin{array}{r}250 \\
2.5 \\
20 \\
20 \\
5\end{array}$ & $\begin{array}{l}0-35 \text { sand \& gravel, little water; 35-200 clay; 200-211 gravel. Small DD. } \\
-- \\
-- \\
-- \\
--\end{array}$ \\
\hline $\begin{array}{l}330 \\
331 \\
332 \\
333 \\
334\end{array}$ & $\begin{array}{l}-- \\
-- \\
30 \\
--\end{array}$ & $\begin{array}{l}-- \\
-- \\
10-21-85 \\
--\end{array}$ & $\begin{array}{r}15 \\
5 \\
-- \\
10 \\
2\end{array}$ & $\begin{array}{l}-. \\
-- \\
-- \\
-- \\
--\end{array}$ \\
\hline $\begin{array}{l}335 \\
336 \\
337 \\
338 \\
339\end{array}$ & $\begin{array}{l}-- \\
-- \\
-- \\
-- \\
-\end{array}$ & $\begin{array}{l}-- \\
-- \\
-- \\
-- \\
--\end{array}$ & $\begin{array}{r}8 \\
8 \\
10 \\
-- \\
15\end{array}$ & $\begin{array}{l}-- \\
-- \\
-- \\
-- \\
--\end{array}$ \\
\hline $\begin{array}{l}340 \\
341 \\
342 \\
343 \\
344\end{array}$ & $\begin{array}{l}-- \\
-- \\
-- \\
-- \\
--\end{array}$ & $\begin{array}{l}-- \\
-- \\
-- \\
-- \\
--\end{array}$ & $\begin{array}{r}15 \\
-- \\
5 \\
12 \\
1.5\end{array}$ & $\begin{array}{l}-- \\
\text { Artesian well. } \\
-- \\
-- \\
--\end{array}$ \\
\hline $\begin{array}{l}345 \\
346 \\
347 \\
349 \\
350\end{array}$ & $\begin{array}{r}-- \\
20 \\
-- \\
--\end{array}$ & $\begin{array}{c}-- \\
03-28-86 \\
-- \\
-- \\
--\end{array}$ & $\begin{array}{r}5.5 \\
20 \\
4.5 \\
10 \\
4.5\end{array}$ & $\begin{array}{l}-- \\
-- \\
-- \\
-- \\
--\end{array}$ \\
\hline $\begin{array}{l}351 \\
352 \\
353 \\
354 \\
355\end{array}$ & $\begin{array}{l}-- \\
-- \\
-- \\
-- \\
--\end{array}$ & $\begin{array}{l}-- \\
-- \\
- \\
-- \\
--\end{array}$ & $\begin{array}{r}4.5 \\
5 \\
12 \\
-- \\
5.5\end{array}$ & $\begin{array}{l}-- \\
-- \\
-- \\
-- \\
--\end{array}$ \\
\hline
\end{tabular}


Table 1.--Records of wells and test holes--continued.

[Dashes indicate no data. Locations shown on pls. 1A, 1B.]

\begin{tabular}{|c|c|c|c|c|c|c|c|c|c|c|}
\hline \multirow[b]{2}{*}{$\begin{array}{c}\text { Well } \\
\text { number }\end{array}$} & \multicolumn{3}{|c|}{ Location } & \multirow[b]{2}{*}{ Owner } & \multirow[b]{2}{*}{$\begin{array}{c}\text { Date } \\
\text { drilled }\end{array}$} & \multirow{2}{*}{$\begin{array}{l}\text { Altitude } \\
\text { of land } \\
\text { surface } \\
\text { (feet) }\end{array}$} & \multirow[b]{2}{*}{$\begin{array}{l}\text { Well } \\
\text { depth } \\
\text { (feet) }\end{array}$} & \multirow[b]{2}{*}{$\begin{array}{l}\text { Casing } \\
\text { depth } \\
\text { (feet) }\end{array}$} & \multirow{2}{*}{$\begin{array}{l}\text { Depth } \\
\text { to } \\
\text { bedrock } \\
\text { (feet) }\end{array}$} & \multirow[b]{2}{*}{$\begin{array}{c}\text { Geologic } \\
\text { unit }\end{array}$} \\
\hline & $\begin{array}{l}\text { Lati- } \\
\text { tude } \\
\ldots .\end{array}$ & $\begin{array}{c}\text { Longi- } \\
\text { tude } \\
\ldots .\end{array}$ & $\begin{array}{l}\text { Accu- } \\
\text { racy }\end{array}$ & & & & & & & \\
\hline $\begin{array}{l}356 \\
358 \\
360 \\
361 \\
362\end{array}$ & $\begin{array}{l}421740 \\
421742 \\
421746 \\
421746 \\
421747\end{array}$ & $\begin{array}{l}0754620 \\
0754550 \\
0752848 \\
0754549 \\
0754433\end{array}$ & $\begin{array}{l}\mathbf{T} \\
\mathbf{T} \\
\mathbf{T} \\
\mathbf{T} \\
\mathbf{T}\end{array}$ & $\begin{array}{l}\text { Calice } \\
\text { Herrick } \\
\text { Burton } \\
\text { Reich } \\
\text { Montelione }\end{array}$ & $\begin{array}{c}-- \\
\overline{-} \\
10-18-88 \\
-- \\
--\end{array}$ & $\begin{array}{l}1305 \\
1495 \\
1010 \\
1460 \\
1455\end{array}$ & $\begin{array}{r}148 \\
102 \\
98 \\
151 \\
148\end{array}$ & $\begin{array}{l}40 \\
23 \\
98 \\
46 \\
20\end{array}$ & $\begin{array}{l}40 \\
23 \\
-- \\
46 \\
20\end{array}$ & $\begin{array}{l}\text { BDRK } \\
\text { BDRK } \\
\text { QRNR } \\
\text { BDRK } \\
\text { BDRK }\end{array}$ \\
\hline $\begin{array}{l}363 \\
364 \\
365 \\
366 \\
367\end{array}$ & $\begin{array}{l}421753 \\
421801 \\
421803 \\
421803 \\
421806\end{array}$ & $\begin{array}{l}0754110 \\
0754653 \\
0753959 \\
0754008 \\
0752712\end{array}$ & $\begin{array}{l}\mathbf{T} \\
\mathbf{T} \\
\mathbf{T} \\
\mathbf{T} \\
\mathbf{T}\end{array}$ & $\begin{array}{l}\text { Plavak } \\
\text { Gerty } \\
\text { Jennison } \\
\text { Popovitch } \\
\text { Avery }\end{array}$ & $\begin{array}{c}-- \\
-- \\
-- \\
12-30-88\end{array}$ & $\begin{array}{r}1400 \\
930 \\
1550 \\
1545 \\
985\end{array}$ & $\begin{array}{l}223 \\
154 \\
210 \\
184 \\
214\end{array}$ & $\begin{array}{r}109 \\
18 \\
16 \\
14 \\
214\end{array}$ & $\begin{array}{r}109 \\
18 \\
16 \\
14 \\
--\end{array}$ & $\begin{array}{l}\text { BDRK } \\
\text { BDRK } \\
\text { BDRK } \\
\text { BDRK } \\
\text { QRNR }\end{array}$ \\
\hline $\begin{array}{l}368 \\
369 \\
370 \\
371 \\
372\end{array}$ & $\begin{array}{l}421807 \\
421810 \\
421811 \\
421813 \\
421815\end{array}$ & $\begin{array}{l}0752621 \\
0753819 \\
0754651 \\
0754805 \\
0754439\end{array}$ & $\begin{array}{l}\mathbf{T} \\
\mathbf{T} \\
\mathbf{T} \\
\mathbf{T} \\
\mathbf{T}\end{array}$ & $\begin{array}{l}\text { Elder } \\
\text { Sanford } \\
\text { Stiles } \\
\text { Watson } \\
\text { Long }\end{array}$ & $\begin{array}{c}07-13-84 \\
-- \\
-- \\
-- \\
09-11-85\end{array}$ & $\begin{array}{r}1040 \\
1550 \\
915 \\
910 \\
1475\end{array}$ & $\begin{array}{l}239 \\
398 \\
138 \\
184 \\
380\end{array}$ & $\begin{array}{r}139 \\
22 \\
138 \\
184 \\
19\end{array}$ & $\begin{array}{r}139 \\
22 \\
-- \\
-- \\
19\end{array}$ & $\begin{array}{l}\text { BDRK } \\
\text { BDRK } \\
\text { QRNR } \\
\text { QRNR } \\
\text { BDRK }\end{array}$ \\
\hline $\begin{array}{l}373 \\
374 \\
375 \\
376 \\
377\end{array}$ & $\begin{array}{l}421815 \\
421816 \\
421817 \\
421818 \\
421819\end{array}$ & $\begin{array}{l}0754648 \\
0754824 \\
0754855 \\
0752802 \\
0752542\end{array}$ & $\begin{array}{l}\mathbf{T} \\
\mathbf{T} \\
\mathbf{T} \\
\mathbf{T} \\
\mathbf{T}\end{array}$ & $\begin{array}{l}\text { Miller } \\
\text { Crabtree } \\
\text { Wright } \\
\text { Bame } \\
\text { Eskow }\end{array}$ & $\begin{array}{c}-- \\
-- \\
04-10-89 \\
10-03-87\end{array}$ & $\begin{array}{r}920 \\
970 \\
1140 \\
990 \\
995\end{array}$ & $\begin{array}{l}164 \\
198 \\
173 \\
160 \\
211\end{array}$ & $\begin{array}{r}164 \\
44 \\
30 \\
160 \\
211\end{array}$ & $\begin{array}{c}-- \\
44 \\
30 \\
-- \\
--\end{array}$ & $\begin{array}{l}\text { QRNR } \\
\text { BDRK } \\
\text { BDRK } \\
\text { QRNR } \\
\text { QRNR }\end{array}$ \\
\hline $\begin{array}{l}379 \\
380 \\
381 \\
382 \\
383\end{array}$ & $\begin{array}{l}421819 \\
421820 \\
421822 \\
421822 \\
421829\end{array}$ & $\begin{array}{l}0754822 \\
0753042 \\
0752805 \\
0753720 \\
0753109\end{array}$ & $\begin{array}{l}\mathbf{T} \\
\mathbf{T} \\
\mathbf{T} \\
\mathbf{T} \\
\mathbf{T}\end{array}$ & $\begin{array}{l}\text { Shippey } \\
\text { Komp } \\
\text { Bame } \\
\text { Petkash } \\
\text { Haddad }\end{array}$ & $\begin{array}{c}-- \\
06-06-86 \\
12-07-87 \\
05-27-89 \\
05-13-86\end{array}$ & $\begin{array}{r}955 \\
1350 \\
995 \\
1570 \\
1505\end{array}$ & $\begin{array}{l}141 \\
225 \\
151 \\
302 \\
151\end{array}$ & $\begin{array}{r}45 \\
32 \\
115 \\
31 \\
20\end{array}$ & $\begin{array}{r}45 \\
32 \\
115 \\
31 \\
20\end{array}$ & $\begin{array}{l}\text { BDRK } \\
\text { BDRK } \\
\text { BDRK } \\
\text { BDRK } \\
\text { BDRK }\end{array}$ \\
\hline $\begin{array}{l}384 \\
385 \\
386 \\
387 \\
388\end{array}$ & $\begin{array}{l}421829 \\
421832 \\
421833 \\
421834 \\
421835\end{array}$ & $\begin{array}{l}0753731 \\
0754721 \\
0754645 \\
0754717 \\
0754643\end{array}$ & $\begin{array}{l}T \\
T \\
T \\
T \\
T\end{array}$ & $\begin{array}{l}\text { Perry } \\
\text { Slater } \\
\text { Burrowshn } \\
\text { Kane } \\
\text { Burrows }\end{array}$ & $\begin{array}{l}- \\
-- \\
- \\
- \\
--\end{array}$ & $\begin{array}{r}1625 \\
1010 \\
910 \\
1030 \\
915\end{array}$ & $\begin{array}{l}202 \\
192 \\
116 \\
235 \\
120\end{array}$ & $\begin{array}{r}16 \\
192 \\
116 \\
182 \\
120\end{array}$ & $\begin{array}{r}16 \\
-- \\
182 \\
--\end{array}$ & $\begin{array}{l}\text { BDRK } \\
\text { QRNR } \\
\text { QRNR } \\
\text { BDRK } \\
\text { QRNR }\end{array}$ \\
\hline $\begin{array}{l}389 \\
390 \\
391 \\
392 \\
393\end{array}$ & $\begin{array}{l}421839 \\
421840 \\
421848 \\
421852 \\
421852\end{array}$ & $\begin{array}{l}0754544 \\
0752711 \\
0753242 \\
0754751 \\
0754859\end{array}$ & $\begin{array}{l}T \\
T \\
T \\
T \\
T\end{array}$ & $\begin{array}{l}\text { Holcomb } \\
\text { Teelon } \\
\text { Lee } \\
\text { Niggli } \\
\text { Stolarcyk }\end{array}$ & $\begin{array}{c}09-\overline{09-88} \\
05-22-87 \\
-- \\
--\end{array}$ & $\begin{array}{r}1005 \\
1140 \\
1325 \\
910 \\
1100\end{array}$ & $\begin{array}{l}240 \\
132 \\
227 \\
100 \\
248\end{array}$ & $\begin{array}{l}90 \\
42 \\
22 \\
70 \\
22\end{array}$ & $\begin{array}{l}90 \\
42 \\
22 \\
70 \\
22\end{array}$ & $\begin{array}{l}\text { BDRK } \\
\text { BDRK } \\
\text { BDRK } \\
\text { BDRK } \\
\text { BDRK }\end{array}$ \\
\hline $\begin{array}{l}394 \\
395 \\
396 \\
397 \\
398\end{array}$ & $\begin{array}{l}421854 \\
421855 \\
421858 \\
421906 \\
421908\end{array}$ & $\begin{array}{l}0754747 \\
0754752 \\
0754748 \\
0754116 \\
0754252\end{array}$ & $\begin{array}{l}T \\
T \\
T \\
T \\
T\end{array}$ & $\begin{array}{l}\text { Hall } \\
\text { Flynn } \\
\text { Webb } \\
\text { Brant } \\
\text { Pecka }\end{array}$ & $\begin{array}{l}-- \\
-- \\
-- \\
--\end{array}$ & $\begin{array}{r}935 \\
930 \\
940 \\
1410 \\
1180\end{array}$ & $\begin{array}{l}108 \\
110 \\
100 \\
198 \\
300\end{array}$ & $\begin{array}{l}78 \\
85 \\
82 \\
42 \\
20\end{array}$ & $\begin{array}{l}78 \\
85 \\
82 \\
42 \\
20\end{array}$ & $\begin{array}{l}\text { BDRK } \\
\text { BDRK } \\
\text { BDRK } \\
\text { BDRK } \\
\text { BDRK }\end{array}$ \\
\hline $\begin{array}{l}399 \\
400 \\
401 \\
402 \\
403\end{array}$ & $\begin{array}{l}421910 \\
421911 \\
421912 \\
421912 \\
421912\end{array}$ & $\begin{array}{l}0753234 \\
0754254 \\
0754811 \\
0754811 \\
0754952\end{array}$ & $\begin{array}{l}T \\
T \\
T \\
T \\
T\end{array}$ & $\begin{array}{l}\text { Fletcher } \\
\text { Turner } \\
\text { Sergi } \\
\text { Sergi } \\
\text { Gilroy }\end{array}$ & $\begin{array}{c}10-10-85 \\
-- \\
-- \\
-- \\
--\end{array}$ & $\begin{array}{r}1340 \\
1180 \\
995 \\
995 \\
1195\end{array}$ & $\begin{array}{l}247 \\
137 \\
114 \\
148 \\
153\end{array}$ & $\begin{array}{l}31 \\
55 \\
92 \\
96 \\
10\end{array}$ & $\begin{array}{l}31 \\
55 \\
92 \\
96 \\
10\end{array}$ & $\begin{array}{l}\text { BDRK } \\
\text { BDRK } \\
\text { BDRK } \\
\text { BDRK } \\
\text { BDRK }\end{array}$ \\
\hline $\begin{array}{l}404 \\
405 \\
406 \\
407 \\
408\end{array}$ & $\begin{array}{l}421914 \\
421916 \\
421916 \\
421920 \\
421928\end{array}$ & $\begin{array}{l}0754113 \\
0754116 \\
0754647 \\
0753232 \\
0754502\end{array}$ & $\begin{array}{l}T \\
T \\
T \\
T \\
T\end{array}$ & $\begin{array}{l}\text { Palmer } \\
\text { Foran } \\
\text { Neal } \\
\text { Puffer } \\
\text { Gagnon }\end{array}$ & $\begin{array}{l}-- \\
- \\
- \\
- \\
-\end{array}$ & $\begin{array}{r}1310 \\
1285 \\
945 \\
1320 \\
1080\end{array}$ & $\begin{array}{l}155 \\
275 \\
198 \\
205 \\
248\end{array}$ & $\begin{array}{r}11 \\
21 \\
162 \\
20 \\
35\end{array}$ & $\begin{array}{r}11 \\
21 \\
162 \\
20 \\
35\end{array}$ & $\begin{array}{l}\text { BDRK } \\
\text { BDRK } \\
\text { BDRK } \\
\text { BDRK } \\
\text { BDRK }\end{array}$ \\
\hline
\end{tabular}


Table 1.--Records of wells and test holes--continued.

[Dashes indicate no data. Locations shown on pls. 1A, 1B.]

\begin{tabular}{|c|c|c|c|c|}
\hline \multirow{2}{*}{$\begin{array}{c}\text { Well } \\
\text { number }\end{array}$} & \multicolumn{2}{|c|}{ Water level } & \multirow{2}{*}{$\begin{array}{c}\text { Yield } \\
\text { (gal/min) }\end{array}$} & \multirow{2}{*}{$\begin{array}{c}\text { Remarks } \\
\text { (Depths of geologic units given in feet below land surface) }\end{array}$} \\
\hline & feet & date & & \\
\hline $\begin{array}{l}356 \\
358 \\
360 \\
361 \\
362\end{array}$ & $\begin{array}{c}-- \\
-- \\
85 \\
-- \\
--\end{array}$ & $\begin{array}{c}-- \\
-- \\
10-18-88 \\
-- \\
--\end{array}$ & $\begin{array}{r}10 \\
5.5 \\
50 \\
12 \\
6\end{array}$ & $\begin{array}{l}-- \\
-- \\
-- \\
-- \\
--\end{array}$ \\
\hline $\begin{array}{l}363 \\
364 \\
365 \\
366 \\
367\end{array}$ & $\begin{array}{l}-- \\
-- \\
-- \\
--\end{array}$ & $\begin{array}{l}-- \\
-- \\
-- \\
--\end{array}$ & $\begin{array}{r}7 \\
7 \\
5 \\
7.5 \\
15\end{array}$ & $\begin{array}{l}-- \\
-- \\
-- \\
-- \\
--\end{array}$ \\
\hline $\begin{array}{l}368 \\
369 \\
370 \\
371 \\
372\end{array}$ & $\begin{array}{c}50 \\
-- \\
-- \\
-- \\
--\end{array}$ & $\begin{array}{c}07-13-84 \\
-- \\
-- \\
-- \\
--\end{array}$ & $\begin{array}{r}15 \\
2.5 \\
20 \\
20 \\
2.5\end{array}$ & $\begin{array}{l}-- \\
-- \\
-- \\
--\end{array}$ \\
\hline $\begin{array}{l}373 \\
374 \\
375 \\
376 \\
377\end{array}$ & $\begin{array}{l}-- \\
-- \\
-- \\
-- \\
--\end{array}$ & $\begin{array}{l}-- \\
- \\
- \\
- \\
-\end{array}$ & $\begin{array}{r}30 \\
5 \\
10 \\
24 \\
20\end{array}$ & $\begin{array}{l}-- \\
-- \\
-- \\
--\end{array}$ \\
\hline $\begin{array}{l}379 \\
380 \\
381 \\
382 \\
383\end{array}$ & $\begin{array}{c}-- \\
-- \\
-- \\
20\end{array}$ & $\begin{array}{c}- \\
- \\
- \\
05- \\
05-13-86\end{array}$ & $\begin{array}{r}8.5 \\
20 \\
-- \\
2.5 \\
30\end{array}$ & $\begin{array}{l}-- \\
-- \\
-- \\
-- \\
--\end{array}$ \\
\hline $\begin{array}{l}384 \\
385 \\
386 \\
387 \\
388\end{array}$ & $\begin{array}{l}-- \\
-- \\
-- \\
--\end{array}$ & $\begin{array}{l}-- \\
-- \\
-- \\
-- \\
-\end{array}$ & $\begin{array}{r}10 \\
20 \\
20 \\
5 \\
20\end{array}$ & $\begin{array}{l}-- \\
-- \\
-- \\
-- \\
--\end{array}$ \\
\hline $\begin{array}{l}389 \\
390 \\
391 \\
392 \\
393\end{array}$ & $\begin{array}{l}-- \\
-- \\
-- \\
-- \\
--\end{array}$ & $\begin{array}{l}-- \\
- \\
-- \\
-- \\
--\end{array}$ & $\begin{array}{r}2.5 \\
25 \\
10 \\
5 \\
2.5\end{array}$ & $\begin{array}{l}-- \\
-- \\
-- \\
-- \\
--\end{array}$ \\
\hline $\begin{array}{l}394 \\
395 \\
396 \\
397 \\
398\end{array}$ & $\begin{array}{l}-- \\
-- \\
-- \\
-- \\
--\end{array}$ & $\begin{array}{l}-- \\
-- \\
-- \\
--\end{array}$ & $\begin{array}{r}10 \\
15 \\
10 \\
5 \\
2.5\end{array}$ & $\begin{array}{l}-- \\
-- \\
-- \\
--\end{array}$ \\
\hline $\begin{array}{l}399 \\
400 \\
401 \\
402 \\
403\end{array}$ & $\begin{array}{l}-- \\
-- \\
- \\
-- \\
--\end{array}$ & $\begin{array}{l}- \\
- \\
-- \\
-- \\
-\end{array}$ & $\begin{array}{r}4 \\
7.5 \\
5 \\
10 \\
7\end{array}$ & $\begin{array}{l}-- \\
-- \\
-- \\
-- \\
--\end{array}$ \\
\hline $\begin{array}{l}404 \\
405 \\
406 \\
407 \\
408\end{array}$ & $\begin{array}{l}-- \\
-- \\
-- \\
--\end{array}$ & $\begin{array}{l}- \\
-- \\
- \\
- \\
--\end{array}$ & $\begin{array}{r}6 \\
4 \\
8.5 \\
8 \\
5.5\end{array}$ & $\begin{array}{l}-- \\
-- \\
-- \\
-- \\
--\end{array}$ \\
\hline
\end{tabular}


Table 1.--Records of wells and test holes--continued.

[Dashes indicate no data. Locations shown on pls. 1A, 1B.]

\begin{tabular}{|c|c|c|c|c|c|c|c|c|c|c|}
\hline \multirow[b]{2}{*}{$\begin{array}{c}\text { Well } \\
\text { number }\end{array}$} & \multicolumn{3}{|c|}{ Location } & \multirow[b]{2}{*}{ Owner } & \multirow[b]{2}{*}{$\begin{array}{c}\text { Date } \\
\text { drilled }\end{array}$} & \multirow{2}{*}{$\begin{array}{c}\text { Altitude } \\
\text { of land } \\
\text { surface } \\
\text { (feet) }\end{array}$} & \multirow[b]{2}{*}{$\begin{array}{l}\text { Well } \\
\text { depth } \\
\text { (feet) }\end{array}$} & \multirow[b]{2}{*}{$\begin{array}{l}\text { Casing } \\
\text { depth } \\
\text { (feet) }\end{array}$} & \multirow{2}{*}{$\begin{array}{l}\text { Depth } \\
\text { to } \\
\text { bedrock } \\
\text { (feet) }\end{array}$} & \multirow[b]{2}{*}{$\begin{array}{c}\text { Geologic } \\
\text { unit }\end{array}$} \\
\hline & $\begin{array}{c}\text { Lati- } \\
\text { tude } \\
\ldots, n\end{array}$ & $\begin{array}{c}\text { Longi- } \\
\text { tude } \\
\ldots, n\end{array}$ & $\begin{array}{l}\text { Accu- } \\
\text { racy }\end{array}$ & & & & & & & \\
\hline $\begin{array}{l}410 \\
411 \\
412 \\
413 \\
414\end{array}$ & $\begin{array}{l}421933 \\
421933 \\
421936 \\
421936 \\
421939\end{array}$ & $\begin{array}{l}0754450 \\
0754623 \\
0754625 \\
0754659 \\
0754245\end{array}$ & $\begin{array}{l}\mathrm{T} \\
\mathrm{T} \\
\mathrm{T} \\
\mathrm{T} \\
\mathrm{T}\end{array}$ & $\begin{array}{l}\text { Koopman } \\
\text { Parks } \\
\text { Parenteau } \\
\text { Rosekrans } \\
\text { Morales }\end{array}$ & $\begin{array}{l}-- \\
- \\
-- \\
-- \\
--\end{array}$ & $\begin{array}{r}1170 \\
910 \\
912 \\
1045 \\
1200\end{array}$ & $\begin{array}{r}248 \\
80 \\
232 \\
300 \\
230\end{array}$ & $\begin{array}{r}36 \\
72 \\
195 \\
43 \\
25\end{array}$ & $\begin{array}{r}36 \\
72 \\
195 \\
43 \\
25\end{array}$ & $\begin{array}{l}\text { BDRK } \\
\text { BDRK } \\
\text { BDRK } \\
\text { BDRK } \\
\text { BDRK }\end{array}$ \\
\hline $\begin{array}{l}415 \\
416 \\
417 \\
418 \\
419\end{array}$ & $\begin{array}{l}421940 \\
421947 \\
421948 \\
421955 \\
421955\end{array}$ & $\begin{array}{l}0754238 \\
0754959 \\
0754243 \\
0752904 \\
0754446\end{array}$ & $\begin{array}{l}\mathrm{T} \\
\mathrm{T} \\
\mathrm{T} \\
\mathrm{T} \\
\mathrm{T}\end{array}$ & $\begin{array}{l}\text { Ketchum } \\
\text { Badger } \\
\text { Forbes } \\
\text { Tischbein } \\
\text { Walker }\end{array}$ & $\begin{array}{c}-- \\
-- \\
-- \\
10-11-85 \\
--\end{array}$ & $\begin{array}{r}1275 \\
1160 \\
1230 \\
1445 \\
985\end{array}$ & $\begin{array}{l}173 \\
145 \\
198 \\
246 \\
124\end{array}$ & $\begin{array}{r}118 \\
69 \\
49 \\
8 \\
105\end{array}$ & $\begin{array}{r}118 \\
69 \\
49 \\
8 \\
105\end{array}$ & $\begin{array}{l}\text { BDRK } \\
\text { BDRK } \\
\text { BDRK } \\
\text { BDRK } \\
\text { BDRK }\end{array}$ \\
\hline $\begin{array}{l}420 \\
421 \\
423 \\
424 \\
425\end{array}$ & $\begin{array}{l}421958 \\
421958 \\
422000 \\
422003 \\
422004\end{array}$ & $\begin{array}{l}0752932 \\
0754448 \\
0753227 \\
0754648 \\
0754112\end{array}$ & $\begin{array}{l}T \\
T \\
T \\
T \\
T\end{array}$ & $\begin{array}{l}\text { Briordy } \\
\text { Neubauer } \\
\text { Michalec } \\
\text { Smith } \\
\text { Marsh }\end{array}$ & $\begin{array}{c}07-12-89 \\
-- \\
-- \\
1987 \\
--\end{array}$ & $\begin{array}{r}1380 \\
955 \\
1435 \\
1085 \\
1350\end{array}$ & $\begin{array}{r}297 \\
250 \\
300 \\
63 \\
140\end{array}$ & $\begin{array}{l}15 \\
69 \\
82 \\
63 \\
10\end{array}$ & $\begin{array}{r}15 \\
69 \\
82 \\
-- \\
10\end{array}$ & $\begin{array}{l}\text { BDRK } \\
\text { BDRK } \\
\text { BDRK } \\
\text { QRNR } \\
\text { BDRK }\end{array}$ \\
\hline $\begin{array}{l}426 \\
427 \\
428 \\
429 \\
431\end{array}$ & $\begin{array}{l}422004 \\
422006 \\
422008 \\
422010 \\
422017\end{array}$ & $\begin{array}{l}0754752 \\
0754755 \\
0754436 \\
0754818 \\
0752518\end{array}$ & $\begin{array}{l}\mathrm{T} \\
\mathrm{T} \\
\mathrm{T} \\
\mathrm{T} \\
\mathrm{T}\end{array}$ & $\begin{array}{l}\text { Henneman } \\
\text { Dean } \\
\text { Utter } \\
\text { Kingsbury } \\
\text { Smith }\end{array}$ & $\begin{array}{l}-- \\
-- \\
-- \\
--\end{array}$ & $\begin{array}{r}1020 \\
1020 \\
960 \\
1055 \\
1220\end{array}$ & $\begin{array}{l}163 \\
205 \\
199 \\
275 \\
156\end{array}$ & $\begin{array}{r}163 \\
205 \\
89 \\
92 \\
44\end{array}$ & $\begin{array}{c}-- \\
-- \\
89 \\
92 \\
44\end{array}$ & $\begin{array}{l}\text { QRNR } \\
\text { QRNR } \\
\text { BDRK } \\
\text { BDRK } \\
\text { BDRK }\end{array}$ \\
\hline $\begin{array}{l}432 \\
433 \\
434 \\
435 \\
436\end{array}$ & $\begin{array}{l}422020 \\
422026 \\
422032 \\
422038 \\
422039\end{array}$ & $\begin{array}{l}0754713 \\
0754252 \\
0753106 \\
0754254 \\
0754618\end{array}$ & $\begin{array}{l}\mathrm{T} \\
\mathrm{T} \\
\mathrm{T} \\
\mathrm{T} \\
\mathrm{T}\end{array}$ & $\begin{array}{l}\text { Kroon } \\
\text { Eppley } \\
\text { Monroe } \\
\text { Brooks } \\
\text { Cosen }\end{array}$ & $\begin{array}{c}-- \\
-- \\
07-08-86 \\
-- \\
--\end{array}$ & $\begin{array}{r}1190 \\
945 \\
1545 \\
945 \\
1000\end{array}$ & $\begin{array}{r}248 \\
148 \\
300 \\
99 \\
98\end{array}$ & $\begin{array}{l}21 \\
60 \\
10 \\
77 \\
43\end{array}$ & $\begin{array}{l}21 \\
60 \\
10 \\
77 \\
43\end{array}$ & $\begin{array}{l}\text { BDRK } \\
\text { BDRK } \\
\text { BDRK } \\
\text { BDRK } \\
\text { BDRK }\end{array}$ \\
\hline $\begin{array}{l}437 \\
438 \\
439 \\
440 \\
441\end{array}$ & $\begin{array}{l}422041 \\
422047 \\
422055 \\
422059 \\
422104\end{array}$ & $\begin{array}{l}0754257 \\
0754610 \\
0752650 \\
0753339 \\
0753347\end{array}$ & $\begin{array}{l}\mathrm{T} \\
\mathrm{T} \\
\mathrm{T} \\
\mathrm{T} \\
\mathrm{T}\end{array}$ & $\begin{array}{l}\text { Paquette } \\
\text { French } \\
\text { Atten } \\
\text { Cava } \\
\text { Kerezman }\end{array}$ & $\begin{array}{c}-- \\
\overline{--} \\
06-23-88 \\
-\overline{08-88}\end{array}$ & $\begin{array}{r}935 \\
1100 \\
1485 \\
1520 \\
1550\end{array}$ & $\begin{array}{l}148 \\
180 \\
195 \\
145 \\
174\end{array}$ & $\begin{array}{l}55 \\
80 \\
42 \\
58 \\
64\end{array}$ & $\begin{array}{l}55 \\
80 \\
42 \\
58 \\
64\end{array}$ & $\begin{array}{l}\text { BDRK } \\
\text { BDRK } \\
\text { BDRK } \\
\text { BDRK } \\
\text { BDRK }\end{array}$ \\
\hline $\begin{array}{l}442 \\
443 \\
444 \\
445 \\
446\end{array}$ & $\begin{array}{l}422105 \\
422105 \\
422131 \\
422133 \\
422137\end{array}$ & $\begin{array}{l}0754350 \\
0754616 \\
0754614 \\
0752949 \\
0754616\end{array}$ & $\begin{array}{l}T \\
T \\
T \\
T \\
T\end{array}$ & $\begin{array}{l}\text { Sexton } \\
\text { Bates } \\
\text { Burlison } \\
\text { Frank } \\
\text { Adams }\end{array}$ & $\begin{array}{c}-- \\
-- \\
-- \\
04-28-87 \\
--\end{array}$ & $\begin{array}{r}930 \\
1200 \\
1255 \\
1290 \\
1200\end{array}$ & $\begin{array}{r}243 \\
220 \\
268 \\
273 \\
73\end{array}$ & $\begin{array}{l}20 \\
88 \\
60 \\
81 \\
60\end{array}$ & $\begin{array}{l}20 \\
88 \\
60 \\
81 \\
60\end{array}$ & $\begin{array}{l}\text { BDRK } \\
\text { BDRK } \\
\text { BDRK } \\
\text { BDRK } \\
\text { BDRK }\end{array}$ \\
\hline $\begin{array}{l}447 \\
448 \\
449 \\
450 \\
451\end{array}$ & $\begin{array}{l}422143 \\
422144 \\
422148 \\
422148 \\
422148\end{array}$ & $\begin{array}{l}0753519 \\
0754359 \\
0754624 \\
0754624 \\
0754831\end{array}$ & $\begin{array}{l}T \\
T \\
T \\
T \\
T\end{array}$ & $\begin{array}{l}\text { Avila } \\
\text { Lewison } \\
\text { Parks } \\
\text { Parks } \\
\text { Detweiler }\end{array}$ & $\begin{array}{c}09-23-87 \\
-- \\
-- \\
-- \\
--\end{array}$ & $\begin{array}{l}1325 \\
1040 \\
1185 \\
1185 \\
1040\end{array}$ & $\begin{array}{r}90 \\
248 \\
96 \\
100 \\
124\end{array}$ & $\begin{array}{l}21 \\
21 \\
45 \\
50 \\
41\end{array}$ & $\begin{array}{l}21 \\
21 \\
45 \\
50 \\
41\end{array}$ & $\begin{array}{l}\text { BDRK } \\
\text { BDRK } \\
\text { BDRK } \\
\text { BDRK } \\
\text { BDRK }\end{array}$ \\
\hline $\begin{array}{l}452 \\
453 \\
454 \\
455 \\
456\end{array}$ & $\begin{array}{l}422149 \\
422150 \\
422152 \\
422154 \\
422156\end{array}$ & $\begin{array}{l}0754216 \\
0754624 \\
0753015 \\
0754219 \\
0754220\end{array}$ & $\begin{array}{l}T \\
T \\
T \\
T \\
T\end{array}$ & $\begin{array}{l}\text { Roach } \\
\text { Sabin } \\
\text { Laing } \\
\text { Niggli } \\
\text { Sammons }\end{array}$ & $\begin{array}{c}-- \\
07- \\
071-87 \\
-- \\
--\end{array}$ & $\begin{array}{l}1000 \\
1195 \\
1380 \\
1010 \\
1015\end{array}$ & $\begin{array}{l}132 \\
165 \\
352 \\
155 \\
148\end{array}$ & $\begin{array}{r}16 \\
54 \\
110 \\
84 \\
68\end{array}$ & $\begin{array}{r}16 \\
54 \\
110 \\
84 \\
68\end{array}$ & $\begin{array}{l}\text { BDRK } \\
\text { BDRK } \\
\text { BDRK } \\
\text { BDRK } \\
\text { BDRK }\end{array}$ \\
\hline $\begin{array}{l}457 \\
458 \\
460 \\
461 \\
462\end{array}$ & $\begin{array}{l}422157 \\
422203 \\
422214 \\
422223 \\
422228\end{array}$ & $\begin{array}{l}0754651 \\
0754511 \\
0754219 \\
0754622 \\
0754617\end{array}$ & $\begin{array}{l}T \\
T \\
T \\
T \\
T\end{array}$ & $\begin{array}{l}\text { Hanrahan } \\
\text { Rounds } \\
\text { Kresge } \\
\text { Clark } \\
\text { Clark }\end{array}$ & $\begin{array}{l}-- \\
-- \\
-- \\
--\end{array}$ & $\begin{array}{l}1380 \\
1280 \\
1115 \\
1380 \\
1410\end{array}$ & $\begin{array}{l}216 \\
197 \\
169 \\
288 \\
375\end{array}$ & $\begin{array}{r}20 \\
103 \\
92 \\
72 \\
20\end{array}$ & $\begin{array}{r}20 \\
103 \\
92 \\
72 \\
20\end{array}$ & $\begin{array}{l}\text { BDRK } \\
\text { BDRK } \\
\text { BDRK } \\
\text { BDRK } \\
\text { BDRK }\end{array}$ \\
\hline
\end{tabular}


Table 1.--Records of wells and test holes--continued.

[Dashes indicate no data. Locations shown on pls. 1A, 1B.]

\begin{tabular}{|c|c|c|c|c|c|}
\hline \multirow{2}{*}{$\begin{array}{c}\text { Well } \\
\text { number }\end{array}$} & \multicolumn{2}{|c|}{ Water level } & \multirow{2}{*}{$\begin{array}{c}\text { Yield } \\
\text { (gal/min) }\end{array}$} & & \multirow{2}{*}{$\begin{array}{c}\text { Remarks } \\
\text { (Depths of geologic units given in feet below land surface) }\end{array}$} \\
\hline & feet & date & & & \\
\hline 410 & - & -- & 7 & -- & \\
\hline 411 & -- & -- & 9 & -- & \\
\hline 412 & $\ldots$ & $\cdots$ & 8 & $\ldots$ & \\
\hline 413 & $\ldots$ & - & 3 & $\ldots$ & \\
\hline 414 & $\cdots$ & -- & 4 & -- & \\
\hline 415 & -- & -- & 7 & -- & \\
\hline 416 & -- & -- & 8 & -- & \\
\hline 417 & -- & - & 6.5 & - & \\
\hline 418 & $\ldots$ & - & 15 & - & \\
\hline 419 & -- & -- & 15 & -- & \\
\hline 420 & -- & -- & 5 & -- & \\
\hline 421 & - & -- & 5 & -- & \\
\hline 423 & -- & -- & - & - & \\
\hline 424 & -- & -- & 6 & - & \\
\hline 425 & -- & -. & 10 & -- & \\
\hline 426 & - & - & 20 & -- & \\
\hline 427 & - & -- & 10 & - & \\
\hline 428 & $\ldots$ & $\ldots$ & 4 & -- & \\
\hline 429 & - & - & 2.5 & - & \\
\hline 431 & -- & -- & 13 & - & \\
\hline 432 & -. & -- & 5.5 & -. & \\
\hline 433 & -- & -- & 9 & -- & \\
\hline 434 & -- & -. & 2 & - & \\
\hline 435 & $\cdots$ & - & 20 & -. & \\
\hline 436 & -. & -- & 8 & -- & \\
\hline 437 & -- & $\ldots$ & 10 & -- & \\
\hline 438 & - & - & 10 & -- & \\
\hline 439 & -- & -- & - & -- & \\
\hline 440 & -. & -- & 9.5 & -- & \\
\hline 441 & -- & - & 5 & -. & \\
\hline 442 & - & - & 3 & -- & \\
\hline 443 & -. & -- & 12 & -- & \\
\hline 444 & -- & -- & 8 & -- & \\
\hline 445 & .- & -. & 5 & -- & \\
\hline 446 & -- & -- & 10 & -- & \\
\hline 447 & -- & - & 8 & -- & \\
\hline 448 & -. & -- & 3.5 & -- & \\
\hline 449 & -. & -- & 10 & -- & \\
\hline 450 & -- & -- & 10 & -. & \\
\hline 451 & -- & -- & 15 & -- & \\
\hline 452 & - & -- & 8 & - & \\
\hline 453 & -- & - & 12 & -. & \\
\hline 454 & - & -- & 4 & -. & , \\
\hline 455 & -- & -- & 7.5 & -- & \\
\hline 456 & -- & -- & 8 & -- & \\
\hline 457 & -. & -- & 4 & $\ldots$ & \\
\hline 458 & - & -- & 7 & -- & \\
\hline 460 & - & -- & 8 & -- & \\
\hline 461 & -- & - & 2.5 & -- & \\
\hline 462 & -- & -- & 2.5 & -- & \\
\hline
\end{tabular}


Table 1.--Records of wells and test holes--continued.

[Dashes indicate no data. Locations shown on pls. 1A, 1B.]

\begin{tabular}{|c|c|c|c|c|c|c|c|c|c|c|}
\hline \multirow[b]{2}{*}{$\begin{array}{c}\text { Well } \\
\text { number }\end{array}$} & \multicolumn{3}{|c|}{ Location } & \multirow[b]{2}{*}{ Owner } & \multirow[b]{2}{*}{$\begin{array}{l}\text { Date } \\
\text { drilled }\end{array}$} & \multirow{2}{*}{$\begin{array}{c}\text { Altitude } \\
\text { of land } \\
\text { surface } \\
\text { (feet) }\end{array}$} & \multirow[b]{2}{*}{$\begin{array}{l}\text { Well } \\
\text { depth } \\
\text { (feet) }\end{array}$} & \multirow[b]{2}{*}{$\begin{array}{l}\text { Casing } \\
\text { depth } \\
\text { (feet) }\end{array}$} & \multirow{2}{*}{$\begin{array}{c}\text { Depth } \\
\text { to } \\
\text { bedrock } \\
\text { (feet) }\end{array}$} & \multirow[b]{2}{*}{$\begin{array}{l}\text { Geologic } \\
\text { unit }\end{array}$} \\
\hline & $\begin{array}{l}\text { Lati- } \\
\text { tude } \\
\cdots,\end{array}$ & $\begin{array}{c}\text { Longi- } \\
\text { tude } \\
\text {... }\end{array}$ & $\begin{array}{c}\text { Accu- } \\
\text { racy }\end{array}$ & & & & & & & \\
\hline $\begin{array}{l}463 \\
465 \\
466 \\
467 \\
468\end{array}$ & $\begin{array}{l}422232 \\
422234 \\
422237 \\
422240 \\
422242\end{array}$ & $\begin{array}{l}0754611 \\
0754507 \\
0752740 \\
0754953 \\
0752428\end{array}$ & $\begin{array}{l}\mathrm{T} \\
\mathrm{T} \\
\mathrm{T} \\
\mathrm{T} \\
\mathrm{T}\end{array}$ & $\begin{array}{l}\text { Stevens } \\
\text { Basa } \\
\text { Randall } \\
\text { Ives } \\
\text { Cook }\end{array}$ & $\begin{array}{c}-- \\
03-20-86 \\
07-23-85 \\
-- \\
06-05-86\end{array}$ & $\begin{array}{l}1435 \\
1310 \\
1410 \\
1375 \\
1030\end{array}$ & $\begin{array}{l}298 \\
337 \\
200 \\
188 \\
142\end{array}$ & $\begin{array}{r}21 \\
160 \\
55 \\
59 \\
28\end{array}$ & $\begin{array}{r}21 \\
160 \\
55 \\
59 \\
28\end{array}$ & $\begin{array}{l}\text { BDRK } \\
\text { BDRK } \\
\text { BDRK } \\
\text { BDRK } \\
\text { BDRK }\end{array}$ \\
\hline $\begin{array}{l}469 \\
470 \\
471 \\
472 \\
473\end{array}$ & $\begin{array}{l}422242 \\
422242 \\
422243 \\
422245 \\
422252\end{array}$ & $\begin{array}{l}0753457 \\
0754846 \\
0752715 \\
0754510 \\
0755050\end{array}$ & $\begin{array}{l}\mathrm{T} \\
\mathrm{T} \\
\mathrm{T} \\
\mathrm{T} \\
\mathrm{T}\end{array}$ & $\begin{array}{l}\text { Wicklein } \\
\text { Figary } \\
\text { Ives } \\
\text { Foster } \\
\text { Lum }\end{array}$ & $\begin{array}{c}-- \\
02-01-88 \\
-- \\
--\end{array}$ & $\begin{array}{l}1275 \\
1070 \\
1360 \\
1330 \\
1325\end{array}$ & $\begin{array}{l}214 \\
100 \\
197 \\
287 \\
148\end{array}$ & $\begin{array}{r}14 \\
36 \\
50 \\
150 \\
64\end{array}$ & $\begin{array}{r}14 \\
36 \\
50 \\
150 \\
64\end{array}$ & $\begin{array}{l}\text { BDRK } \\
\text { BDRK } \\
\text { BDRK } \\
\text { BDRK } \\
\text { BDRK }\end{array}$ \\
\hline $\begin{array}{l}474 \\
475 \\
476 \\
477 \\
478\end{array}$ & $\begin{array}{l}422255 \\
422256 \\
422256 \\
422258 \\
422303\end{array}$ & $\begin{array}{l}0753442 \\
0752612 \\
0754839 \\
0754130 \\
0755109\end{array}$ & $\begin{array}{l}\mathrm{T} \\
\mathrm{T} \\
\mathrm{T} \\
\mathrm{T} \\
\mathrm{T}\end{array}$ & $\begin{array}{l}\text { Denz } \\
\text { Sutton } \\
\text { Andrus } \\
\text { Lademan } \\
\text { Thurber }\end{array}$ & $\begin{array}{c}10-15-86 \\
08-10-85 \\
-- \\
-- \\
--\end{array}$ & $\begin{array}{l}1180 \\
1330 \\
1040 \\
1250 \\
1335\end{array}$ & $\begin{array}{r}149 \\
200 \\
124 \\
295 \\
45\end{array}$ & $\begin{array}{r}21 \\
150 \\
108 \\
142 \\
45\end{array}$ & $\begin{array}{r}21 \\
150 \\
108 \\
142 \\
--\end{array}$ & $\begin{array}{l}\text { BDRK } \\
\text { BDRK } \\
\text { BDRK } \\
\text { BDRK } \\
\text { QRNR }\end{array}$ \\
\hline $\begin{array}{l}480 \\
481 \\
482 \\
484 \\
485\end{array}$ & $\begin{array}{l}422304 \\
422312 \\
422313 \\
422315 \\
422319\end{array}$ & $\begin{array}{l}0755114 \\
0752518 \\
0755053 \\
0754835 \\
0752622\end{array}$ & $\begin{array}{l}\mathrm{T} \\
\mathrm{T} \\
\mathrm{T} \\
\mathrm{T} \\
\mathrm{T}\end{array}$ & $\begin{array}{l}\text { Sortman } \\
\text { Stewart } \\
\text { Giles } \\
\text { Cook } \\
\text { Sutton }\end{array}$ & $\begin{array}{c}\ddot{0}-87 \\
\ddot{-} \\
\ddot{0} \\
05-10-86\end{array}$ & $\begin{array}{l}1335 \\
1300 \\
1340 \\
1020 \\
1390\end{array}$ & $\begin{array}{r}248 \\
160 \\
81 \\
150 \\
175\end{array}$ & $\begin{array}{r}33 \\
85 \\
81 \\
74 \\
150\end{array}$ & $\begin{array}{r}33 \\
85 \\
-\ddot{74} \\
150\end{array}$ & $\begin{array}{l}\text { BDRK } \\
\text { BDRK } \\
\text { QRNR } \\
\text { BDRK } \\
\text { BDRK }\end{array}$ \\
\hline $\begin{array}{l}486 \\
487 \\
488 \\
489 \\
490\end{array}$ & $\begin{array}{l}422322 \\
422324 \\
422327 \\
422328 \\
422328\end{array}$ & $\begin{array}{l}0752622 \\
0753729 \\
0754918 \\
0752929 \\
0754052\end{array}$ & $\begin{array}{l}\mathrm{T} \\
\mathrm{T} \\
\mathrm{T} \\
\mathrm{T} \\
\mathrm{T}\end{array}$ & $\begin{array}{l}\text { Haller } \\
\text { Hammond } \\
\text { Crow } \\
\text { Ohl } \\
\text { Knapp }\end{array}$ & $\begin{array}{c}11-19-87 \\
04-09-87 \\
\ddot{-} \\
11-12-86 \\
--\end{array}$ & $\begin{array}{l}1390 \\
1060 \\
1170 \\
1610 \\
1340\end{array}$ & $\begin{array}{r}298 \\
44 \\
100 \\
171 \\
173\end{array}$ & $\begin{array}{r}56 \\
44 \\
50 \\
110 \\
21\end{array}$ & $\begin{array}{r}56 \\
-- \\
50 \\
110 \\
21\end{array}$ & $\begin{array}{l}\text { BDRK } \\
\text { QRNR } \\
\text { BDRK } \\
\text { BDRK } \\
\text { BDRK }\end{array}$ \\
\hline $\begin{array}{l}491 \\
492 \\
493 \\
494 \\
495\end{array}$ & $\begin{array}{l}422329 \\
422335 \\
422343 \\
422346 \\
422348\end{array}$ & $\begin{array}{l}0752521 \\
0754125 \\
0754922 \\
0754826 \\
0754116\end{array}$ & $\begin{array}{l}\mathrm{T} \\
\mathrm{T} \\
\mathrm{T} \\
\mathrm{T} \\
\mathrm{T}\end{array}$ & $\begin{array}{l}\text { Carney } \\
\text { Borne } \\
\text { Zimmer } \\
\text { Kenyon } \\
\text { Nichols }\end{array}$ & $\begin{array}{c}08-29-86 \\
-- \\
-- \\
-- \\
--\end{array}$ & $\begin{array}{l}1180 \\
1455 \\
1235 \\
1020 \\
1440\end{array}$ & $\begin{array}{r}136 \\
175 \\
106 \\
75 \\
148\end{array}$ & $\begin{array}{l}75 \\
50 \\
99 \\
68 \\
20\end{array}$ & $\begin{array}{l}75 \\
50 \\
99 \\
68 \\
20\end{array}$ & $\begin{array}{l}\text { BDRK } \\
\text { BDRK } \\
\text { BDRK } \\
\text { BDRK } \\
\text { BDRK }\end{array}$ \\
\hline $\begin{array}{l}496 \\
497 \\
498 \\
499 \\
500\end{array}$ & $\begin{array}{l}422356 \\
422357 \\
422403 \\
422404 \\
422412\end{array}$ & $\begin{array}{l}0753943 \\
0752756 \\
0752400 \\
0754233 \\
0752839\end{array}$ & $\begin{array}{l}\mathrm{T} \\
\mathrm{T} \\
\mathrm{T} \\
\mathrm{T} \\
\mathrm{T}\end{array}$ & $\begin{array}{l}\text { Rubolino } \\
\text { Velasco } \\
\text { Harris } \\
\text { Carr } \\
\text { Simpson }\end{array}$ & $\begin{array}{c}-\overline{0} \\
07-03-87 \\
08-20-88 \\
-- \\
--\end{array}$ & $\begin{array}{l}1225 \\
1680 \\
1150 \\
1300 \\
1050\end{array}$ & $\begin{array}{r}248 \\
350 \\
83 \\
124 \\
56\end{array}$ & $\begin{array}{l}20 \\
23 \\
83 \\
79 \\
56\end{array}$ & $\begin{array}{c}20 \\
23 \\
-- \\
79 \\
--\end{array}$ & $\begin{array}{l}\text { BDRK } \\
\text { BDRK } \\
\text { QRNR } \\
\text { BDRK } \\
\text { QRNR }\end{array}$ \\
\hline $\begin{array}{l}501 \\
502 \\
503 \\
504 \\
505\end{array}$ & $\begin{array}{l}422415 \\
422441 \\
422444 \\
422449 \\
422452\end{array}$ & $\begin{array}{l}0754837 \\
0753012 \\
0753041 \\
0752719 \\
0753013\end{array}$ & $\begin{array}{l}\mathrm{T} \\
\mathrm{T} \\
\mathrm{T} \\
\mathrm{T} \\
\mathrm{T}\end{array}$ & $\begin{array}{l}\text { Connelly } \\
\text { Doyle } \\
\text { Strier } \\
\text { Anderson } \\
\text { Hubbard }\end{array}$ & $\begin{array}{c}-\overline{02-88} \\
103-10-88 \\
11-15-85 \\
10-08-87\end{array}$ & $\begin{array}{l}1055 \\
1560 \\
1635 \\
1600 \\
1560\end{array}$ & $\begin{array}{r}92 \\
104 \\
398 \\
177 \\
121\end{array}$ & $\begin{array}{r}26 \\
40 \\
30 \\
154 \\
10\end{array}$ & $\begin{array}{r}26 \\
40 \\
30 \\
154 \\
10\end{array}$ & $\begin{array}{l}\text { BDRK } \\
\text { BDRK } \\
\text { BDRK } \\
\text { BDRK } \\
\text { BDRK }\end{array}$ \\
\hline $\begin{array}{l}506 \\
507 \\
508 \\
509 \\
510\end{array}$ & $\begin{array}{l}422453 \\
422457 \\
422511 \\
422521 \\
422523\end{array}$ & $\begin{array}{l}0753014 \\
0753148 \\
0753055 \\
0755136 \\
0752709\end{array}$ & $\begin{array}{l}\mathrm{T} \\
\mathrm{T} \\
\mathrm{T} \\
\mathrm{T} \\
\mathrm{T}\end{array}$ & $\begin{array}{l}\text { Hubbard } \\
\text { Swingle } \\
\text { Matlox } \\
\text { Basilius } \\
\text { Bentz }\end{array}$ & $\begin{array}{c}06-01-85 \\
10-10-88 \\
04-08-88 \\
-- \\
05-07-86\end{array}$ & $\begin{array}{l}1565 \\
1530 \\
1745 \\
1270 \\
1700\end{array}$ & $\begin{array}{l}175 \\
150 \\
190 \\
160 \\
197\end{array}$ & $\begin{array}{r}112 \\
10 \\
31 \\
112 \\
21\end{array}$ & $\begin{array}{r}112 \\
10 \\
31 \\
112 \\
21\end{array}$ & $\begin{array}{l}\text { BDRK } \\
\text { BDRK } \\
\text { BDRK } \\
\text { BDRK } \\
\text { BDRK }\end{array}$ \\
\hline $\begin{array}{l}511 \\
512 \\
513 \\
514 \\
515\end{array}$ & $\begin{array}{l}422525 \\
422525 \\
422530 \\
422531 \\
422538\end{array}$ & $\begin{array}{l}0752633 \\
0753802 \\
0752258 \\
0753417 \\
0754244\end{array}$ & $\begin{array}{l}\mathrm{T} \\
\mathrm{T} \\
\mathrm{T} \\
\mathrm{T} \\
\mathrm{T}\end{array}$ & $\begin{array}{l}\text { Sands } \\
\text { Rowe } \\
\text { Zaczeck } \\
\text { Marrone } \\
\text { Nowalk }\end{array}$ & $\begin{array}{c}08-11-86 \\
-- \\
02-01-87 \\
07-02-89 \\
--\end{array}$ & $\begin{array}{r}1585 \\
970 \\
1030 \\
1430 \\
1570\end{array}$ & $\begin{array}{r}100 \\
91 \\
210 \\
235 \\
294\end{array}$ & $\begin{array}{r}22 \\
91 \\
190 \\
100 \\
120\end{array}$ & $\begin{array}{r}22 \\
-\overline{1} \\
190 \\
100 \\
120\end{array}$ & $\begin{array}{l}\text { BDRK } \\
\text { QRNR } \\
\text { BDRK } \\
\text { BDRK } \\
\text { BDRK }\end{array}$ \\
\hline
\end{tabular}


Table 1.--Records of wells and test holes--continued. [Dashes indicate no data. Locations shown on pls. 1A, 1B.]

\begin{tabular}{|c|c|c|c|c|}
\hline \multirow{2}{*}{$\begin{array}{c}\text { Well } \\
\text { number }\end{array}$} & \multicolumn{2}{|c|}{ Water level } & \multirow{2}{*}{$\begin{array}{c}\text { Yield } \\
(\mathrm{gal} / \mathrm{min})\end{array}$} & \multirow{2}{*}{$\begin{array}{c}\text { Remarks } \\
\text { (Depths of geologic units given in feet below land surface) }\end{array}$} \\
\hline & feet & date & & \\
\hline 463 & -. & -- & 2.5 & -- \\
\hline 465 & -- & -- & 10 & -- \\
\hline 466 & -- & -- & 6 & -- \\
\hline 467 & -- & -- & 10 & -- \\
\hline 468 & -- & - & 8 & -- \\
\hline 469 & -- & -- & 9 & -- \\
\hline 470 & -- & -- & 5 & -- \\
\hline 471 & -- & -- & 6 & -- \\
\hline 472 & -- & -- & 6 & -- \\
\hline 473 & -- & -- & 10 & -- \\
\hline 474 & -- & -- & -- & -- \\
\hline 475 & -- & -- & -- & -- \\
\hline 476 & -- & -- & 15 & -- \\
\hline 477 & -- & -- & 15 & -. \\
\hline 478 & -- & -- & 10 & -- \\
\hline 480 & -- & -. & 4.5 & -- \\
\hline 481 & - & -- & 10 & -- \\
\hline 482 & -- & -. & 30 & -- \\
\hline 484 & -- & -- & 5 & -- \\
\hline 485 & -- & -- & 15 & -. \\
\hline 486 & - & -- & 3.5 & -- \\
\hline 487 & 25 & $04-09-87$ & 30 & -- \\
\hline 488 & - & -- & 6 & -. \\
\hline 489 & -- & -- & -- & -- \\
\hline 490 & -- & -- & 8 & -- \\
\hline 491 & -- & -- & 10 & - \\
\hline 492 & -- & -- & 6.5 & -. \\
\hline 493 & -- & -- & 20 & -- \\
\hline 494 & -- & -- & 20 & -- \\
\hline 495 & -- & -- & 5 & -- \\
\hline 496 & - & -- & 6.5 & -- \\
\hline 497 & -- & -- & 2 & -- \\
\hline 498 & -- & -. & 6 & -- \\
\hline 499 & -- & -- & 10 & -- \\
\hline 500 & -- & -- & 10 & -- \\
\hline 501 & -- & -. & 5.5 & -- \\
\hline 502 & -- & -- & 15 & -- \\
\hline 503 & -- & -- & 3 & -- \\
\hline 504 & 3 & $11-15-85$ & 30 & -- \\
\hline 505 & -- & - & 12 & -- \\
\hline 506 & -- & -- & 15 & -- \\
\hline 507 & -- & -- & 8 & -- \\
\hline 508 & -- & -- & 20 & -- \\
\hline 509 & -- & -- & 4 & -- \\
\hline 510 & -- & -- & 4 & -- \\
\hline 511 & -- & -- & -- & -- \\
\hline 512 & -- & -- & 12 & -. \\
\hline 513 & -. & -. & 20 & -- \\
\hline 514 & -- & -- & 9 & -- \\
\hline 515 & -- & -- & 6 & -- \\
\hline
\end{tabular}


Table 1.--Records of wells and test holes--continued.

[Dashes indicate no data. Locations shown on pls. 1A, 1B.]

\begin{tabular}{|c|c|c|c|c|c|c|c|c|c|c|}
\hline \multirow[b]{2}{*}{$\begin{array}{c}\text { Well } \\
\text { number }\end{array}$} & \multicolumn{3}{|c|}{ Location } & \multirow[b]{2}{*}{ Owner } & \multirow{2}{*}{\multicolumn{2}{|c|}{$\begin{array}{c}\text { Altitude } \\
\text { of land } \\
\text { surface } \\
\text { (feet) }\end{array}$}} & \multirow[b]{2}{*}{$\begin{array}{l}\text { Well } \\
\text { depth } \\
\text { (feet) }\end{array}$} & \multirow{2}{*}{\multicolumn{2}{|c|}{$\begin{array}{c}\text { Depth } \\
\text { to } \\
\text { bedrock } \\
\text { (feet) }\end{array}$}} & \multirow[b]{2}{*}{$\begin{array}{c}\text { Geologic } \\
\text { unit }\end{array}$} \\
\hline & $\begin{array}{l}\text { Lati- } \\
\text { tude } \\
\ldots \ldots\end{array}$ & $\begin{array}{c}\text { Longi- } \\
\text { tude } \\
\ldots \text {.." }\end{array}$ & $\begin{array}{l}\text { Accu- } \\
\text { racy }\end{array}$ & & & & & & & \\
\hline $\begin{array}{l}516 \\
517 \\
518 \\
519 \\
520\end{array}$ & $\begin{array}{l}422539 \\
422545 \\
422548 \\
422548 \\
422549\end{array}$ & $\begin{array}{l}0752838 \\
0752708 \\
0752824 \\
0754108 \\
0753834\end{array}$ & $\begin{array}{l}\mathrm{T} \\
\mathbf{T} \\
\mathbf{T} \\
\mathrm{T} \\
\mathrm{T}\end{array}$ & $\begin{array}{l}\text { Green } \\
\text { Stock } \\
\text { Beckwith } \\
\text { Fitch } \\
\text { Gardner }\end{array}$ & $\begin{array}{c}11-05-88 \\
08-23-85 \\
03-11-88 \\
09-15-86 \\
--\end{array}$ & $\begin{array}{l}1645 \\
1665 \\
1305 \\
1540 \\
1335\end{array}$ & $\begin{array}{l}198 \\
460 \\
248 \\
198 \\
102\end{array}$ & $\begin{array}{l}23 \\
12 \\
50 \\
27 \\
38\end{array}$ & $\begin{array}{l}23 \\
12 \\
50 \\
27 \\
38\end{array}$ & $\begin{array}{l}\text { BDRK } \\
\text { BDRK } \\
\text { BDRK } \\
\text { BDRK } \\
\text { BDRK }\end{array}$ \\
\hline $\begin{array}{l}523 \\
524 \\
525 \\
526 \\
527\end{array}$ & $\begin{array}{l}422613 \\
422620 \\
422620 \\
422622 \\
422624\end{array}$ & $\begin{array}{l}0753505 \\
0753634 \\
0754915 \\
0753547 \\
0753349\end{array}$ & $\begin{array}{l}\mathbf{T} \\
\mathbf{T} \\
\mathbf{T} \\
\mathbf{T} \\
\mathbf{T}\end{array}$ & $\begin{array}{l}\text { Perry } \\
\text { Burrell } \\
\text { Littlefield } \\
\text { Oralls } \\
\text { Courtwright }\end{array}$ & $\begin{array}{c}-\overline{-} \\
02-11-88 \\
-- \\
-- \\
--\end{array}$ & $\begin{array}{r}1200 \\
1070 \\
1430 \\
985 \\
1570\end{array}$ & $\begin{array}{l}132 \\
250 \\
173 \\
198 \\
115\end{array}$ & $\begin{array}{l}20 \\
55 \\
49 \\
41 \\
25\end{array}$ & $\begin{array}{l}20 \\
55 \\
49 \\
41 \\
25\end{array}$ & $\begin{array}{l}\text { BDRK } \\
\text { BDRK } \\
\text { BDRK } \\
\text { BDRK } \\
\text { BDRK }\end{array}$ \\
\hline $\begin{array}{l}528 \\
529 \\
530 \\
531 \\
532\end{array}$ & $\begin{array}{l}422625 \\
422626 \\
422627 \\
422628 \\
422628\end{array}$ & $\begin{array}{l}0753213 \\
0753344 \\
0753226 \\
0753234 \\
0754631\end{array}$ & $\begin{array}{l}\mathbf{T} \\
\mathbf{T} \\
\mathbf{T} \\
\mathbf{T} \\
\mathbf{T}\end{array}$ & $\begin{array}{l}\text { Gukelberger } \\
\text { Sherman } \\
\text { Chiusano } \\
\text { Granata } \\
\text { Gross }\end{array}$ & $\begin{array}{c}-- \\
03-11-87 \\
-- \\
-- \\
--\end{array}$ & $\begin{array}{l}1550 \\
1560 \\
1535 \\
1570 \\
1120\end{array}$ & $\begin{array}{l}172 \\
118 \\
223 \\
220 \\
125\end{array}$ & $\begin{array}{l}95 \\
35 \\
97 \\
74 \\
20\end{array}$ & $\begin{array}{l}95 \\
35 \\
97 \\
74 \\
20\end{array}$ & $\begin{array}{l}\text { BDRK } \\
\text { BDRK } \\
\text { BDRK } \\
\text { BDRK } \\
\text { BDRK }\end{array}$ \\
\hline $\begin{array}{l}533 \\
534 \\
535 \\
536 \\
537\end{array}$ & $\begin{array}{l}422638 \\
422639 \\
422642 \\
422646 \\
422648\end{array}$ & $\begin{array}{l}0753636 \\
0753309 \\
0753247 \\
0753443 \\
0753247\end{array}$ & $\begin{array}{l}\mathbf{T} \\
\mathbf{T} \\
\mathbf{T} \\
\mathbf{T} \\
\mathbf{T}\end{array}$ & $\begin{array}{l}\text { Podraza } \\
\text { Robinson } \\
\text { Lawrence } \\
\text { Root } \\
\text { Yearwood }\end{array}$ & $\begin{array}{c}-\overline{0} \\
07-08-89 \\
10-20-85 \\
-- \\
05-11-88\end{array}$ & $\begin{array}{l}1185 \\
1595 \\
1590 \\
1065 \\
1595\end{array}$ & $\begin{array}{l}135 \\
282 \\
223 \\
213 \\
138\end{array}$ & $\begin{array}{r}51 \\
22 \\
17 \\
187 \\
16\end{array}$ & $\begin{array}{r}51 \\
22 \\
17 \\
187 \\
16\end{array}$ & $\begin{array}{l}\text { BDRK } \\
\text { BDRK } \\
\text { BDRK } \\
\text { BDRK } \\
\text { BDRK }\end{array}$ \\
\hline $\begin{array}{l}538 \\
539 \\
540 \\
541 \\
542\end{array}$ & $\begin{array}{l}422650 \\
422654 \\
422656 \\
422658 \\
422659\end{array}$ & $\begin{array}{l}0753243 \\
0753300 \\
0753714 \\
0753611 \\
0754738\end{array}$ & $\begin{array}{l}\mathbf{T} \\
\mathbf{T} \\
\mathbf{T} \\
\mathbf{T} \\
\mathbf{T}\end{array}$ & $\begin{array}{l}\text { Jamieson } \\
\text { Endres } \\
\text { Wall } \\
\text { Adron } \\
\text { Zendler }\end{array}$ & $\begin{array}{c}-- \\
07-\overline{-04-85} \\
-- \\
--\end{array}$ & $\begin{array}{l}1620 \\
1625 \\
1265 \\
1200 \\
1380\end{array}$ & $\begin{array}{l}114 \\
266 \\
197 \\
117 \\
224\end{array}$ & $\begin{array}{r}7 \\
10 \\
85 \\
74 \\
32\end{array}$ & $\begin{array}{r}7 \\
10 \\
85 \\
74 \\
32\end{array}$ & $\begin{array}{l}\text { BDRK } \\
\text { BDRK } \\
\text { BDRK } \\
\text { BDRK } \\
\text { BDRK }\end{array}$ \\
\hline $\begin{array}{l}543 \\
544 \\
545 \\
546 \\
547\end{array}$ & $\begin{array}{l}422705 \\
422709 \\
422719 \\
422719 \\
422720\end{array}$ & $\begin{array}{l}0753404 \\
0753054 \\
0752627 \\
0753850 \\
0755017\end{array}$ & $\begin{array}{l}\mathrm{T} \\
\mathrm{T} \\
\mathrm{T} \\
\mathrm{T} \\
\mathrm{T}\end{array}$ & $\begin{array}{l}\text { Hestor } \\
\text { Kinter } \\
\text { Mertz } \\
\text { Hubschmitt } \\
\text { Warren }\end{array}$ & $\begin{array}{c}-- \\
11-08-88 \\
10-14-88 \\
12-09-88 \\
--\end{array}$ & $\begin{array}{l}1090 \\
1420 \\
1645 \\
1585 \\
1450\end{array}$ & $\begin{array}{l}105 \\
338 \\
165 \\
195 \\
150\end{array}$ & $\begin{array}{l}26 \\
60 \\
21 \\
30 \\
67\end{array}$ & $\begin{array}{l}26 \\
60 \\
21 \\
30 \\
67\end{array}$ & $\begin{array}{l}\text { BDRK } \\
\text { BDRK } \\
\text { BDRK } \\
\text { BDRK } \\
\text { BDRK }\end{array}$ \\
\hline $\begin{array}{l}548 \\
549 \\
550 \\
552 \\
553\end{array}$ & $\begin{array}{l}422723 \\
422728 \\
422731 \\
422741 \\
422742\end{array}$ & $\begin{array}{l}0752345 \\
0753854 \\
0753855 \\
0753255 \\
0754209\end{array}$ & $\begin{array}{l}\mathbf{T} \\
\mathbf{T} \\
\mathbf{T} \\
\mathbf{T} \\
\mathbf{T}\end{array}$ & $\begin{array}{l}\text { Lund } \\
\text { Hansen } \\
\text { Oxford Rod \& Gun } \\
\text { Beck } \\
\text { Leach }\end{array}$ & $\begin{array}{c}08-20-85 \\
-- \\
03-25-86 \\
05-13-87 \\
--\end{array}$ & $\begin{array}{r}1040 \\
1580 \\
1580 \\
995 \\
1470\end{array}$ & $\begin{array}{l}180 \\
275 \\
297 \\
178 \\
146\end{array}$ & $\begin{array}{r}180 \\
25 \\
8 \\
178 \\
96\end{array}$ & $\begin{array}{r}25 \\
8 \\
-- \\
96\end{array}$ & $\begin{array}{l}\text { QRNR } \\
\text { BDRK } \\
\text { BDRK } \\
\text { QRNR } \\
\text { BDRK }\end{array}$ \\
\hline $\begin{array}{l}554 \\
555 \\
556 \\
557 \\
558\end{array}$ & $\begin{array}{l}422745 \\
422745 \\
422749 \\
422751 \\
422753\end{array}$ & $\begin{array}{l}0753121 \\
0753127 \\
0754545 \\
0753004 \\
0753901\end{array}$ & $\begin{array}{l}\mathbf{T} \\
\mathbf{T} \\
\mathbf{T} \\
\mathbf{T} \\
\mathbf{T}\end{array}$ & $\begin{array}{l}\text { Yaiser } \\
\text { Blom } \\
\text { Roach } \\
\text { Drexler } \\
\text { Williams }\end{array}$ & $\begin{array}{c}-- \\
-- \\
09-19-86 \\
--\end{array}$ & $\begin{array}{l}1130 \\
1135 \\
1320 \\
1330 \\
1575\end{array}$ & $\begin{array}{l}194 \\
124 \\
198 \\
148 \\
104\end{array}$ & $\begin{array}{r}72 \\
61 \\
123 \\
31 \\
22\end{array}$ & $\begin{array}{r}72 \\
61 \\
123 \\
31 \\
22\end{array}$ & $\begin{array}{l}\text { BDRK } \\
\text { BDRK } \\
\text { BDRK } \\
\text { BDRK } \\
\text { BDRK }\end{array}$ \\
\hline $\begin{array}{l}559 \\
560 \\
561 \\
562 \\
563\end{array}$ & $\begin{array}{l}422757 \\
422759 \\
422803 \\
422804 \\
422806\end{array}$ & $\begin{array}{l}0754606 \\
0755054 \\
0753913 \\
0752950 \\
0754106\end{array}$ & $\begin{array}{l}\mathbf{T} \\
\mathbf{T} \\
\mathbf{T} \\
\mathbf{T} \\
\mathbf{T}\end{array}$ & $\begin{array}{l}\text { Eirman } \\
\text { Maroney } \\
\text { Blackman } \\
\text { Moorehead } \\
\text { Frank }\end{array}$ & $\begin{array}{c}12-16-85 \\
-- \\
11-23-87 \\
06-18-86 \\
08-11-88\end{array}$ & $\begin{array}{l}1445 \\
1495 \\
1560 \\
1430 \\
1545\end{array}$ & $\begin{array}{l}251 \\
175 \\
200 \\
248 \\
197\end{array}$ & $\begin{array}{r}170 \\
93 \\
18 \\
21 \\
40\end{array}$ & $\begin{array}{r}170 \\
93 \\
18 \\
21 \\
40\end{array}$ & $\begin{array}{l}\text { BDRK } \\
\text { BDRK } \\
\text { BDRK } \\
\text { BDRK } \\
\text { BDRK }\end{array}$ \\
\hline $\begin{array}{l}564 \\
565 \\
566 \\
569 \\
570\end{array}$ & $\begin{array}{l}422808 \\
422810 \\
422810 \\
422831 \\
422832\end{array}$ & $\begin{array}{l}0753832 \\
0753638 \\
0754525 \\
0753227 \\
0753244\end{array}$ & $\begin{array}{l}\mathbf{T} \\
\mathbf{T} \\
\mathbf{T} \\
\mathbf{T} \\
\mathbf{T}\end{array}$ & $\begin{array}{l}\text { Bartle } \\
\text { Bahn } \\
\text { Brown } \\
\text { Smith } \\
\text { Quinn }\end{array}$ & $\begin{array}{c}09-15-86 \\
09-28-85 \\
-- \\
-- \\
--\end{array}$ & $\begin{array}{l}1315 \\
1580 \\
1200 \\
1025 \\
1040\end{array}$ & $\begin{array}{l}223 \\
340 \\
148 \\
317 \\
148\end{array}$ & $\begin{array}{r}63 \\
16 \\
32 \\
312 \\
42\end{array}$ & $\begin{array}{r}63 \\
16 \\
32 \\
312 \\
42\end{array}$ & $\begin{array}{l}\text { BDRK } \\
\text { BDRK } \\
\text { BDRK } \\
\text { BDRK } \\
\text { BDRK }\end{array}$ \\
\hline
\end{tabular}


Table 1.--Records of wells and test holes--continued.

[Dashes indicate no data. Locations shown on pls. 1A, 1B.]

\begin{tabular}{|c|c|c|c|c|}
\hline \multirow{2}{*}{$\begin{array}{c}\text { Well } \\
\text { number }\end{array}$} & \multicolumn{2}{|c|}{ Water level } & \multirow{2}{*}{$\underset{\text { (gal/min) }}{\text { Yield }}$} & \multirow{2}{*}{$\begin{array}{c}\text { Remarks } \\
\text { (Depths of geologic units given in feet below land surface) }\end{array}$} \\
\hline & feet & date & & \\
\hline 516 & -- & - & 6 & -- \\
\hline 517 & -- & -- & -- & - \\
\hline 518 & -- & -- & 5 & -- \\
\hline 519 & -- & -- & 10 & -- \\
\hline 520 & -- & -- & 6 & -- \\
\hline 523 & -- & -- & 10 & -- \\
\hline 524 & -- & -- & 4 & -- \\
\hline 525 & -- & .- & 5 & -- \\
\hline 526 & -- & -- & 5 & -- \\
\hline 527 & -- & -- & 5 & -- \\
\hline 528 & -- & -- & 5 & -. \\
\hline 529 & -- & -. & 30 & -- \\
\hline 530 & -- & -- & 10 & -. \\
\hline 531 & -- & - & 10 & -- \\
\hline 532 & -- & -- & 20 & -- \\
\hline 533 & -- & -. & 12 & -. \\
\hline 534 & -- & -. & 6 & -. \\
\hline 535 & -- & -- & 4 & -- \\
\hline 536 & -- & -- & 8 & -- \\
\hline 537 & -- & -- & 15 & -- \\
\hline 538 & -. & -- & 8.5 & -- \\
\hline 539 & -- & -- & 5.5 & -. \\
\hline 540 & -- & -- & 20 & -- \\
\hline 541 & -- & -- & 15 & -- \\
\hline 542 & -- & -- & 6 & -- \\
\hline 543 & -- & -- & 11 & -- \\
\hline 544 & -- & -- & 2.5 & .- \\
\hline 545 & -- & -- & 8 & -- \\
\hline 546 & -- & -- & 10 & -- \\
\hline 547 & -- & -- & 20 & -- \\
\hline 548 & 20 & $08-20-85$ & 20 & .- \\
\hline 549 & -- & -- & 4.5 & -- \\
\hline 550 & -- & -- & 4 & -- \\
\hline 552 & -- & -- & 20 & -- \\
\hline 553 & -- & - & 10 & -- \\
\hline 554 & -- & -- & 4.5 & -- \\
\hline 555 & -- & -- & 30 & -- \\
\hline 556 & -- & -- & 17.5 & -- \\
\hline 557 & -- & -- & -- & -- \\
\hline 558 & -- & -- & 9.5 & -- \\
\hline 559 & -- & -- & 7 & -- \\
\hline 560 & -- & -- & 20 & -- \\
\hline 561 & -- & -- & 2 & -. \\
\hline 562 & -- & -- & 4 & -- \\
\hline 563 & -- & -- & 36 & -- \\
\hline 564 & -- & -- & 5 & -- \\
\hline 565 & - & -- & 2 & - \\
\hline 566 & -- & -- & 10 & -- \\
\hline 569 & -- & -- & 15 & -- \\
\hline 570 & -- & -- & 6 & -. \\
\hline
\end{tabular}


Table 1.--Records of wells and test holes--continued.

[Dashes indicate no data. Locations shown on pls. 1A, 1B.]

\begin{tabular}{|c|c|c|c|c|c|c|c|c|c|c|}
\hline \multirow[b]{2}{*}{$\begin{array}{l}\text { Well } \\
\text { number }\end{array}$} & \multicolumn{3}{|c|}{ Location } & \multirow[b]{2}{*}{ Owner } & \multirow[b]{2}{*}{$\begin{array}{c}\text { Date } \\
\text { drilled }\end{array}$} & \multirow{2}{*}{$\begin{array}{c}\text { Altitude } \\
\text { of land } \\
\text { surface } \\
\text { (feet) }\end{array}$} & \multirow[b]{2}{*}{$\begin{array}{l}\text { Well } \\
\text { depth } \\
\text { (feet) }\end{array}$} & \multirow[b]{2}{*}{$\begin{array}{l}\text { Casing } \\
\text { depth } \\
\text { (feet) }\end{array}$} & \multirow{2}{*}{$\begin{array}{l}\text { Depth } \\
\text { to } \\
\text { bedrock } \\
\text { (feet) }\end{array}$} & \multirow[b]{2}{*}{$\begin{array}{c}\text { Geologic } \\
\text { unit }\end{array}$} \\
\hline & $\begin{array}{l}\text { Lati- } \\
\text { tude } \\
\ldots \text {. }\end{array}$ & $\begin{array}{c}\text { Longi- } \\
\text { tude } \\
\ldots \text {.. }\end{array}$ & $\begin{array}{l}\text { Accu- } \\
\text { racy }\end{array}$ & & & & & & & \\
\hline $\begin{array}{l}571 \\
572 \\
573 \\
574 \\
575\end{array}$ & $\begin{array}{l}422842 \\
422843 \\
422844 \\
422844 \\
422846\end{array}$ & $\begin{array}{l}0753025 \\
0753006 \\
0753957 \\
0754507 \\
0753213\end{array}$ & $\begin{array}{l}\mathbf{T} \\
\mathbf{T} \\
\mathbf{T} \\
\mathbf{T} \\
\mathbf{T}\end{array}$ & $\begin{array}{l}\text { Ackerman } \\
\text { Macduff } \\
\text { Morley } \\
\text { Heckertt } \\
\text { Chenango Asphalt }\end{array}$ & $\begin{array}{l}12-11-87 \\
04-03-85 \\
06-05-86 \\
07-25-86 \\
09-29-88\end{array}$ & $\begin{array}{l}1295 \\
1270 \\
1510 \\
1280 \\
1010\end{array}$ & $\begin{array}{l}198 \\
155 \\
242 \\
150 \\
230\end{array}$ & $\begin{array}{r}42 \\
15 \\
230 \\
31 \\
230\end{array}$ & $\begin{array}{r}42 \\
15 \\
230 \\
31 \\
--\end{array}$ & $\begin{array}{l}\text { BDRK } \\
\text { BDRK } \\
\text { BDRK } \\
\text { BDRK } \\
\text { QRNR }\end{array}$ \\
\hline $\begin{array}{l}576 \\
577 \\
578 \\
579 \\
581\end{array}$ & $\begin{array}{l}422849 \\
422850 \\
422850 \\
422851 \\
422911\end{array}$ & $\begin{array}{l}0754703 \\
0753408 \\
0754950 \\
0753410 \\
0754011\end{array}$ & $\begin{array}{l}\mathbf{T} \\
\mathbf{T} \\
\mathbf{T} \\
\mathbf{T} \\
\mathbf{T}\end{array}$ & $\begin{array}{l}\text { Thau } \\
\text { Young } \\
\text { Shlag } \\
\text { Wakefield } \\
\text { Copeland }\end{array}$ & $\begin{array}{c}10-03-88 \\
03-01-85 \\
-- \\
02-19-85 \\
--\end{array}$ & $\begin{array}{l}1585 \\
1220 \\
1470 \\
1240 \\
1560\end{array}$ & $\begin{array}{r}73 \\
250 \\
200 \\
150 \\
214\end{array}$ & $\begin{array}{r}21 \\
84 \\
170 \\
75 \\
214\end{array}$ & $\begin{array}{r}21 \\
84 \\
170 \\
75 \\
--\end{array}$ & $\begin{array}{l}\text { BDRK } \\
\text { BDRK } \\
\text { BDRK } \\
\text { BDRK } \\
\text { QRNR }\end{array}$ \\
\hline $\begin{array}{l}582 \\
583 \\
584 \\
585 \\
586\end{array}$ & $\begin{array}{l}422912 \\
422922 \\
422924 \\
422925 \\
422932\end{array}$ & $\begin{array}{l}0755041 \\
0752939 \\
0754834 \\
0752940 \\
0753203\end{array}$ & $\begin{array}{l}\mathbf{T} \\
\mathbf{F} \\
\mathbf{T} \\
\mathbf{T} \\
\mathbf{T}\end{array}$ & $\begin{array}{l}\text { Warner } \\
\text { St. John } \\
\text { Kerns } \\
\text { Ewen } \\
\text { Huff }\end{array}$ & $\begin{array}{c}-- \\
-- \\
10-07-88 \\
04-02-87\end{array}$ & $\begin{array}{r}1520 \\
1520 \\
1450 \\
1520 \\
995\end{array}$ & $\begin{array}{l}196 \\
379 \\
123 \\
213 \\
223\end{array}$ & $\begin{array}{l}12 \\
74 \\
93 \\
80 \\
80\end{array}$ & $\begin{array}{l}12 \\
74 \\
93 \\
80 \\
80\end{array}$ & $\begin{array}{l}\text { BDRK } \\
\text { BDRK } \\
\text { BDRK } \\
\text { BDRK } \\
\text { BDRK }\end{array}$ \\
\hline $\begin{array}{l}587 \\
588 \\
589 \\
590 \\
591\end{array}$ & $\begin{array}{l}422933 \\
422943 \\
422944 \\
422946 \\
422948\end{array}$ & $\begin{array}{l}0755138 \\
0754103 \\
0754047 \\
0754611 \\
0754605\end{array}$ & $\begin{array}{l}\mathbf{T} \\
\mathbf{T} \\
\mathbf{T} \\
\mathbf{T} \\
\mathbf{T}\end{array}$ & $\begin{array}{l}\text { Handwerker } \\
\text { Redenbeck } \\
\text { Martin } \\
\text { Sayles } \\
\text { Morley }\end{array}$ & $\begin{array}{c}-- \\
06-26-85 \\
08-22-85 \\
09-09-87 \\
-\end{array}$ & $\begin{array}{l}1625 \\
1550 \\
1500 \\
1410 \\
1420\end{array}$ & $\begin{array}{r}150 \\
235 \\
247 \\
38 \\
165\end{array}$ & $\begin{array}{r}31 \\
84 \\
192 \\
-\overline{44}\end{array}$ & $\begin{array}{r}31 \\
84 \\
192 \\
\ddot{44}\end{array}$ & \begin{tabular}{l} 
BDRK \\
BDRK \\
BDRK \\
\hdashline$\quad$ \\
BDRK
\end{tabular} \\
\hline $\begin{array}{l}592 \\
593 \\
594 \\
595 \\
596\end{array}$ & $\begin{array}{l}422953 \\
422953 \\
422953 \\
422954 \\
422955\end{array}$ & $\begin{array}{l}0754521 \\
0754605 \\
0754611 \\
0754525 \\
0754611\end{array}$ & $\begin{array}{l}T \\
T \\
T \\
T \\
T\end{array}$ & $\begin{array}{l}\text { Brewer } \\
\text { Deuel } \\
\text { Beckwith } \\
\text { Wall } \\
\text { Chen. Rehab. Prog. }\end{array}$ & $\begin{array}{c}-\ddot{02-87} \\
\ddot{0} \\
03-24-87 \\
06-06-88\end{array}$ & $\begin{array}{l}1325 \\
1415 \\
1420 \\
1320 \\
1430\end{array}$ & $\begin{array}{r}248 \\
73 \\
130 \\
218 \\
52\end{array}$ & $\begin{array}{r}174 \\
23 \\
18 \\
185 \\
15\end{array}$ & $\begin{array}{r}174 \\
23 \\
18 \\
185 \\
15\end{array}$ & $\begin{array}{l}\text { BDRK } \\
\text { BDRK } \\
\text { BDRK } \\
\text { BDRK } \\
\text { BDRK }\end{array}$ \\
\hline $\begin{array}{l}597 \\
598 \\
599 \\
600 \\
601\end{array}$ & $\begin{array}{l}423013 \\
423013 \\
423015 \\
423015 \\
423023\end{array}$ & $\begin{array}{l}0752940 \\
0754130 \\
0754138 \\
0754504 \\
0753039\end{array}$ & $\begin{array}{l}\mathbf{T} \\
\mathbf{T} \\
\mathbf{T} \\
\mathbf{T} \\
\mathbf{T}\end{array}$ & $\begin{array}{l}\text { Leahy } \\
\text { Ingraham } \\
\text { Bradt } \\
\text { Wilson } \\
\text { Hayes }\end{array}$ & $\begin{array}{l}05-29-86 \\
09-15-86 \\
05-14-86 \\
05-13-88 \\
08-20-85\end{array}$ & $\begin{array}{l}1540 \\
1575 \\
1600 \\
1275 \\
1005\end{array}$ & $\begin{array}{l}250 \\
131 \\
150 \\
397 \\
105\end{array}$ & $\begin{array}{l}80 \\
10 \\
25 \\
99 \\
68\end{array}$ & $\begin{array}{l}80 \\
10 \\
25 \\
99 \\
68\end{array}$ & $\begin{array}{l}\text { BDRK } \\
\text { BDRK } \\
\text { BDRK } \\
\text { BDRK } \\
\text { BDRK }\end{array}$ \\
\hline $\begin{array}{l}602 \\
603 \\
604 \\
606 \\
607\end{array}$ & $\begin{array}{l}423025 \\
423028 \\
423028 \\
423043 \\
423047\end{array}$ & $\begin{array}{l}0753031 \\
0753035 \\
0754751 \\
0755147 \\
0752938\end{array}$ & $\begin{array}{l}\mathbf{T} \\
\mathbf{T} \\
\mathbf{T} \\
\mathbf{T} \\
\mathbf{T}\end{array}$ & $\begin{array}{l}\text { Johnson } \\
\text { Thompson } \\
\text { Fink } \\
\text { Kerns } \\
\text { Mills }\end{array}$ & $\begin{array}{c}03-20-85 \\
12-20-88 \\
-- \\
-- \\
-\end{array}$ & $\begin{array}{l}1080 \\
1020 \\
1590 \\
1550 \\
1130\end{array}$ & $\begin{array}{r}400 \\
247 \\
224 \\
116 \\
96\end{array}$ & $\begin{array}{l}49 \\
91 \\
48 \\
44 \\
20\end{array}$ & $\begin{array}{l}49 \\
91 \\
48 \\
44 \\
20\end{array}$ & $\begin{array}{l}\text { BDRK } \\
\text { BDRK } \\
\text { BDRK } \\
\text { BDRK } \\
\text { BDRK }\end{array}$ \\
\hline $\begin{array}{l}608 \\
609 \\
610 \\
611 \\
612\end{array}$ & $\begin{array}{l}423049 \\
423051 \\
423101 \\
423108 \\
423108\end{array}$ & $\begin{array}{l}0752925 \\
0752404 \\
0753931 \\
0752841 \\
0754457\end{array}$ & $\begin{array}{l}\mathbf{T} \\
\mathbf{T} \\
\mathbf{T} \\
\mathbf{T} \\
\mathbf{T}\end{array}$ & $\begin{array}{l}\text { Vidler } \\
\text { Holmesville Bapt. Ch. } \\
\text { Blivin } \\
\text { Slive } \\
\text { Mohin }\end{array}$ & $\begin{array}{c}08-06-87 \\
-- \\
-- \\
-- \\
--\end{array}$ & $\begin{array}{l}1160 \\
1055 \\
1620 \\
1330 \\
1485\end{array}$ & $\begin{array}{l}123 \\
170 \\
151 \\
135 \\
124\end{array}$ & $\begin{array}{r}35 \\
120 \\
37 \\
85 \\
32\end{array}$ & $\begin{array}{r}35 \\
120 \\
37 \\
85 \\
32\end{array}$ & $\begin{array}{l}\text { BDRK } \\
\text { BDRK } \\
\text { BDRK } \\
\text { BDRK } \\
\text { BDRK }\end{array}$ \\
\hline $\begin{array}{l}613 \\
614 \\
615 \\
616 \\
617\end{array}$ & $\begin{array}{l}423110 \\
423116 \\
423116 \\
423122 \\
423133\end{array}$ & $\begin{array}{l}0752812 \\
0753326 \\
0754412 \\
0754003 \\
0754530\end{array}$ & $\begin{array}{l}\mathbf{T} \\
\mathbf{T} \\
\mathbf{T} \\
\mathbf{T} \\
\mathbf{T}\end{array}$ & $\begin{array}{l}\text { Hill } \\
\text { Manley } \\
\text { Kasmarcik } \\
\text { Bliven } \\
\text { Mead }\end{array}$ & $\begin{array}{c}02-08-87 \\
-- \\
-- \\
-- \\
--\end{array}$ & $\begin{array}{l}1460 \\
1270 \\
1370 \\
1640 \\
1745\end{array}$ & $\begin{array}{r}212 \\
202 \\
133 \\
69 \\
166\end{array}$ & $\begin{array}{r}86 \\
14 \\
133 \\
5 \\
13\end{array}$ & $\begin{array}{r}86 \\
14 \\
-\ddot{5} \\
13\end{array}$ & $\begin{array}{l}\text { BDRK } \\
\text { BDRK } \\
\text { QRNR } \\
\text { BDRK } \\
\text { BDRK }\end{array}$ \\
\hline $\begin{array}{l}618 \\
619 \\
620 \\
621 \\
622\end{array}$ & $\begin{array}{l}423137 \\
423143 \\
423147 \\
423149 \\
423154\end{array}$ & $\begin{array}{l}0752817 \\
0754520 \\
0753206 \\
0754525 \\
0752852\end{array}$ & $\begin{array}{l}\mathbf{T} \\
\mathbf{T} \\
\mathbf{T} \\
\mathbf{T} \\
\mathbf{T}\end{array}$ & $\begin{array}{l}\text { Miller } \\
\text { Clifford } \\
\text { Libby } \\
\text { Piccione } \\
\text { Sidari }\end{array}$ & $\begin{array}{c}07-11-85 \\
05-25-86 \\
08-08-85 \\
-- \\
10-06-86\end{array}$ & $\begin{array}{l}1410 \\
1665 \\
1160 \\
1655 \\
1645\end{array}$ & $\begin{array}{l}150 \\
150 \\
300 \\
250 \\
373\end{array}$ & $\begin{array}{l}53 \\
30 \\
30 \\
26 \\
85\end{array}$ & $\begin{array}{l}53 \\
30 \\
30 \\
26 \\
85\end{array}$ & $\begin{array}{l}\text { BDRK } \\
\text { BDRK } \\
\text { BDRK } \\
\text { BDRK } \\
\text { BDRK }\end{array}$ \\
\hline
\end{tabular}


Table 1.--Records of wells and test holes--continued.

[Dashes indicate no data. Locations shown on pls. 1A, 1B.]




Table 1.--Records of wells and test holes--continued.

[Dashes indicate no data. Locations shown on pls. 1A, 1B.]

\begin{tabular}{|c|c|c|c|c|c|c|c|c|c|c|}
\hline $\begin{array}{c}\text { Well } \\
\text { number }\end{array}$ & $\begin{array}{l}\text { Lati- } \\
\text { tude } \\
\ldots .\end{array}$ & $\begin{array}{c}\text { Location } \\
\text { Longi- } \\
\text { tude } \\
\text {... }\end{array}$ & $\begin{array}{c}\text { Accu- } \\
\text { racy }\end{array}$ & Owner & $\begin{array}{c}\text { Date } \\
\text { drilled }\end{array}$ & $\begin{array}{c}\text { Altitude } \\
\text { of land } \\
\text { surface } \\
\text { (feet) }\end{array}$ & $\begin{array}{l}\text { Well } \\
\text { depth } \\
\text { (feet) }\end{array}$ & $\begin{array}{l}\text { Casing } \\
\text { depth } \\
\text { (feet) }\end{array}$ & $\begin{array}{l}\text { Depth } \\
\text { to } \\
\text { bedrock } \\
\text { (feet) }\end{array}$ & $\begin{array}{c}\text { Geologic } \\
\text { unit }\end{array}$ \\
\hline $\begin{array}{l}623 \\
624 \\
625 \\
626 \\
627\end{array}$ & $\begin{array}{l}423158 \\
423201 \\
423208 \\
423222 \\
423230\end{array}$ & $\begin{array}{l}0753212 \\
0754310 \\
0753722 \\
0753325 \\
0753027\end{array}$ & $\begin{array}{l}\mathrm{T} \\
\mathrm{T} \\
\mathrm{T} \\
\mathrm{T} \\
\mathrm{T}\end{array}$ & $\begin{array}{l}\text { Barrowclough } \\
\text { Kent } \\
\text { Coleman } \\
\text { Abbott } \\
\text { Fama }\end{array}$ & $\begin{array}{c}08-03-85 \\
-- \\
08-19-88 \\
-- \\
--\end{array}$ & $\begin{array}{l}1140 \\
1630 \\
1510 \\
1480 \\
1030\end{array}$ & $\begin{array}{l}300 \\
176 \\
175 \\
106 \\
377\end{array}$ & $\begin{array}{l}14 \\
92 \\
54 \\
20 \\
47\end{array}$ & $\begin{array}{l}14 \\
92 \\
54 \\
20 \\
47\end{array}$ & $\begin{array}{l}\text { BDRK } \\
\text { BDRK } \\
\text { BDRK } \\
\text { BDRK } \\
\text { BDRK }\end{array}$ \\
\hline $\begin{array}{l}628 \\
630 \\
632 \\
633 \\
634\end{array}$ & $\begin{array}{l}423230 \\
423253 \\
423308 \\
423318 \\
423319\end{array}$ & $\begin{array}{l}0753042 \\
0754401 \\
0752859 \\
0754303 \\
0752909\end{array}$ & $\begin{array}{l}\mathrm{T} \\
\mathrm{T} \\
\mathrm{T} \\
\mathrm{T} \\
\mathrm{T}\end{array}$ & $\begin{array}{l}\text { Barrett } \\
\text { Petrizzo } \\
\text { Todd } \\
\text { Grover } \\
\text { Jankowski }\end{array}$ & $\begin{array}{c}\text { 11- } \overline{13-87} \\
-- \\
06-02-88 \\
--\end{array}$ & $\begin{array}{l}1010 \\
1590 \\
1290 \\
1545 \\
1480\end{array}$ & $\begin{array}{l}257 \\
125 \\
150 \\
222 \\
221\end{array}$ & $\begin{array}{r}257 \\
62 \\
43 \\
100 \\
40\end{array}$ & $\begin{array}{r}-- \\
62 \\
43 \\
100 \\
40\end{array}$ & $\begin{array}{l}\text { QRNR } \\
\text { BDRK } \\
\text { BDRK } \\
\text { BDRK } \\
\text { BDRK }\end{array}$ \\
\hline $\begin{array}{l}635 \\
636 \\
637 \\
638 \\
639\end{array}$ & $\begin{array}{l}423321 \\
423321 \\
423322 \\
423325 \\
423325\end{array}$ & $\begin{array}{l}0752154 \\
0752859 \\
0753944 \\
0753253 \\
0753253\end{array}$ & $\begin{array}{l}T \\
T \\
T \\
T \\
T\end{array}$ & $\begin{array}{l}\text { Hearth } \\
\text { Ellis } \\
\text { McConagie } \\
\text { Serafen } \\
\text { Serafen }\end{array}$ & $\begin{array}{c}-- \\
-- \\
04-04-86 \\
11-01-85 \\
--\end{array}$ & $\begin{array}{l}1075 \\
1510 \\
1685 \\
1175 \\
1175\end{array}$ & $\begin{array}{l}190 \\
154 \\
197 \\
170 \\
148\end{array}$ & $\begin{array}{r}153 \\
14 \\
41 \\
78 \\
86\end{array}$ & $\begin{array}{r}153 \\
14 \\
41 \\
78 \\
86\end{array}$ & $\begin{array}{l}\text { BDRK } \\
\text { BDRK } \\
\text { BDRK } \\
\text { BDRK } \\
\text { BDRK }\end{array}$ \\
\hline $\begin{array}{l}641 \\
642 \\
643 \\
644 \\
645\end{array}$ & $\begin{array}{l}423328 \\
423336 \\
423337 \\
423341 \\
423345\end{array}$ & $\begin{array}{l}0754333 \\
0753726 \\
0752843 \\
0752838 \\
0753729\end{array}$ & $\begin{array}{l}\mathrm{T} \\
\mathrm{T} \\
\mathrm{T} \\
\mathrm{T} \\
\mathrm{T}\end{array}$ & $\begin{array}{l}\text { Viola } \\
\text { Berkeley } \\
\text { Brush } \\
\text { Tamsett } \\
\text { Conant }\end{array}$ & $\begin{array}{l}05-16-86 \\
09-02-86 \\
10-02-86 \\
04-17-86 \\
03-30-88\end{array}$ & $\begin{array}{l}1750 \\
1725 \\
1490 \\
1470 \\
1800\end{array}$ & $\begin{array}{l}197 \\
173 \\
198 \\
225 \\
247\end{array}$ & $\begin{array}{l}65 \\
21 \\
31 \\
63 \\
90\end{array}$ & $\begin{array}{l}65 \\
21 \\
31 \\
63 \\
90\end{array}$ & $\begin{array}{l}\text { BDRK } \\
\text { BDRK } \\
\text { BDRK } \\
\text { BDRK } \\
\text { BDRK }\end{array}$ \\
\hline $\begin{array}{l}646 \\
647 \\
648 \\
649 \\
650\end{array}$ & $\begin{array}{l}423349 \\
423355 \\
423401 \\
423409 \\
423411\end{array}$ & $\begin{array}{l}0753332 \\
0753313 \\
0753646 \\
0753655 \\
0752720\end{array}$ & $\begin{array}{l}\mathrm{T} \\
\mathrm{T} \\
\mathrm{T} \\
\mathrm{T} \\
\mathrm{T}\end{array}$ & $\begin{array}{l}\text { Bush } \\
\text { Steiber } \\
\text { Blackman } \\
\text { Towner } \\
\text { Portluck }\end{array}$ & $\begin{array}{c}06-27-87 \\
-- \\
06-30-87 \\
06-02-86 \\
--\end{array}$ & $\begin{array}{l}1310 \\
1180 \\
1785 \\
1795 \\
1840\end{array}$ & $\begin{array}{l}210 \\
379 \\
150 \\
248 \\
226\end{array}$ & $\begin{array}{l}70 \\
74 \\
90 \\
84 \\
10\end{array}$ & $\begin{array}{l}70 \\
74 \\
90 \\
84 \\
10\end{array}$ & $\begin{array}{l}\text { BDRK } \\
\text { BDRK } \\
\text { BDRK } \\
\text { BDRK } \\
\text { BDRK }\end{array}$ \\
\hline $\begin{array}{l}651 \\
652 \\
653 \\
654 \\
655\end{array}$ & $\begin{array}{l}423411 \\
423418 \\
423419 \\
423428 \\
423428\end{array}$ & $\begin{array}{l}0752728 \\
0753150 \\
0752747 \\
0752605 \\
0753455\end{array}$ & $\begin{array}{l}\mathrm{T} \\
\mathrm{T} \\
\mathrm{T} \\
\mathrm{T} \\
\mathrm{T}\end{array}$ & $\begin{array}{l}\text { Cloyd } \\
\text { Cordaro } \\
\text { Pratt } \\
\text { Beadle } \\
\text { Debello }\end{array}$ & $\begin{array}{c}09-17-85 \\
07-02-88 \\
-- \\
09-19-85 \\
08-05-88\end{array}$ & $\begin{array}{l}1820 \\
1090 \\
1670 \\
1750 \\
1460\end{array}$ & $\begin{array}{l}172 \\
497 \\
175 \\
247 \\
198\end{array}$ & $\begin{array}{r}26 \\
105 \\
134 \\
170 \\
73\end{array}$ & $\begin{array}{r}26 \\
105 \\
134 \\
170 \\
73\end{array}$ & $\begin{array}{l}\text { BDRK } \\
\text { BDRK } \\
\text { BDRK } \\
\text { BDRK } \\
\text { BDRK }\end{array}$ \\
\hline $\begin{array}{l}656 \\
657 \\
658 \\
659 \\
660\end{array}$ & $\begin{array}{l}423428 \\
423432 \\
423436 \\
423438 \\
423443\end{array}$ & $\begin{array}{l}0753459 \\
0753524 \\
0752512 \\
0752409 \\
0752019\end{array}$ & $\begin{array}{l}\mathrm{T} \\
\mathrm{T} \\
\mathrm{T} \\
\mathrm{T} \\
\mathrm{T}\end{array}$ & $\begin{array}{l}\text { Affuso } \\
\text { Conant } \\
\text { Greenberg } \\
\text { Brooks } \\
\text { Parks }\end{array}$ & $\begin{array}{l}11-27-87 \\
11-25-87 \\
09-04-86 \\
07-08-88 \\
11-14-86\end{array}$ & $\begin{array}{l}1470 \\
1605 \\
1600 \\
1360 \\
1080\end{array}$ & $\begin{array}{l}165 \\
172 \\
196 \\
101 \\
170\end{array}$ & $\begin{array}{r}84 \\
8 \\
21 \\
53 \\
155\end{array}$ & $\begin{array}{r}84 \\
8 \\
21 \\
53 \\
155\end{array}$ & $\begin{array}{l}\text { BDRK } \\
\text { BDRK } \\
\text { BDRK } \\
\text { BDRK } \\
\text { BDRK }\end{array}$ \\
\hline $\begin{array}{l}661 \\
662 \\
663 \\
664 \\
665\end{array}$ & $\begin{array}{l}423443 \\
423502 \\
423508 \\
423511 \\
423514\end{array}$ & $\begin{array}{l}0752639 \\
0754647 \\
0754620 \\
0753559 \\
0752603\end{array}$ & $\begin{array}{l}\mathrm{T} \\
\mathrm{T} \\
\mathrm{T} \\
\mathrm{T} \\
\mathrm{T}\end{array}$ & $\begin{array}{l}\text { Hunt } \\
\text { Peaslee } \\
\text { Jackson } \\
\text { Martini } \\
\text { Stockwell }\end{array}$ & $\begin{array}{l}06-18-88 \\
06-02-89 \\
05-12-88 \\
09-29-87 \\
04-03-87\end{array}$ & $\begin{array}{l}1760 \\
1585 \\
1575 \\
1580 \\
1770\end{array}$ & $\begin{array}{l}149 \\
240 \\
222 \\
223 \\
200\end{array}$ & $\begin{array}{l}86 \\
10 \\
48 \\
90 \\
10\end{array}$ & $\begin{array}{l}86 \\
10 \\
48 \\
90 \\
10\end{array}$ & $\begin{array}{l}\text { BDRK } \\
\text { BDRK } \\
\text { BDRK } \\
\text { BDRK } \\
\text { BDRK }\end{array}$ \\
\hline $\begin{array}{l}666 \\
667 \\
668 \\
670 \\
672\end{array}$ & $\begin{array}{l}423514 \\
423516 \\
423552 \\
423620 \\
423645\end{array}$ & $\begin{array}{l}0752744 \\
0752723 \\
0753601 \\
0754004 \\
0752705\end{array}$ & $\begin{array}{l}\mathrm{T} \\
\mathrm{T} \\
\mathrm{T} \\
\mathrm{T} \\
\mathrm{T}\end{array}$ & $\begin{array}{l}\text { Fessler } \\
\text { Flanagan } \\
\text { Stone } \\
\text { Whipple } \\
\text { Tomsic }\end{array}$ & $\begin{array}{l}08-09-85 \\
10-08-85 \\
11-01-88 \\
04-15-88 \\
07-24-89\end{array}$ & $\begin{array}{l}1530 \\
1620 \\
1225 \\
1510 \\
1580\end{array}$ & $\begin{array}{l}197 \\
400 \\
147 \\
198 \\
175\end{array}$ & $\begin{array}{r}123 \\
140 \\
64 \\
20 \\
73\end{array}$ & $\begin{array}{r}123 \\
140 \\
64 \\
20 \\
73\end{array}$ & $\begin{array}{l}\text { BDRK } \\
\text { BDRK } \\
\text { BDRK } \\
\text { BDRK } \\
\text { BDRK }\end{array}$ \\
\hline $\begin{array}{l}673 \\
674 \\
675 \\
677 \\
678\end{array}$ & $\begin{array}{l}423652 \\
423654 \\
423703 \\
423709 \\
423717\end{array}$ & $\begin{array}{l}0753131 \\
0753127 \\
0752751 \\
0752934 \\
0752845\end{array}$ & $\begin{array}{l}\mathrm{T} \\
\mathrm{T} \\
\mathrm{T} \\
\mathrm{T} \\
\mathrm{T}\end{array}$ & $\begin{array}{l}\text { Camenga } \\
\text { McNulty } \\
\text { Willis } \\
\text { Macedonio } \\
\text { Eggers }\end{array}$ & $\begin{array}{l}09-25-88 \\
11-04-86 \\
09-16-86 \\
04-17-87 \\
07-24-87\end{array}$ & $\begin{array}{l}1025 \\
1020 \\
1765 \\
1625 \\
1770\end{array}$ & $\begin{array}{r}23 \\
397 \\
120 \\
338 \\
250\end{array}$ & $\begin{array}{r}23 \\
397 \\
35 \\
24 \\
50\end{array}$ & $\begin{array}{l}-- \\
-- \\
35 \\
24 \\
50\end{array}$ & $\begin{array}{l}\text { QRNR } \\
\text { QRNR } \\
\text { BDRK } \\
\text { BDRK } \\
\text { BDRK }\end{array}$ \\
\hline
\end{tabular}


Table 1.--Records of wells and test holes--continued. [Dashes indicate no data. Locations shown on pls. 1A, 1B.]

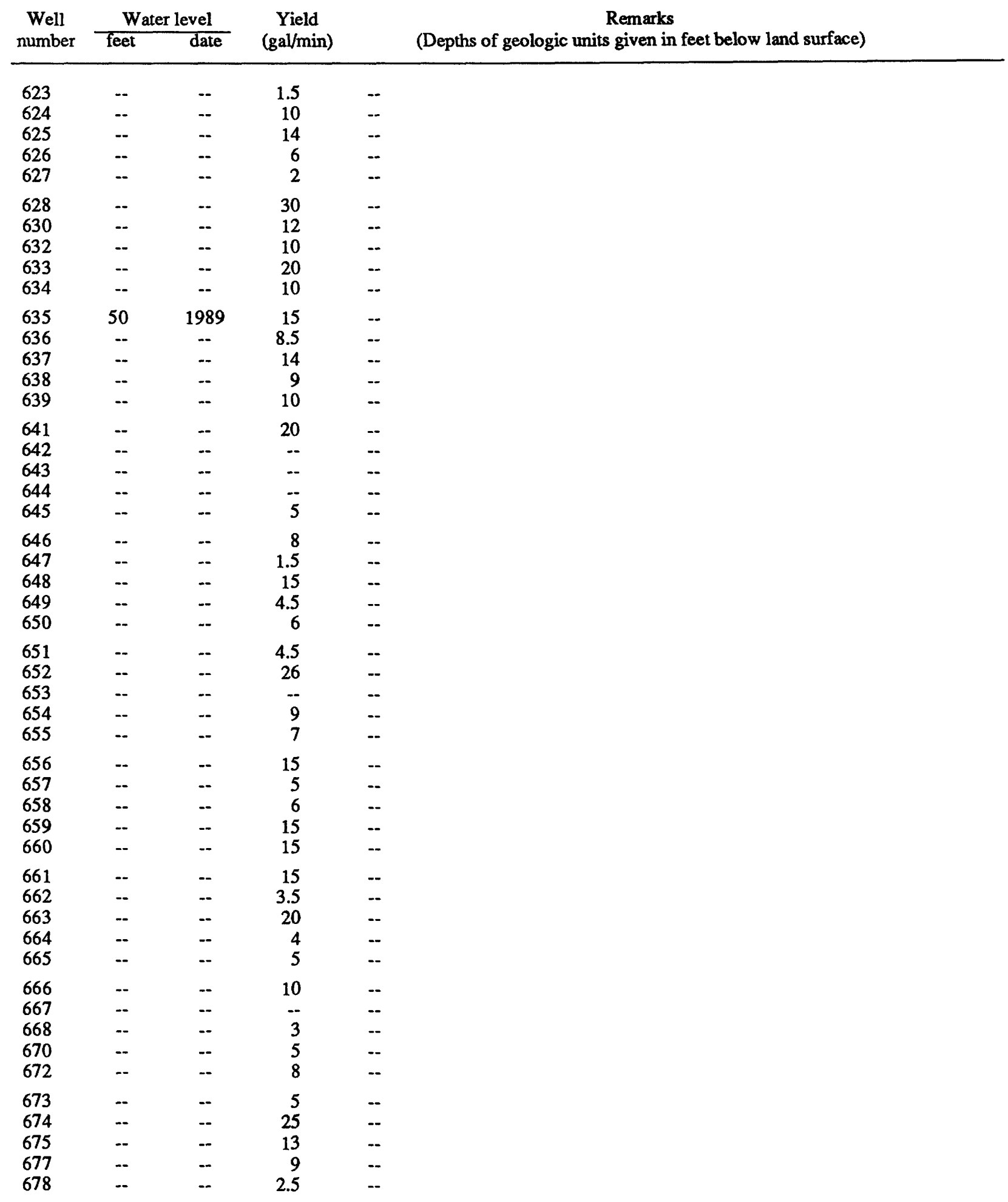


Table 1.--Records of wells and test holes--continued.

[Dashes indicate no data. Locations shown on pls. 1A, 1B.]

\begin{tabular}{|c|c|c|c|c|c|c|c|c|c|c|}
\hline $\begin{array}{c}\text { Well } \\
\text { number }\end{array}$ & $\begin{array}{l}\text { Lati- } \\
\text { tude } \\
\cdots \cdots\end{array}$ & $\begin{array}{c}\text { Location } \\
\text { Longi- } \\
\text { tude } \\
\text {.. }\end{array}$ & $\begin{array}{l}\text { Accu- } \\
\text { racy }\end{array}$ & Owner & $\begin{array}{c}\text { Date } \\
\text { drilled }\end{array}$ & $\begin{array}{c}\text { Altitude } \\
\text { of land } \\
\text { surface } \\
\text { (feet) }\end{array}$ & $\begin{array}{l}\text { Well } \\
\text { depth } \\
\text { (feet) }\end{array}$ & $\begin{array}{l}\text { Casing } \\
\text { depth } \\
\text { (feet) }\end{array}$ & $\begin{array}{l}\text { Depth } \\
\text { to } \\
\text { bedrock } \\
\text { (feet) }\end{array}$ & $\begin{array}{c}\text { Geologic } \\
\text { unit }\end{array}$ \\
\hline $\begin{array}{l}679 \\
680 \\
681 \\
682 \\
683\end{array}$ & $\begin{array}{l}423740 \\
423742 \\
423803 \\
423807 \\
423808\end{array}$ & $\begin{array}{l}0752743 \\
0751959 \\
0752919 \\
0752838 \\
0753928\end{array}$ & $\begin{array}{l}\mathbf{T} \\
\mathbf{T} \\
\mathbf{T} \\
\mathbf{T} \\
\mathbf{T}\end{array}$ & $\begin{array}{l}\text { McNulty } \\
\text { Johnson } \\
\text { Walker } \\
\text { Campbell } \\
\text { Smith }\end{array}$ & $\begin{array}{c}-- \\
07-\overline{01-84} \\
\overline{10-03-85}\end{array}$ & $\begin{array}{l}1400 \\
1120 \\
1100 \\
1250 \\
1860\end{array}$ & $\begin{array}{r}21 \\
310 \\
65 \\
150 \\
173\end{array}$ & $\begin{array}{l}16 \\
52 \\
15 \\
52 \\
74\end{array}$ & $\begin{array}{l}16 \\
52 \\
15 \\
52 \\
74\end{array}$ & $\begin{array}{l}\text { BDRK } \\
\text { BDRK } \\
\text { BDRK } \\
\text { BDRK } \\
\text { BDRK }\end{array}$ \\
\hline $\begin{array}{l}684 \\
685 \\
686 \\
687 \\
688\end{array}$ & $\begin{array}{l}423810 \\
423841 \\
423842 \\
423855 \\
423901\end{array}$ & $\begin{array}{l}0752854 \\
0752325 \\
0751947 \\
0753246 \\
0753447\end{array}$ & $\begin{array}{l}\mathrm{T} \\
\mathrm{T} \\
\mathrm{T} \\
\mathrm{T} \\
\mathrm{T}\end{array}$ & $\begin{array}{l}\text { Buckley } \\
\text { Bochen } \\
\text { Van Dyke } \\
\text { Onyan } \\
\text { Hicks }\end{array}$ & $\begin{array}{c}06-28-88 \\
03-29-85 \\
11-01-85 \\
-- \\
10-20-84\end{array}$ & $\begin{array}{l}1240 \\
1590 \\
1115 \\
1090 \\
1470\end{array}$ & $\begin{array}{r}458 \\
225 \\
135 \\
81 \\
100\end{array}$ & $\begin{array}{r}52 \\
23 \\
135 \\
76 \\
20\end{array}$ & $\begin{array}{r}52 \\
23 \\
-- \\
76 \\
20\end{array}$ & $\begin{array}{l}\text { BDRK } \\
\text { BDRK } \\
\text { QRNR } \\
\text { BDRK } \\
\text { BDRK }\end{array}$ \\
\hline $\begin{array}{l}689 \\
690 \\
691 \\
692 \\
693\end{array}$ & $\begin{array}{l}423902 \\
423912 \\
423916 \\
423917 \\
423919\end{array}$ & $\begin{array}{l}0753911 \\
0753727 \\
0753749 \\
0753902 \\
0752806\end{array}$ & $\begin{array}{l}\mathbf{T} \\
\mathbf{T} \\
\mathbf{T} \\
\mathbf{T} \\
\mathbf{T}\end{array}$ & $\begin{array}{l}\text { Thompson } \\
\text { Anderson } \\
\text { Furey } \\
\text { Nichols } \\
\text { McNeil }\end{array}$ & $\begin{array}{c}05-01-86 \\
03-22-86 \\
03-22-89 \\
09-08-86 \\
--\end{array}$ & $\begin{array}{l}1375 \\
1400 \\
1640 \\
1460 \\
1330\end{array}$ & $\begin{array}{r}85 \\
223 \\
210 \\
32 \\
174\end{array}$ & $\begin{array}{r}85 \\
120 \\
10 \\
32 \\
46\end{array}$ & $\begin{array}{r}-- \\
120 \\
10 \\
-- \\
46\end{array}$ & $\begin{array}{l}\text { QRNR } \\
\text { BDRK } \\
\text { BDRK } \\
\text { QRNR } \\
\text { BDRK }\end{array}$ \\
\hline $\begin{array}{l}694 \\
695 \\
696 \\
697 \\
698\end{array}$ & $\begin{array}{l}423920 \\
423921 \\
423923 \\
423923 \\
423930\end{array}$ & $\begin{array}{l}0752830 \\
0753231 \\
0752721 \\
0752914 \\
0752956\end{array}$ & $\begin{array}{l}\mathbf{T} \\
\mathbf{T} \\
\mathbf{T} \\
\mathbf{T} \\
\mathbf{T}\end{array}$ & $\begin{array}{l}\text { Hendrickson } \\
\text { Pinney } \\
\text { Campbell } \\
\text { Ray } \\
\text { Lewis }\end{array}$ & $\begin{array}{l}06-18-86 \\
03-17-89 \\
09-24-87 \\
05-01-85 \\
03-25-86\end{array}$ & $\begin{array}{l}1220 \\
1250 \\
1350 \\
1165 \\
1190\end{array}$ & $\begin{array}{r}210 \\
358 \\
95 \\
180 \\
350\end{array}$ & $\begin{array}{r}35 \\
21 \\
95 \\
180 \\
85\end{array}$ & $\begin{array}{r}35 \\
21 \\
-- \\
-- \\
85\end{array}$ & $\begin{array}{l}\text { BDRK } \\
\text { BDRK } \\
\text { QRNR } \\
\text { QRNR } \\
\text { BDRK }\end{array}$ \\
\hline $\begin{array}{l}699 \\
700 \\
701 \\
702 \\
703\end{array}$ & $\begin{array}{l}423937 \\
423941 \\
424004 \\
424008 \\
424023\end{array}$ & $\begin{array}{l}0752912 \\
0752322 \\
0752640 \\
0752252 \\
0752156\end{array}$ & $\begin{array}{l}\mathrm{T} \\
\mathrm{T} \\
\mathrm{T} \\
\mathrm{T} \\
\mathrm{T}\end{array}$ & $\begin{array}{l}\text { Lacroce } \\
\text { Mcenroe } \\
\text { Joyce } \\
\text { Weiss } \\
\text { Loomis }\end{array}$ & $\begin{array}{l}10-08-87 \\
09-08-86 \\
02-25-85 \\
03-11-87 \\
06-02-86\end{array}$ & $\begin{array}{l}1055 \\
1550 \\
1570 \\
1375 \\
1270\end{array}$ & $\begin{array}{l}100 \\
225 \\
220 \\
320 \\
173\end{array}$ & $\begin{array}{l}43 \\
52 \\
57 \\
50 \\
70\end{array}$ & $\begin{array}{l}43 \\
52 \\
57 \\
50 \\
70\end{array}$ & $\begin{array}{l}\text { BDRK } \\
\text { BDRK } \\
\text { BDRK } \\
\text { BDRK } \\
\text { BDRK }\end{array}$ \\
\hline $\begin{array}{l}704 \\
705 \\
706 \\
707 \\
708\end{array}$ & $\begin{array}{l}424023 \\
424026 \\
424028 \\
424034 \\
424040\end{array}$ & $\begin{array}{l}0752908 \\
0752200 \\
0752950 \\
0752942 \\
0752959\end{array}$ & $\begin{array}{l}\mathrm{T} \\
\mathrm{T} \\
\mathrm{T} \\
\mathrm{T} \\
\mathrm{T}\end{array}$ & $\begin{array}{l}\text { Green } \\
\text { Muller } \\
\text { Van Wagner } \\
\text { Fuller } \\
\text { Kenyon Press }\end{array}$ & $\begin{array}{l}11-30-87 \\
05-25-89 \\
04-22-89 \\
08-13-86 \\
09-23-85\end{array}$ & $\begin{array}{l}1150 \\
1260 \\
1050 \\
1065 \\
1050\end{array}$ & $\begin{array}{r}298 \\
98 \\
175 \\
173 \\
220\end{array}$ & $\begin{array}{r}52 \\
67 \\
150 \\
66 \\
220\end{array}$ & $\begin{array}{r}52 \\
67 \\
150 \\
66 \\
--\end{array}$ & $\begin{array}{l}\text { BDRK } \\
\text { BDRK } \\
\text { BDRK } \\
\text { BDRK } \\
\text { QRNR }\end{array}$ \\
\hline $\begin{array}{l}709 \\
710 \\
711 \\
712 \\
713\end{array}$ & $\begin{array}{l}424042 \\
424048 \\
424049 \\
424057 \\
424058\end{array}$ & $\begin{array}{l}0752932 \\
0753725 \\
0752823 \\
0752059 \\
0752225\end{array}$ & $\begin{array}{l}\mathbf{T} \\
\mathbf{T} \\
\mathbf{T} \\
\mathbf{T} \\
\mathbf{T}\end{array}$ & $\begin{array}{l}\text { Bergeron } \\
\text { Ashcraft } \\
\text { Tredway } \\
\text { Winton } \\
\text { Dye }\end{array}$ & $\begin{array}{c}05-\overline{03-89} \\
-- \\
07-26-87 \\
06-01-86\end{array}$ & $\begin{array}{l}1075 \\
1830 \\
1300 \\
1250 \\
1290\end{array}$ & $\begin{array}{l}148 \\
351 \\
186 \\
123 \\
198\end{array}$ & $\begin{array}{l}83 \\
21 \\
20 \\
33 \\
84\end{array}$ & $\begin{array}{l}83 \\
21 \\
20 \\
33 \\
84\end{array}$ & $\begin{array}{l}\text { BDRK } \\
\text { BDRK } \\
\text { BDRK } \\
\text { BDRK } \\
\text { BDRK }\end{array}$ \\
\hline $\begin{array}{l}714 \\
715 \\
716 \\
717 \\
718\end{array}$ & $\begin{array}{l}424101 \\
424101 \\
424102 \\
424108 \\
424122\end{array}$ & $\begin{array}{l}0752222 \\
0752225 \\
0752219 \\
0752232 \\
0752015\end{array}$ & $\begin{array}{l}\mathbf{T} \\
\mathbf{T} \\
\mathbf{T} \\
\mathbf{T} \\
\mathbf{T}\end{array}$ & $\begin{array}{l}\text { Calhoun } \\
\text { Dippel } \\
\text { Furgison } \\
\text { Homann } \\
\text { Gay }\end{array}$ & $\begin{array}{l}01-07-87 \\
06-08-85 \\
06-23-86 \\
08-15-86 \\
07-16-88\end{array}$ & $\begin{array}{l}1300 \\
1295 \\
1300 \\
1360 \\
1320\end{array}$ & $\begin{array}{l}144 \\
150 \\
132 \\
135 \\
173\end{array}$ & $\begin{array}{l}21 \\
50 \\
26 \\
53 \\
33\end{array}$ & $\begin{array}{l}21 \\
50 \\
26 \\
53 \\
33\end{array}$ & $\begin{array}{l}\text { BDRK } \\
\text { BDRK } \\
\text { BDRK } \\
\text { BDRK } \\
\text { BDRK }\end{array}$ \\
\hline $\begin{array}{l}719 \\
720 \\
721 \\
722 \\
723\end{array}$ & $\begin{array}{l}424122 \\
424129 \\
424139 \\
424142 \\
424143\end{array}$ & $\begin{array}{l}0753621 \\
0752012 \\
0753027 \\
0753003 \\
0753030\end{array}$ & $\begin{array}{l}\mathbf{T} \\
\mathbf{T} \\
\mathbf{T} \\
\mathbf{T} \\
\mathbf{T}\end{array}$ & $\begin{array}{l}\text { Watkins } \\
\text { Woods } \\
\text { Baker } \\
\text { Fowlston } \\
\text { Bagnall }\end{array}$ & $\begin{array}{c}08-12-86 \\
08-24-88 \\
-- \\
09-21-87 \\
--\end{array}$ & $\begin{array}{l}1500 \\
1310 \\
1080 \\
1040 \\
1105\end{array}$ & $\begin{array}{l}175 \\
397 \\
198 \\
132 \\
198\end{array}$ & $\begin{array}{r}63 \\
21 \\
21 \\
132 \\
31\end{array}$ & $\begin{array}{r}63 \\
21 \\
21 \\
-- \\
31\end{array}$ & $\begin{array}{l}\text { BDRK } \\
\text { BDRK } \\
\text { BDRK } \\
\text { QRNR } \\
\text { BDRK }\end{array}$ \\
\hline $\begin{array}{l}724 \\
725 \\
726 \\
727 \\
728\end{array}$ & $\begin{array}{l}424147 \\
424159 \\
424201 \\
424220 \\
424221\end{array}$ & $\begin{array}{l}0753001 \\
0752459 \\
0752720 \\
0752727 \\
0752724\end{array}$ & $\begin{array}{l}\mathrm{T} \\
\mathrm{T} \\
\mathrm{T} \\
\mathrm{T} \\
\mathrm{T}\end{array}$ & $\begin{array}{l}\text { Boise } \\
\text { Hostman } \\
\text { Lane } \\
\text { Lachaplle } \\
\text { Wood }\end{array}$ & $\begin{array}{l}06-30-88 \\
03-12-86 \\
07-28-87 \\
06-13-88 \\
07-07-86\end{array}$ & $\begin{array}{l}1060 \\
1550 \\
1560 \\
1640 \\
1640\end{array}$ & $\begin{array}{r}46 \\
400 \\
350 \\
147 \\
126\end{array}$ & $\begin{array}{l}46 \\
21 \\
20 \\
32 \\
27\end{array}$ & $\begin{array}{l}-- \\
21 \\
20 \\
32 \\
27\end{array}$ & $\begin{array}{l}\text { QRNR } \\
\text { BDRK } \\
\text { BDRK } \\
\text { BDRK } \\
\text { BDRK }\end{array}$ \\
\hline
\end{tabular}


Table 1.--Records of wells and test holes--continued.

[Dashes indicate no data. Locations shown on pls. 1A, 1B.]

\begin{tabular}{|c|c|c|c|c|}
\hline \multirow{2}{*}{$\begin{array}{c}\text { Well } \\
\text { number }\end{array}$} & \multicolumn{2}{|c|}{ Water level } & \multirow{2}{*}{$\underset{(\mathrm{gal} / \mathrm{min})}{\text { Yield }}$} & \multirow{2}{*}{$\begin{array}{c}\text { Remarks } \\
\text { (Depths of geologic units given in feet below land surface) }\end{array}$} \\
\hline & feet & date & & \\
\hline 679 & -- & -- & 5 & -- \\
\hline 680 & -- & -- & 1.5 & -- \\
\hline 681 & 20 & $07-01-84$ & 25 & -- \\
\hline 682 & -- & -- & 10 & -- \\
\hline 683 & -- & -. & 15 & -- \\
\hline 684 & -- & -- & 3 & -- \\
\hline 685 & -- & -- & $\ldots$ & -- \\
\hline 686 & -- & -- & 24 & -- \\
\hline 687 & -- & -- & 9 & -- \\
\hline 688 & 50 & $10-20-84$ & 25 & -- \\
\hline 689 & -- & -- & 20 & -- \\
\hline 690 & -- & -- & 16 & -- \\
\hline 691 & -- & -- & 5 & -- \\
\hline 692 & -- & -- & 11 & -- \\
\hline 693 & -- & -- & 5 & - \\
\hline 694 & -- & -- & 4 & -- \\
\hline 695 & -- & -- & 1.5 & -- \\
\hline 696 & -- & -- & 5 & -- \\
\hline 697 & -- & -- & 12 & -- \\
\hline 698 & -- & -- & 15 & -- \\
\hline 699 & -- & -- & 9 & -- \\
\hline 700 & -- & -- & 7 & -- \\
\hline 701 & -- & -- & 3 & -- \\
\hline 702 & -- & -- & -- & -- \\
\hline 703 & -- & -- & -- & -- \\
\hline 704 & 200 & $11-30-87$ & 1.5 & - \\
\hline 705 & -- & -- & 25 & -- \\
\hline 706 & -- & -- & 18 & -- \\
\hline 707 & -- & -. & 8 & -- \\
\hline 708 & 3 & $09-23-85$ & 20 & - \\
\hline 709 & -- & -- & 10 & - \\
\hline 710 & -- & .- & 16 & -- \\
\hline 711 & -- & -- & 10 & -- \\
\hline 712 & -- & -- & 16 & -- \\
\hline 713 & -- & -- & -. & -- \\
\hline 714 & -- & -- & 30 & -- \\
\hline 715 & -- & -- & -- & -- \\
\hline 716 & 20 & $06-23-86$ & 15 & -.- \\
\hline 717 & -. & -- & -- & -- \\
\hline 718 & -- & -- & 5 & .- \\
\hline 719 & -- & -- & -. & .- \\
\hline 720 & -- & -- & 7 & -- \\
\hline 721 & -- & -- & 10 & .. \\
\hline 722 & -- & -- & 15 & -- \\
\hline 723 & -- & -- & 5 & -- \\
\hline 724 & -- & -- & 25 & -- \\
\hline 725 & -- & -- & -. & -- \\
\hline 726 & -- & -- & 10 & -- \\
\hline 727 & -- & -- & 25 & -- \\
\hline 728 & -- & -- & 25 & -- \\
\hline
\end{tabular}


Table 1.--Records of wells and test holes--continued.

[Dashes indicate no data. Locations shown on pls. 1A, 1B.]

\begin{tabular}{|c|c|c|c|c|c|c|c|c|c|c|}
\hline \multirow[b]{2}{*}{$\begin{array}{c}\text { Well } \\
\text { number }\end{array}$} & \multicolumn{3}{|c|}{ Location } & \multirow[b]{2}{*}{ Owner } & \multirow[b]{2}{*}{$\begin{array}{c}\text { Date } \\
\text { drilled }\end{array}$} & \multirow{2}{*}{$\begin{array}{c}\text { Altitude } \\
\text { of land } \\
\text { surface } \\
\text { (feet) }\end{array}$} & \multirow[b]{2}{*}{$\begin{array}{l}\text { Well } \\
\text { depth } \\
\text { (feet) }\end{array}$} & \multirow[b]{2}{*}{$\begin{array}{l}\text { Casing } \\
\text { depth } \\
\text { (feet) }\end{array}$} & \multirow[b]{2}{*}{$\begin{array}{c}\text { Depth } \\
\text { to } \\
\text { bedrock } \\
\text { (feet) }\end{array}$} & \multirow[b]{2}{*}{$\begin{array}{c}\text { Geologic } \\
\text { unit }\end{array}$} \\
\hline & $\begin{array}{l}\text { Lati- } \\
\text { tude } \\
\ldots .\end{array}$ & $\begin{array}{l}\text { Longi- } \\
\text { tude } \\
\ldots n\end{array}$ & $\begin{array}{l}\text { Accu- } \\
\text { racy }\end{array}$ & & & & & & & \\
\hline $\begin{array}{l}729 \\
730 \\
732 \\
734 \\
735\end{array}$ & $\begin{array}{l}424235 \\
424239 \\
424328 \\
424331 \\
424331\end{array}$ & $\begin{array}{l}0752924 \\
0752210 \\
0752947 \\
0752810 \\
0752856\end{array}$ & $\begin{array}{l}\mathrm{T} \\
\mathrm{T} \\
\mathrm{T} \\
\mathrm{T} \\
\mathrm{T}\end{array}$ & $\begin{array}{l}\text { Collins } \\
\text { Boyer } \\
\text { Griffis } \\
\text { Britton } \\
\text { Bormann }\end{array}$ & $\begin{array}{c}-\overline{-} \\
06-27-86 \\
02-01-86 \\
04-12-88 \\
04-30-87\end{array}$ & $\begin{array}{l}1170 \\
1460 \\
1190 \\
1310 \\
1160\end{array}$ & $\begin{array}{l}115 \\
159 \\
115 \\
174 \\
140\end{array}$ & $\begin{array}{l}79 \\
10 \\
50 \\
80 \\
46\end{array}$ & $\begin{array}{l}79 \\
10 \\
50 \\
80 \\
46\end{array}$ & $\begin{array}{l}\text { BDRK } \\
\text { BDRK } \\
\text { BDRK } \\
\text { BDRK } \\
\text { BDRK }\end{array}$ \\
\hline $\begin{array}{l}737 \\
738 \\
739 \\
740 \\
741\end{array}$ & $\begin{array}{l}424336 \\
424346 \\
424358 \\
424414 \\
424419\end{array}$ & $\begin{array}{l}0752715 \\
0751947 \\
0751957 \\
0752658 \\
0752704\end{array}$ & $\begin{array}{l}\mathrm{T} \\
\mathrm{T} \\
\mathrm{T} \\
\mathrm{T} \\
\mathrm{T}\end{array}$ & $\begin{array}{l}\text { Knapp } \\
\text { Kochayda } \\
\text { Kupris } \\
\text { Collins } \\
\text { Palmer }\end{array}$ & $\begin{array}{c}-- \\
06-07-89 \\
06-01-86 \\
07-22-85 \\
11-18-85\end{array}$ & $\begin{array}{l}1310 \\
1280 \\
1305 \\
1345 \\
1360\end{array}$ & $\begin{array}{r}206 \\
97 \\
98 \\
125 \\
150\end{array}$ & $\begin{array}{r}143 \\
53 \\
35 \\
50 \\
120\end{array}$ & $\begin{array}{r}143 \\
53 \\
35 \\
50 \\
120\end{array}$ & $\begin{array}{l}\text { BDRK } \\
\text { BDRK } \\
\text { BDRK } \\
\text { BDRK } \\
\text { BDRK }\end{array}$ \\
\hline $\begin{array}{l}742 \\
743 \\
745 \\
747 \\
750\end{array}$ & $\begin{array}{l}424430 \\
421818 \\
423225 \\
423203 \\
423227\end{array}$ & $\begin{array}{l}0752934 \\
0754829 \\
0752404 \\
0752357 \\
0752409\end{array}$ & $\begin{array}{l}\mathrm{T} \\
\mathrm{F} \\
\mathrm{S} \\
\mathrm{S} \\
\mathrm{S}\end{array}$ & $\begin{array}{l}\text { Winton } \\
\text { Kahn } \\
\text { NYSDOT } \\
\text { NYSDOT } \\
\text { NYSDOT }\end{array}$ & $\begin{array}{c}06-04-85 \\
-- \\
05-12-78 \\
05-10-79 \\
06-26-79\end{array}$ & $\begin{array}{l}1230 \\
1000 \\
1150 \\
1171 \\
1164\end{array}$ & $\begin{array}{r}355 \\
186 \\
76 \\
22 \\
50\end{array}$ & $\begin{array}{c}17 \\
33 \\
-- \\
-- \\
--\end{array}$ & $\begin{array}{r}17 \\
33 \\
68 \\
-- \\
41\end{array}$ & $\begin{array}{l}\text { BDRK } \\
\text { BDRK } \\
\text { BDRK } \\
\text { QRNR } \\
\text { BDRK }\end{array}$ \\
\hline $\begin{array}{l}751 \\
756 \\
761 \\
762\end{array}$ & $\begin{array}{l}423230 \\
423232 \\
423250 \\
423225\end{array}$ & $\begin{array}{l}0752428 \\
0752436 \\
0752516 \\
0752403\end{array}$ & $\begin{array}{l}S \\
S \\
S \\
S\end{array}$ & $\begin{array}{l}\text { NYSDOT } \\
\text { NYSDOT } \\
\text { NYSDOT } \\
\text { NYSDOT }\end{array}$ & $\begin{array}{l}07-10-79 \\
07-19-79 \\
12-22-77 \\
05-05-86\end{array}$ & $\begin{array}{l}1247 \\
1228 \\
1312 \\
1152\end{array}$ & $\begin{array}{l}50 \\
30 \\
42 \\
80\end{array}$ & $\begin{array}{l}-- \\
-- \\
-- \\
--\end{array}$ & $\begin{array}{r}26 \\
5 \\
38 \\
70\end{array}$ & $\begin{array}{l}\text { BDRK } \\
\text { BDRK } \\
\text { BDRK } \\
\text { BDRK }\end{array}$ \\
\hline 763 & 423234 & 0752435 & $S$ & NYSDOT & $11-28-78$ & 1220 & 19 & -- & 2 & BDRK \\
\hline $\begin{array}{l}764 \\
768 \\
770 \\
772 \\
773\end{array}$ & $\begin{array}{l}423243 \\
423251 \\
423254 \\
423259 \\
423215\end{array}$ & $\begin{array}{l}0752446 \\
0752554 \\
0752602 \\
0752616 \\
0752358\end{array}$ & $\begin{array}{l}S \\
S \\
S \\
S \\
S\end{array}$ & $\begin{array}{l}\text { NYSDOT } \\
\text { NYSDOT } \\
\text { NYSDOT } \\
\text { NYSDOT } \\
\text { NYSDOT }\end{array}$ & $\begin{array}{l}11-16-78 \\
09-03-86 \\
06-27-86 \\
08-28-86 \\
04-25-86\end{array}$ & $\begin{array}{l}1254 \\
1423 \\
1494 \\
1521 \\
1130\end{array}$ & $\begin{array}{l}32 \\
26 \\
32 \\
10 \\
42\end{array}$ & $\begin{array}{l}-- \\
-- \\
-- \\
--\end{array}$ & $\begin{array}{l}22 \\
- \\
- \\
- \\
32\end{array}$ & $\begin{array}{l}\text { BDRK } \\
\text { QRNR } \\
\text { QRNR } \\
\text { QRNR } \\
\text { BDRK }\end{array}$ \\
\hline $\begin{array}{l}774 \\
775 \\
776\end{array}$ & $\begin{array}{l}423254 \\
421539 \\
421538\end{array}$ & $\begin{array}{l}0752503 \\
0752825 \\
0752822\end{array}$ & $\begin{array}{l}S \\
F \\
F\end{array}$ & $\begin{array}{l}\text { NYSDOT } \\
\text { NYSDOT } \\
\text { NYSDOT }\end{array}$ & $\begin{array}{l}12-01-78 \\
01-21-72 \\
01-05-72\end{array}$ & $\begin{array}{r}1327 \\
978 \\
982\end{array}$ & $\begin{array}{r}39 \\
152 \\
143\end{array}$ & $\begin{array}{l}-- \\
-- \\
--\end{array}$ & $\begin{array}{r}30 \\
138 \\
132\end{array}$ & $\begin{array}{l}\text { BDRK } \\
\text { BDRK } \\
\text { BDRK }\end{array}$ \\
\hline $\begin{array}{l}777 \\
778\end{array}$ & $\begin{array}{l}421720 \\
421406\end{array}$ & $\begin{array}{l}0752821 \\
0752938\end{array}$ & $\begin{array}{l}\text { F } \\
F\end{array}$ & $\begin{array}{l}\text { NYSDOT } \\
\text { NYSDOT }\end{array}$ & $\begin{array}{l}01-04-72 \\
01-18-72\end{array}$ & $\begin{array}{l}984 \\
991\end{array}$ & $\begin{array}{l}83 \\
70\end{array}$ & -- & $\begin{array}{l}73 \\
60\end{array}$ & $\begin{array}{l}\text { BDRK } \\
\text { BDRK }\end{array}$ \\
\hline $\begin{array}{l}779 \\
780 \\
781 \\
782 \\
783\end{array}$ & $\begin{array}{l}423954 \\
421904 \\
421903 \\
423623 \\
423607\end{array}$ & $\begin{array}{l}0755234 \\
0753927 \\
0753900 \\
0753920 \\
0753820\end{array}$ & $\begin{array}{l}F \\
F \\
F \\
F \\
F\end{array}$ & $\begin{array}{l}\text { Solvent Savers } \\
\text { NYSDOT } \\
\text { NYSDOT } \\
\text { NYSDOT } \\
\text { NYSDOT }\end{array}$ & $\begin{array}{l}09-23-84 \\
08-28-63 \\
09-06-63 \\
08-30-63 \\
01-06-67\end{array}$ & $\begin{array}{l}1167 \\
1261 \\
1470 \\
1403 \\
1332\end{array}$ & $\begin{array}{r}102 \\
16 \\
24 \\
50 \\
20\end{array}$ & $\begin{array}{l}-- \\
- \\
- \\
- \\
--\end{array}$ & $\begin{array}{l}-- \\
-- \\
-- \\
10\end{array}$ & $\begin{array}{l}\text { QRNR } \\
\text { BDRK } \\
\text { QRNR } \\
\text { QRNR } \\
\text { BDRK }\end{array}$ \\
\hline $\begin{array}{l}784 \\
785 \\
786 \\
787 \\
788\end{array}$ & $\begin{array}{l}423756 \\
422437 \\
422438 \\
422435 \\
422436\end{array}$ & $\begin{array}{l}0754736 \\
0753231 \\
0753117 \\
0753353 \\
0753009\end{array}$ & $\begin{array}{l}S \\
F \\
F \\
F \\
F\end{array}$ & $\begin{array}{l}\text { NYSDOT } \\
\text { NYSDOT } \\
\text { NYSDOT } \\
\text { NYSDOT } \\
\text { NYSDOT }\end{array}$ & $\begin{array}{l}06-26-59 \\
04-14-82 \\
01-30-76 \\
06-09-82 \\
02-25-71\end{array}$ & $\begin{array}{l}1205 \\
1350 \\
1537 \\
1490 \\
1588\end{array}$ & $\begin{array}{l}69 \\
16 \\
22 \\
16 \\
22\end{array}$ & $\begin{array}{l}-- \\
- \\
- \\
- \\
--\end{array}$ & $\begin{array}{l}-- \\
-- \\
-- \\
- \\
2\end{array}$ & $\begin{array}{l}\text { QRNR } \\
\text { QRNR } \\
\text { QRNR } \\
\text { QRNR } \\
\text { BDRK }\end{array}$ \\
\hline $\begin{array}{l}789 \\
790 \\
791 \\
792 \\
793\end{array}$ & $\begin{array}{l}422439 \\
422440 \\
422540 \\
422517 \\
421847\end{array}$ & $\begin{array}{l}0753032 \\
0753057 \\
0753437 \\
.0753407 \\
0753557\end{array}$ & $\begin{array}{l}F \\
F \\
F \\
F \\
F\end{array}$ & $\begin{array}{l}\text { NYSDOT } \\
\text { NYSDOT } \\
\text { NYSDOT } \\
\text { NYSDOT } \\
\text { NYSDOT }\end{array}$ & $\begin{array}{l}03-10-71 \\
05-18-71 \\
03-10-82 \\
09-20-78 \\
04-14-86\end{array}$ & $\begin{array}{l}1628 \\
1598 \\
1418 \\
1569 \\
1436\end{array}$ & $\begin{array}{l}20 \\
42 \\
16 \\
19 \\
15\end{array}$ & $\begin{array}{l}-- \\
-- \\
-- \\
--\end{array}$ & $\begin{array}{c}12 \\
32 \\
-- \\
17 \\
--\end{array}$ & $\begin{array}{l}\text { BDRK } \\
\text { BDRK } \\
\text { QRNR } \\
\text { BDRK } \\
\text { QRNR }\end{array}$ \\
\hline $\begin{array}{l}794 \\
795 \\
796 \\
797 \\
798\end{array}$ & $\begin{array}{l}421849 \\
421849 \\
421936 \\
421938 \\
422433\end{array}$ & $\begin{array}{l}0753548 \\
0753630 \\
0754546 \\
0754602 \\
0752945\end{array}$ & $\begin{array}{l}F \\
F \\
F \\
F \\
F\end{array}$ & $\begin{array}{l}\text { NYSDOT } \\
\text { NYSDOT } \\
\text { NYSDOT } \\
\text { NYSDOT } \\
\text { NYSDOT }\end{array}$ & $\begin{array}{l}04-18-86 \\
09-29-71 \\
08-15-63 \\
08-07-63 \\
01-22-71\end{array}$ & $\begin{array}{r}1408 \\
1385 \\
1135 \\
916 \\
1579\end{array}$ & $\begin{array}{l}40 \\
52 \\
40 \\
17 \\
20\end{array}$ & $\begin{array}{l}-- \\
- \\
-- \\
-- \\
-\end{array}$ & $\begin{array}{c}36 \\
-- \\
-- \\
- \\
10\end{array}$ & $\begin{array}{l}\text { BDRK } \\
\text { QRNR } \\
\text { QRNR } \\
\text { QRNR } \\
\text { BDRK }\end{array}$ \\
\hline
\end{tabular}


Table 1.--Records of wells and test holes--continued.

[Dashes indicate no data. Locations shown on pls. 1A, 1B.]

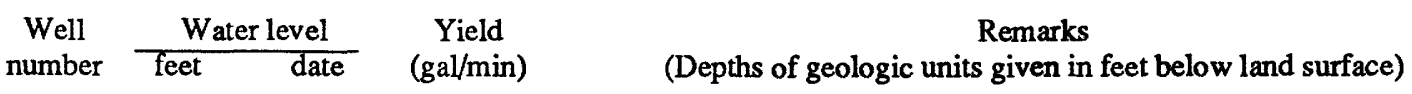

\begin{tabular}{|c|c|c|c|c|}
\hline $\begin{array}{l}729 \\
730 \\
732 \\
734 \\
735\end{array}$ & $\begin{array}{l}-- \\
-- \\
-- \\
-- \\
--\end{array}$ & $\begin{array}{l}-- \\
- \\
- \\
- \\
-\end{array}$ & $\begin{array}{r}10 \\
8 \\
-- \\
6 \\
20\end{array}$ & $\begin{array}{l}-- \\
-- \\
-- \\
-- \\
--\end{array}$ \\
\hline $\begin{array}{l}737 \\
738 \\
739 \\
740 \\
741\end{array}$ & $\begin{array}{l}-- \\
-- \\
-- \\
--\end{array}$ & $\begin{array}{l}-- \\
-- \\
- \\
-- \\
--\end{array}$ & $\begin{array}{r}12 \\
5 \\
-- \\
-- \\
9\end{array}$ & $\begin{array}{l}-- \\
-- \\
-- \\
-- \\
--\end{array}$ \\
\hline $\begin{array}{l}742 \\
743 \\
745 \\
747 \\
750\end{array}$ & $\begin{array}{l}-- \\
- \\
6 \\
8 \\
9\end{array}$ & $\begin{array}{c}-- \\
-- \\
05-12-78 \\
05-10-79 \\
06-26-79\end{array}$ & $\begin{array}{r}2 \\
10 \\
-- \\
-- \\
--\end{array}$ & $\begin{array}{l}- \\
\overline{-} \\
\overline{0-5} \text { sandy silt, gravelly; 5-22 sandy gravel. } \\
0-20 \text { gravel; } 20-26 \text { sand; } 26-31 \text { gravel; } 31-36 \text { sand; } 36-41 \text { gravel; } 41 \mathrm{SH} .\end{array}$ \\
\hline $\begin{array}{l}751 \\
756 \\
761 \\
762\end{array}$ & $\begin{array}{l}-- \\
4 \\
4 \\
6\end{array}$ & $\begin{array}{l}-\overline{07-19-79} \\
12-22-77 \\
05-06-86\end{array}$ & $\begin{array}{l}-- \\
-- \\
--\end{array}$ & $\begin{array}{l}0-26 \text { sandy gravel; } 26 \text { BR. Hole caved in at } 26 \mathrm{ft} \text {. } \\
0-5 \text { sandy silt; } 5-30 \text { gray SH. } \\
0-10 \text { gravel; } 10-20 \text { clayey silt; } 20-38 \text { coarse silt; } 38-42 \text { boulder \& SS. } \\
0-26 \text { gravel; } 26-42 \text { sandy silt; } 42-47 \text { clayey silt; } 47-63 \text { sandy silt; } 63-70 \text { silty } \\
\text { gravel. } \\
0-2 \text { sandy gravel: } 2-9 \text { rotten } \mathrm{SH}: 9-19 \mathrm{SH} \text {. }\end{array}$ \\
\hline $\begin{array}{l}763 \\
764 \\
768 \\
770 \\
772 \\
773\end{array}$ & $\begin{array}{r}1 \\
6 \\
8 \\
20 \\
- \\
5\end{array}$ & $\begin{array}{l}11-28-78 \\
11-16-78 \\
09-03-86 \\
06-27-86 \\
08-28-86 \\
04-25-86\end{array}$ & $\begin{array}{l}-- \\
-- \\
-- \\
- \\
-\end{array}$ & $\begin{array}{l}0-2 \text { sandy gravel; } 2-9 \text { rotten } \mathrm{SH} ; 9-19 \text { SH. } \\
0-6 \text { sandy gravel; } 6-22 \text { clayey silt; } 22 \text { broken } \text { SS. } \\
0-2 \text { sandy silt; } 2-14 \text { gravelly silt; } 14-26 \text { silty gravel. } \\
0-2 \text { sand; } 2-14 \text { silt; } 14-21 \text { gravelly sand; } 21-30 \text { sandy silt. } \\
0-10 \text { gravelly silt w/sand. } \\
0-2 \text { sand; } 2-4 \text { silt; } 4-6 \text { gravel; } 6-20 \text { coarse silt; } 20-32 \text { gravel; } 32-42 \text { SH. }\end{array}$ \\
\hline $\begin{array}{l}774 \\
775 \\
776\end{array}$ & $\begin{array}{l}9 \\
7 \\
7\end{array}$ & $\begin{array}{l}12-01-78 \\
01-21-72 \\
01-05-72\end{array}$ & $\begin{array}{l}-- \\
-- \\
-\end{array}$ & $\begin{array}{l}\text { 0-10 sandy gravel; } 10-26 \text { sandy silt; } 26-30 \text { clayey silt; } 30-39 \text { SS \& SH. } \\
0-10 \text { gravel; } 10-136 \text { silt; } 136-145 \text { fine sand; } 145-152 \text { SS \& SH. } \\
\text { 0-10 gravel; } 10-15 \text { sand; } 15-119 \text { silt; } 119-124 \text { sand; } 124-132 \text { silt; } 132-143 \text { SH \& } \\
\text { LS. }\end{array}$ \\
\hline $\begin{array}{l}777 \\
778\end{array}$ & $\begin{array}{l}17 \\
24\end{array}$ & $\begin{array}{l}01-04-72 \\
01-18-72\end{array}$ & $\begin{array}{l}-- \\
--\end{array}$ & $\begin{array}{l}0-10 \text { gravel; } 10-15 \text { sand; } 15-65 \text { silt; } 65-73 \text { sand; } 73-83 \mathrm{SS} \& \mathrm{SH} \text {. } \\
0-10 \text { gravel; } 10-40 \text { fine sand; } 40-45 \text { silt; } 45-50 \text { fine sand; } 50-60 \text { gravel; } 60-70 \mathrm{SS} \\
\text { \& SH. }\end{array}$ \\
\hline $\begin{array}{l}779 \\
780 \\
781 \\
782 \\
783\end{array}$ & $\begin{array}{r}9 \\
3 \\
8 \\
15 \\
3\end{array}$ & $\begin{array}{l}09-23-84 \\
08-28-63 \\
09-06-63 \\
08-30-63 \\
01-06-67\end{array}$ & $\begin{array}{l}-- \\
-- \\
- \\
--\end{array}$ & $\begin{array}{l}0-3 \text { silt; } 3-58 \text { sand \& gravel; } 58-102 \text { silt, fine to coarse sand and gravel. } \\
0-4 \text { sand and stone; } 4-6 \text { rotten } \mathrm{SH} ; 6-16 \mathrm{SS} \text { \& } \mathrm{SH} \text {. } \\
0-19 \text { sand, clay, \& stone; } 19-24 \text { silt w/some stones. } \\
0-50 \text { silt w/sand, clay, and stone. } \\
0-10 \text { gravel, some sand, trace silt; } 10-20 \mathrm{SH} \text {. }\end{array}$ \\
\hline $\begin{array}{l}784 \\
785 \\
786 \\
787 \\
788\end{array}$ & $\begin{array}{l}8 \\
4 \\
2 \\
8 \\
2\end{array}$ & $\begin{array}{l}06-26-59 \\
04-14-82 \\
01-30-76 \\
06-09-82 \\
02-25-71\end{array}$ & $\begin{array}{l}-- \\
-- \\
- \\
- \\
-\end{array}$ & $\begin{array}{l}0-18 \text { sand, gravel, \& silt; } 18-24 \text { coarse sand; } 24-69 \text { silt \& sand. } \\
0-6 \text { sandy silt; } 6-10 \text { clayey silt; } 10-16 \text { sandy gravel. } \\
0-22 \text { gravelly silt. } \\
0-5 \text { sandy silt; } 5-10 \text { sandy gravel; } 10-16 \text { silty clay. } \\
0-2 \text { gravel; } 2-22 \text { rotten SH. }\end{array}$ \\
\hline $\begin{array}{l}789 \\
790 \\
791 \\
792 \\
793\end{array}$ & $\begin{array}{r}9 \\
8 \\
3 \\
11 \\
8\end{array}$ & $\begin{array}{l}03-10-71 \\
05-18-71 \\
03-10-82 \\
09-20-78 \\
04-14-86\end{array}$ & $\begin{array}{l}-- \\
-- \\
- \\
- \\
-\end{array}$ & $\begin{array}{l}0-5 \text { sand \& gravel; } 5-12 \text { gravel \& silt; } 12-20 \text { rotten } \mathrm{SH} \text {. } \\
0-21 \text { sand \& gravel; } 21-31 \text { silt; } 31-32 \text { sand \& gravel; } 32-42 \mathrm{SH} . \\
0-15 \text { gravelly silt; } 15-16 \text { sandy gravel. } \\
0-5 \text { gravelly silt; } 5-15 \text { sandy silt; } 15-17 \text { gravelly silt; } 17-19 \text { SH \& SS. } \\
0-10 \text { gravelly silt; } 10-15 \text { gravelly silt. }\end{array}$ \\
\hline $\begin{array}{l}794 \\
795 \\
796 \\
797 \\
798\end{array}$ & $\begin{array}{l}24 \\
20 \\
19 \\
10 \\
10\end{array}$ & $\begin{array}{l}04-18-86 \\
09-29-71 \\
08-15-63 \\
08-07-63 \\
01-22-71\end{array}$ & $\begin{array}{l}-- \\
-- \\
-- \\
- \\
-\end{array}$ & $\begin{array}{l}0-20 \text { gravelly silt; } 20-36 \text { silt \& gravel; } 36-40 \mathrm{SH} \text { w/shells. } \\
0-51 \text { sand \& gravel; } 51-52 \text { silt \& sand, some gravel. } \\
0-9 \text { sand, clay, and stone; } 9-32 \text { sand \& gravel; } 32-40 \text { clay, sand, \& some stone. } \\
0-8 \text { sand; } 8-13 \text { silt, sand \& organic matter; } 13-17 \text { sand, silt, \& organic matter. } \\
0-5 \text { silt, some sand; 5-10 sand \& gravel; } 10-20 \mathrm{SH} \text {. }\end{array}$ \\
\hline
\end{tabular}


Table 1.--Records of wells and test holes--continued.

[Dashes indicate no data. Locations shown on pls. 1A, 1B.]

\begin{tabular}{|c|c|c|c|c|c|c|c|c|c|c|}
\hline \multirow[b]{2}{*}{$\begin{array}{c}\text { Well } \\
\text { number }\end{array}$} & \multicolumn{3}{|c|}{ Location } & \multirow[b]{2}{*}{ Owner } & \multirow[b]{2}{*}{$\begin{array}{c}\text { Date } \\
\text { drilled }\end{array}$} & \multirow{2}{*}{$\begin{array}{c}\text { Altitude } \\
\text { of land } \\
\text { surface } \\
\text { (feet) }\end{array}$} & \multirow[b]{2}{*}{$\begin{array}{l}\text { Well } \\
\text { depth } \\
\text { (feet) }\end{array}$} & \multirow[b]{2}{*}{$\begin{array}{l}\text { Casing } \\
\text { depth } \\
\text { (feet) }\end{array}$} & \multirow{2}{*}{$\begin{array}{l}\text { Depth } \\
\text { to } \\
\text { bedrock } \\
\text { (feet) }\end{array}$} & \multirow[b]{2}{*}{$\begin{array}{c}\text { Geologic } \\
\text { unit }\end{array}$} \\
\hline & $\begin{array}{l}\text { Lati- } \\
\text { tude } \\
\cdots n\end{array}$ & $\begin{array}{c}\text { Longi- } \\
\text { tude } \\
\ldots\end{array}$ & $\begin{array}{l}\text { Accu- } \\
\text { racy }\end{array}$ & & & & & & & \\
\hline $\begin{array}{l}799 \\
800 \\
801 \\
802 \\
803\end{array}$ & $\begin{array}{l}422412 \\
422318 \\
422416 \\
422417 \\
422409\end{array}$ & $\begin{array}{l}0752911 \\
0752417 \\
0752843 \\
0752755 \\
0752741\end{array}$ & $\begin{array}{l}\text { F } \\
F \\
F \\
F \\
F\end{array}$ & $\begin{array}{l}\text { NYSDOT } \\
\text { NYSDOT } \\
\text { NYSDOT } \\
\text { NYSDOT } \\
\text { NYSDOT }\end{array}$ & $\begin{array}{c}05-03-71 \\
-- \\
-- \\
11-30-70 \\
04-20-71\end{array}$ & $\begin{array}{l}1500 \\
1022 \\
1455 \\
1420 \\
1407\end{array}$ & $\begin{array}{l}40 \\
57 \\
28 \\
20 \\
25\end{array}$ & $\begin{array}{l}-- \\
-- \\
-- \\
--\end{array}$ & $\begin{array}{r}-- \\
-- \\
15 \\
6 \\
13\end{array}$ & $\begin{array}{l}\text { QRNR } \\
\text { QRNR } \\
\text { BDRK } \\
\text { BDRK } \\
\text { BDRK }\end{array}$ \\
\hline $\begin{array}{l}804 \\
805 \\
806 \\
808 \\
809\end{array}$ & $\begin{array}{l}422354 \\
422342 \\
422329 \\
424227 \\
422629\end{array}$ & $\begin{array}{l}0752733 \\
0752706 \\
0752628 \\
0753727 \\
0753537\end{array}$ & $\begin{array}{l}F \\
F \\
F \\
F \\
F\end{array}$ & $\begin{array}{l}\text { NYSDOT } \\
\text { NYSDOT } \\
\text { NYSDOT } \\
\text { NYSDOT } \\
\text { NYSDOT }\end{array}$ & $\begin{array}{l}05-27-71 \\
12-15-70 \\
04-12-71 \\
02-03-36 \\
07-30-38\end{array}$ & $\begin{array}{r}1396 \\
1402 \\
1373 \\
1404 \\
965\end{array}$ & $\begin{array}{l}18 \\
52 \\
44 \\
32 \\
25\end{array}$ & $\begin{array}{l}-- \\
-- \\
-- \\
--\end{array}$ & $\begin{array}{c}6 \\
-- \\
-- \\
22 \\
--\end{array}$ & $\begin{array}{l}\text { BDRK } \\
\text { QRNR } \\
\text { QRNR } \\
\text { BDRK } \\
\text { QRNR }\end{array}$ \\
\hline $\begin{array}{l}810 \\
811 \\
812 \\
813 \\
814\end{array}$ & $\begin{array}{l}424127 \\
424305 \\
422002 \\
421951 \\
422818\end{array}$ & $\begin{array}{l}0753014 \\
0753225 \\
0754747 \\
0754744 \\
0753517\end{array}$ & $\begin{array}{l}F \\
F \\
F \\
F \\
F\end{array}$ & $\begin{array}{l}\text { NYSDOT } \\
\text { NYSDOT } \\
\text { NYSDOT } \\
\text { NYSDOT } \\
\text { NYSDOT }\end{array}$ & $\begin{array}{l}02-04-55 \\
02-25-55 \\
08-12-47 \\
08-12-47 \\
10-11-85\end{array}$ & $\begin{array}{r}1053 \\
1064 \\
947 \\
1025 \\
1086\end{array}$ & $\begin{array}{r}102 \\
121 \\
115 \\
105 \\
38\end{array}$ & $\begin{array}{l}-- \\
-- \\
-- \\
--\end{array}$ & $\begin{array}{r}-- \\
121 \\
-- \\
23 \\
25\end{array}$ & $\begin{array}{l}\text { QRNR } \\
\text { BDRK } \\
\text { QRNR } \\
\text { BDRK } \\
\text { BDRK }\end{array}$ \\
\hline $\begin{array}{l}815 \\
816 \\
817 \\
818 \\
819\end{array}$ & $\begin{array}{l}422816 \\
424423 \\
423053 \\
424318 \\
422052\end{array}$ & $\begin{array}{l}0753518 \\
0753207 \\
0753019 \\
0754015 \\
0754816\end{array}$ & $\begin{array}{l}\mathbf{F} \\
\mathbf{F} \\
\mathbf{F} \\
\mathbf{F} \\
\mathbf{F}\end{array}$ & $\begin{array}{l}\text { NYSDOT } \\
\text { NYSDOT } \\
\text { NYSDOT } \\
\text { NYSDOT } \\
\text { NYSDOT }\end{array}$ & $\begin{array}{l}10-17-85 \\
03-24-86 \\
07-16-86 \\
04-07-86 \\
08-30-88\end{array}$ & $\begin{array}{r}1084 \\
1080 \\
1059 \\
1504 \\
938\end{array}$ & $\begin{array}{r}34 \\
131 \\
31 \\
42 \\
74\end{array}$ & $\begin{array}{l}-- \\
-- \\
-- \\
--\end{array}$ & $\begin{array}{r}20 \\
-- \\
21 \\
-- \\
64\end{array}$ & $\begin{array}{l}\text { BDRK } \\
\text { QRNR } \\
\text { BDRK } \\
\text { QRNR } \\
\text { BDRK }\end{array}$ \\
\hline $\begin{array}{l}820 \\
821 \\
822 \\
824\end{array}$ & $\begin{array}{l}423734 \\
424104 \\
424049 \\
423849\end{array}$ & $\begin{array}{l}0751940 \\
0751916 \\
0752747 \\
0753157\end{array}$ & $\begin{array}{l}\text { F } \\
\text { F } \\
\text { F } \\
\text { S }\end{array}$ & $\begin{array}{l}\text { NYSDOT } \\
\text { NYSDOT } \\
\text { NYSDOT } \\
\text { USGS }\end{array}$ & $\begin{array}{l}01-30-89 \\
08-13-85 \\
03-22-84 \\
11-01-80\end{array}$ & $\begin{array}{l}1099 \\
1110 \\
1256 \\
1066\end{array}$ & $\begin{array}{r}100 \\
94 \\
17 \\
125\end{array}$ & $\begin{array}{r}-- \\
-- \\
-- \\
121\end{array}$ & $\begin{array}{r}-- \\
-- \\
155\end{array}$ & $\begin{array}{l}\text { QRNR } \\
\text { QRNR } \\
\text { BDRK } \\
\text { BDRK }\end{array}$ \\
\hline 825 & 421342 & 0753137 & - & Afton Dist. Sys. & - & - & -- & - & -- & - \\
\hline $\begin{array}{l}826 \\
827 \\
828 \\
829 \\
830\end{array}$ & $\begin{array}{l}421946 \\
422634 \\
423725 \\
423125 \\
423135\end{array}$ & $\begin{array}{l}0754615 \\
0753549 \\
0751957 \\
0753053 \\
0753054\end{array}$ & $\begin{array}{l}- \\
- \\
\bar{F} \\
F\end{array}$ & $\begin{array}{l}\text { Greene Dist. Sys. } \\
\text { Oxford Dist. Sys. } \\
\text { New Berlin Dist. Sys. } \\
\text { City of Norwich } \\
--\end{array}$ & $\begin{array}{l}-- \\
-- \\
-- \\
--\end{array}$ & $\begin{array}{r}-- \\
-- \\
994 \\
997\end{array}$ & $\begin{array}{r}-- \\
-- \\
67 \\
102\end{array}$ & $\begin{array}{l}-- \\
-- \\
-- \\
-- \\
--\end{array}$ & $\begin{array}{l}-- \\
-- \\
-- \\
-- \\
--\end{array}$ & $\begin{array}{l}-- \\
-- \\
-- \\
\text { QRNR } \\
\text { QRNR }\end{array}$ \\
\hline $\begin{array}{l}831 \\
832 \\
833 \\
834 \\
835\end{array}$ & $\begin{array}{l}423121 \\
423130 \\
422438 \\
422300 \\
422357\end{array}$ & $\begin{array}{l}0753055 \\
0753055 \\
0754822 \\
0754613 \\
0754612\end{array}$ & $\begin{array}{l}\text { F } \\
\text { F } \\
\text { F } \\
\text { F } \\
\text { F }\end{array}$ & $\begin{array}{l}-- \\
\text { Urda } \\
\text { Decker } \\
\text { Decker }\end{array}$ & $\begin{array}{c}-- \\
-- \\
01-01-64 \\
09-15-64 \\
03-05-64\end{array}$ & $\begin{array}{r}990 \\
- \\
1058 \\
1488 \\
1370\end{array}$ & $\begin{array}{r}57 \\
72 \\
335 \\
3650 \\
3265\end{array}$ & $\begin{array}{r}-- \\
-- \\
19 \\
555 \\
950\end{array}$ & $\begin{array}{c}-- \\
-- \\
19 \\
15 \\
44\end{array}$ & $\begin{array}{l}\text { QRNR } \\
\text { QRNR } \\
\text { BDRK } \\
\text { BDRK } \\
\text { BDRK }\end{array}$ \\
\hline $\begin{array}{l}836 \\
837 \\
838 \\
839 \\
840\end{array}$ & $\begin{array}{l}422338 \\
422327 \\
422320 \\
422305 \\
422314\end{array}$ & $\begin{array}{l}0754642 \\
0754618 \\
0754647 \\
0754759 \\
0754627\end{array}$ & $\begin{array}{l}\text { F } \\
\text { F } \\
\text { F } \\
\text { F } \\
\text { F }\end{array}$ & $\begin{array}{l}\text { Decker } \\
\text { Decker } \\
\text { Bottle } \\
\text { Flanigan } \\
\text { Bottle }\end{array}$ & $\begin{array}{c}10-28-64 \\
1964 \\
06-05-65 \\
07-23-65 \\
09-03-65\end{array}$ & $\begin{array}{l}1495 \\
1534 \\
1485 \\
1140 \\
1485\end{array}$ & $\begin{array}{l}1940 \\
2038 \\
1985 \\
2462 \\
2100\end{array}$ & $\begin{array}{r}1940 \\
2000 \\
1926 \\
600 \\
1943\end{array}$ & $\begin{array}{r}12 \\
6 \\
10 \\
40 \\
13\end{array}$ & $\begin{array}{l}\text { BDRK } \\
\text { BDRK } \\
\text { BDRK } \\
\text { BDRK } \\
\text { BDRK }\end{array}$ \\
\hline $\begin{array}{l}841 \\
842 \\
843 \\
844 \\
845\end{array}$ & $\begin{array}{l}422332 \\
422703 \\
422219 \\
421903 \\
422051\end{array}$ & $\begin{array}{l}0754556 \\
0752908 \\
0755018 \\
0754019 \\
0753520\end{array}$ & $\begin{array}{l}F \\
F \\
F \\
F \\
F\end{array}$ & $\begin{array}{l}\text { Bell } \\
\text { Wahlberg } \\
\text { Clark } \\
\text { Small } \\
\text { Hulbert }\end{array}$ & $\begin{array}{l}10-21-65 \\
05-06-74 \\
12-27-65 \\
06-06-74 \\
05-20-74\end{array}$ & $\begin{array}{l}1325 \\
1717 \\
1268 \\
1360 \\
1694\end{array}$ & $\begin{array}{r}2100 \\
5181 \\
380 \\
6620 \\
6427\end{array}$ & $\begin{array}{l}605 \\
993 \\
600 \\
910 \\
968\end{array}$ & $\begin{array}{r}110 \\
70 \\
130 \\
20 \\
40\end{array}$ & $\begin{array}{l}\text { BDRK } \\
\text { BDRK } \\
\text { BDRK } \\
\text { BDRK } \\
\text { BDRK }\end{array}$ \\
\hline $\begin{array}{l}846 \\
847 \\
848 \\
849 \\
850\end{array}$ & $\begin{array}{l}422720 \\
422717 \\
422717 \\
422712 \\
423044\end{array}$ & $\begin{array}{l}0753231 \\
0753241 \\
0753310 \\
0753301 \\
0755105\end{array}$ & $\begin{array}{l}\mathbf{F} \\
\mathbf{F} \\
\mathbf{F} \\
\mathbf{F} \\
\mathbf{F}\end{array}$ & $\begin{array}{l}\text { Oxford C\&D Landfill } \\
\text { Oxford C\&D Landfill } \\
\text { Oxford C\&D Landfill } \\
\text { Oxford C\&D Landfill } \\
\text { German Landfill }\end{array}$ & $\begin{array}{l}04-24-89 \\
04-24-89 \\
05-03-89 \\
05-12-89 \\
10-25-89\end{array}$ & $\begin{array}{l}1200 \\
1193 \\
1142 \\
1228 \\
1608\end{array}$ & $\begin{array}{r}9 \\
15 \\
65 \\
22 \\
30\end{array}$ & $\begin{array}{l}-- \\
-- \\
-- \\
--\end{array}$ & $\begin{array}{r}9 \\
15 \\
-- \\
-- \\
20\end{array}$ & $\begin{array}{l}\text { BDRK } \\
\text { BDRK } \\
\text { QRNR } \\
\text { QRNR } \\
\text { BDRK }\end{array}$ \\
\hline
\end{tabular}


Table 1.--Records of wells and test holes--continued.

[Dashes indicate no data. Locations shown on pls. 1A, 1B.]

\begin{tabular}{|c|c|c|c|c|}
\hline \multirow{2}{*}{$\begin{array}{l}\text { Well } \\
\text { number }\end{array}$} & \multicolumn{2}{|c|}{ Water level } & \multirow{2}{*}{$\begin{array}{l}\text { Yield } \\
\text { (gal/min) }\end{array}$} & \multirow{2}{*}{$\begin{array}{l}\text { Remarks } \\
\text { (Depths of geologic units given in feet below land surface) }\end{array}$} \\
\hline & feet & date & & \\
\hline $\begin{array}{l}799 \\
800 \\
801 \\
802 \\
803\end{array}$ & $\begin{array}{l}12 \\
-. \\
7 \\
6 \\
4\end{array}$ & $\begin{array}{l}05-03-71 \\
-- \\
05-03-71 \\
11-30-70 \\
04-20-71\end{array}$ & $\begin{array}{l}-- \\
-- \\
-- \\
--\end{array}$ & $\begin{array}{l}\text { 0-40 mostly sand mixed w/silt \& gravel. } \\
0-57 \text { sand \& gravel (10 percent average silt). } \\
0-15 \text { sand \& gravel, some silt; } 15-18 \text { SH; } 18-28 \text { SS. } \\
0-6 \text { sand \& gravel, some silt; } 6-20 \text { rotten red SH. } \\
0-13 \text { sand \& gravel, some silt; } 13-25 \text { red SH. }\end{array}$ \\
\hline $\begin{array}{l}804 \\
805 \\
806 \\
808 \\
809\end{array}$ & $\begin{array}{r}3 \\
13 \\
12 \\
-- \\
--\end{array}$ & $\begin{array}{l}05-27-71 \\
12-15-70 \\
04-12-71 \\
02-03-36 \\
07-30-38\end{array}$ & $\begin{array}{l}-- \\
-- \\
-- \\
--\end{array}$ & $\begin{array}{l}\text { 0-6 silt \& SS; } 6-18 \text { red } S H . \\
0-15 \text { silt \& gravel; } 15-52 \text { sand \& gravel. } \\
0-37 \text { sand \& gravel; } 37-42 \text { silt; } 42-44 \text { sand \& gravel. } \\
0-9 \text { silt; } 9-22 \text { HP; } 22-32 \text { gray rock. } \\
0-12 \text { clayey silt \& sand; } 12-25 \text { sandy gravel. }\end{array}$ \\
\hline $\begin{array}{l}810 \\
811 \\
812 \\
813 \\
814\end{array}$ & $\begin{array}{c}-- \\
\overline{3} \\
-- \\
\overline{12}\end{array}$ & $\begin{array}{l}02-\overline{-} 5-55 \\
-- \\
10-\overline{11-85}\end{array}$ & $\begin{array}{l}-- \\
-- \\
-- \\
-- \\
-\end{array}$ & $\begin{array}{l}0-6 \text { sandy gravel; } 6-96 \text { silt; } 96-102 \text { sand \& silt. } \\
0-8 \text { clay; } 8-26 \text { gravel; } 26-121 \text { silt; } 121 \text { rock? } \\
0 \text { to approximately } 6 \text { sand \& gravel; } 6-115 \text { silt. } \\
0-23 \text { gravel, some sand; } 23-105 \text { SS \& } \text { SH in alternating bands. } \\
0-10 \text { silty gravel; } 10-15 \text { gravelly silt; } 15-20 \text { silty gravel; } 20-25 \text { gravelly silt; } \\
25-38 \text { SH. }\end{array}$ \\
\hline $\begin{array}{l}815 \\
816 \\
817 \\
818 \\
819\end{array}$ & $\begin{array}{r}9 \\
3 \\
17 \\
3 \\
2\end{array}$ & $\begin{array}{l}10-17-85 \\
03-24-86 \\
07-16-86 \\
04-07-86 \\
08-30-88\end{array}$ & $\begin{array}{l}-- \\
-- \\
-- \\
--\end{array}$ & $\begin{array}{l}0-6 \text { gravelly silt; } 6-14 \text { silty gravel; } 14-20 \text { gravelly silt; } 20-34 \text { SH. } \\
0-40 \text { mostly silt; } 40-55 \text { silty sand; } 55-131 \text { silt. } \\
0-5 \text { silty gravel; } 5-15 \text { silt; } 15-21 \text { silty gravel; } 21-31 \text { SH. } \\
0-2 \text { silt; } 2-30 \text { gravelly silt; } 30-42 \text { clayey silt. } \\
0-20 \text { silty gravel; } 20-52 \text { silt; } 52-64 \text { gravelly silt; } 64-74 \text { SS. }\end{array}$ \\
\hline $\begin{array}{l}820 \\
821 \\
822 \\
824\end{array}$ & $\begin{array}{r}12 \\
8 \\
13 \\
11\end{array}$ & $\begin{array}{l}01-30-89 \\
08-13-85 \\
03-22-84 \\
01-27-81\end{array}$ & $\begin{array}{c}-- \\
-- \\
30\end{array}$ & $\begin{array}{l}0-10 \text { ash; } 10-25 \text { silty gravel; } 25-100 \text { silt. } \\
0-8 \text { silt \& gravel; } 8-14 \text { silt; } 14-21 \text { sand; } 21-57 \text { silt; } 57-68 \text { sand; } 68-94 \text { coarse silt. } \\
0-5 \text { sandy silt; } 5-7 \text { gravelly silt; } 7-17 \text { SH. } \\
\text { 0-67 sand \& gravel; } 67-80 \text { sand; } 80-150 \text { sand \& gravel; } 150-155 \text { silt; } 155-164 \\
\text { STS. }\end{array}$ \\
\hline 825 & - & -- & -- & -- \\
\hline $\begin{array}{l}826 \\
827 \\
828 \\
829 \\
830\end{array}$ & $\begin{array}{l}-- \\
-- \\
-- \\
-\end{array}$ & $\begin{array}{l}-- \\
-- \\
-- \\
-- \\
--\end{array}$ & $\begin{array}{l}-- \\
-- \\
-- \\
-- \\
--\end{array}$ & $\begin{array}{l}-- \\
-- \\
-- \\
0-5 \text { clayey silt, fine sand; } 5-21 \text { silty sand \& gravel; } 21-67 \text { clay w/silt lenses. } \\
0-8 \text { clay; } 8-14 \text { sand \& gravel; } 14-28 \text { sand w/silt \& gravel; } 28-40 \text { silty; } 40-102 \\
\text { clay w/silt. }\end{array}$ \\
\hline $\begin{array}{l}831 \\
832 \\
833 \\
834 \\
835\end{array}$ & $\begin{array}{c}-- \\
40 \\
-- \\
--\end{array}$ & $\begin{array}{c}-- \\
-- \\
01-01-64 \\
-- \\
--\end{array}$ & $\begin{array}{l}-- \\
-- \\
-- \\
-- \\
--\end{array}$ & $\begin{array}{l}0-5 \text { silt; 5-24 sand, gravel, silt, \& clay layers; } 24-57 \text { silt w/clay layers. } \\
0-3 \text { fill; } 3-7 \text { clayey silt w/gravel; } 7-13 \text { sandy gravel, trace silt; } 13-72 \text { clayey silt. } \\
\text { Salt water, pumped two days, no change. Nearby well } 110 \mathrm{ft} \text { deep, no salt. } \\
\text {-- } \\
\text {-- }\end{array}$ \\
\hline $\begin{array}{l}836 \\
837 \\
838 \\
839 \\
840\end{array}$ & $\begin{array}{c}-- \\
50 \\
--\end{array}$ & $\begin{array}{l}-- \\
-- \\
06-05-65 \\
-- \\
--\end{array}$ & $\begin{array}{l}-- \\
-- \\
- \\
- \\
-\end{array}$ & $\begin{array}{l}-- \\
-- \\
-- \\
--\end{array}$ \\
\hline $\begin{array}{l}841 \\
842 \\
843 \\
844 \\
845\end{array}$ & $\begin{array}{r}65 \\
-- \\
120 \\
-- \\
--\end{array}$ & $\begin{array}{c}10-21-65 \\
-- \\
12-27-65 \\
-- \\
--\end{array}$ & $\begin{array}{l}-- \\
-- \\
-- \\
-- \\
-\end{array}$ & $\begin{array}{l}-- \\
-- \\
-- \\
-- \\
--\end{array}$ \\
\hline $\begin{array}{l}846 \\
847 \\
848 \\
849 \\
850\end{array}$ & $\begin{array}{r}-- \\
10 \\
54 \\
2 \\
10\end{array}$ & $\begin{array}{c}-- \\
04-24-89 \\
05-17-89 \\
05-17-89 \\
11-04-89\end{array}$ & $\begin{array}{l}-- \\
-- \\
-- \\
- \\
--\end{array}$ & $\begin{array}{l}0-9 \text { sand, some silt and gravel; } 9 \text { BR. } \\
0-15 \text { sand, clayey silt, some gravel, cobbles, \& boulders. } \\
0-65 \text { sand mixed w/clayey silt \& gravel. } \\
0-22 \text { sand mixed w/silt \& clay, some gravel. } \\
0-2 \text { sand; } 2-15 \text { till; } 15-20 \text { sand \& gravel, mixed w/silt \& clay at } 20 ; 20-30 \mathrm{SH} \text {. }\end{array}$ \\
\hline
\end{tabular}


Table 1.--Records of wells and test holes--continued.

[Dashes indicate no data. Locations shown on pls. 1A, 1B.]

\begin{tabular}{|c|c|c|c|c|c|c|c|c|c|c|}
\hline \multirow[b]{2}{*}{$\begin{array}{c}\text { Well } \\
\text { number }\end{array}$} & \multicolumn{3}{|c|}{ Location } & \multirow[b]{2}{*}{ Owner } & \multirow{2}{*}{\multicolumn{2}{|c|}{$\begin{array}{l}\text { Altitude } \\
\text { of land } \\
\text { surface } \\
\text { (feet) }\end{array}$}} & \multirow[b]{2}{*}{$\begin{array}{l}\text { Well } \\
\text { depth } \\
\text { (feet) }\end{array}$} & \multirow{2}{*}{\multicolumn{2}{|c|}{$\begin{array}{l}\text { Depth } \\
\text { to } \\
\text { bedrock } \\
\text { (feet) }\end{array}$}} & \multirow[b]{2}{*}{$\begin{array}{c}\text { Geologic } \\
\text { unit }\end{array}$} \\
\hline & $\begin{array}{l}\text { Lati- } \\
\text { tude } \\
\ldots n\end{array}$ & $\begin{array}{c}\text { Longi- } \\
\text { tude } \\
\ldots n\end{array}$ & $\begin{array}{l}\text { Accu- } \\
\text { racy }\end{array}$ & & & & & & & \\
\hline $\begin{array}{l}851 \\
852 \\
853 \\
854 \\
855\end{array}$ & $\begin{array}{l}423028 \\
423418 \\
424049 \\
422102 \\
423537\end{array}$ & $\begin{array}{l}0755050 \\
0754024 \\
0754252 \\
0754147 \\
0753219\end{array}$ & $\begin{array}{l}F \\
F \\
F \\
F \\
F\end{array}$ & $\begin{array}{l}\text { German Landfill } \\
\text { Pharsalia Landfill } \\
\text { Otselic Landfill } \\
\text { Dist. 1 Landfill } \\
\text { N. Norwich Landfill }\end{array}$ & $\begin{array}{l}11-02-89 \\
02-13-90 \\
06-01-83 \\
02-11-87 \\
01-09-84\end{array}$ & $\begin{array}{r}1559 \\
1810 \\
1593 \\
945 \\
1165\end{array}$ & $\begin{array}{l}55 \\
57 \\
46 \\
87 \\
45\end{array}$ & $\begin{array}{l}-- \\
-- \\
-- \\
-- \\
--\end{array}$ & $\begin{array}{c}49 \\
-- \\
-- \\
-- \\
28 .\end{array}$ & $\begin{array}{l}\text { BDRK } \\
\text { QRNR } \\
\text { QRNR } \\
\text { QRNR } \\
\text { BDRK }\end{array}$ \\
\hline $\begin{array}{l}856 \\
857 \\
858\end{array}$ & $\begin{array}{l}423525 \\
421946 \\
421943\end{array}$ & $\begin{array}{l}0753212 \\
0752443 \\
0752509\end{array}$ & $\begin{array}{l}F \\
F \\
F\end{array}$ & $\begin{array}{l}\text { N. Norwich Landfill } \\
\text { Bags San. Landfill } \\
\text { Bags San. Landfill }\end{array}$ & $\begin{array}{l}12-20-83 \\
11-16-87 \\
05-05-87\end{array}$ & $\begin{array}{l}1080 \\
1111 \\
1117\end{array}$ & $\begin{array}{r}60 \\
122 \\
80\end{array}$ & $\begin{array}{l}-- \\
-- \\
--\end{array}$ & $\begin{array}{l}-- \\
68\end{array}$ & $\begin{array}{l}\text { QRNR } \\
\text { QRNR } \\
\text { BDRK }\end{array}$ \\
\hline 859 & 423325 & 0753128 & $\mathbf{F}$ & Norwich Eaton Pharm. & $05-16-82$ & 1005 & 62 & -- & -- & QRNR \\
\hline 860 & 424039 & 0753024 & $\mathbf{F}$ & Gen. Instr. Corp. & $12-14-72$ & 1010 & 280 & -- & -- & QRNR \\
\hline 861 & 424045 & 0753042 & $\mathbf{F}$ & Gen. Instr. Corp. & $10-29-72$ & 1015 & 225 & - & 222 & BDRK \\
\hline $\begin{array}{l}862 \\
863 \\
864 \\
865\end{array}$ & $\begin{array}{l}424134 \\
421817 \\
422824 \\
422819\end{array}$ & $\begin{array}{l}0753009 \\
0752819 \\
0752743 \\
0752802\end{array}$ & $\begin{array}{l}F \\
F \\
F \\
F\end{array}$ & $\begin{array}{l}\text { Gen. Instr. Corp. } \\
\text { Bainbridge Resin } \\
\text { Norwich Landfill } \\
\text { Norwich Landfill }\end{array}$ & $\begin{array}{l}10-18-72 \\
02-26-81 \\
02-22-90 \\
02-27-90\end{array}$ & $\begin{array}{l}1050 \\
1004 \\
1623 \\
1682\end{array}$ & $\begin{array}{r}135 \\
42 \\
52 \\
40\end{array}$ & $\begin{array}{l}-- \\
-- \\
-- \\
--\end{array}$ & $\begin{array}{r}134 \\
-- \\
- \\
26\end{array}$ & $\begin{array}{l}\text { BDRK } \\
\text { QRNR } \\
\text { QRNR } \\
\text { BDRK }\end{array}$ \\
\hline $\begin{array}{l}866 \\
867 \\
868 \\
869\end{array}$ & $\begin{array}{l}423148 \\
423214 \\
423227 \\
423236\end{array}$ & $\begin{array}{l}0754435 \\
0753045 \\
0753058 \\
0753116\end{array}$ & $\begin{array}{l}F \\
S \\
S \\
F\end{array}$ & $\begin{array}{l}\text { Novak Farms } \\
\text { City of Norwich } \\
\text { Borden Milk Co. } \\
\text { City of Norwich }\end{array}$ & $\begin{array}{l}09-05-84 \\
10-13-65 \\
10-18-46 \\
10-01-75\end{array}$ & $\begin{array}{r}1520 \\
995 \\
1000 \\
995\end{array}$ & $\begin{array}{r}46 \\
27 \\
100 \\
233\end{array}$ & $\begin{array}{r}-- \\
27 \\
100 \\
204\end{array}$ & $\begin{array}{l}-- \\
- \\
-- \\
--\end{array}$ & $\begin{array}{l}\text { QRNR } \\
\text { QRNR } \\
\text { QRNR } \\
\text { QRNR }\end{array}$ \\
\hline 870 & 423559 & 0753116 & $\mathbf{S}$ & Norwich Eaton Pharm. & $08-29-75$ & 1010 & 303 & -- & 303 & BDRK \\
\hline 871 & 423558 & 0753128 & $\mathbf{S}$ & Norwich Eaton Pharm. & $06-01-75$ & 1010 & 335 & - & 325 & BDRK \\
\hline 872 & 423604 & 0753124 & $\mathbf{S}$ & Norwich Eaton Pharm. & $06-01-75$ & 1010 & 260 & -- & 231 & BDRK \\
\hline $\begin{array}{l}873 \\
874 \\
875\end{array}$ & $\begin{array}{l}423611 \\
423622 \\
421754\end{array}$ & $\begin{array}{l}0753149 \\
0753143 \\
0752545\end{array}$ & $\begin{array}{l}S \\
S \\
F\end{array}$ & $\begin{array}{l}\text { NYSDOT } \\
\text { NYSDOT } \\
\text { Chenango County }\end{array}$ & 11- $\overline{--}$ & $\begin{array}{r}1026 \\
1027 \\
975\end{array}$ & $\begin{array}{l}32 \\
42 \\
52\end{array}$ & $\begin{array}{l}-- \\
-- \\
-\end{array}$ & $\begin{array}{l}-- \\
--\end{array}$ & $\begin{array}{l}\text { QRNR } \\
\text { QRNR } \\
\text { QRNR }\end{array}$ \\
\hline $\begin{array}{l}876 \\
877 \\
878 \\
879 \\
880\end{array}$ & $\begin{array}{l}423555 \\
422657 \\
422650 \\
422502 \\
421742\end{array}$ & $\begin{array}{l}0753141 \\
0753518 \\
0753505 \\
0753728 \\
0752630\end{array}$ & $\begin{array}{l}F \\
S \\
S \\
F \\
F\end{array}$ & $\begin{array}{l}\text { Norwich Eaton Pharm. } \\
\text { NYSDOT } \\
\text { NYSDOT } \\
\text { Williams } \\
\text { Chenango County }\end{array}$ & $\begin{array}{l}01-01-75 \\
08-01-79 \\
12-01-75 \\
06-01-80 \\
11-06-68\end{array}$ & $\begin{array}{r}1010 \\
977 \\
960 \\
1075 \\
975\end{array}$ & $\begin{array}{r}364 \\
61 \\
125 \\
104 \\
46\end{array}$ & $\begin{array}{c}-- \\
61 \\
-- \\
22 \\
--\end{array}$ & $\begin{array}{r}364 \\
-- \\
-- \\
12 \\
44\end{array}$ & $\begin{array}{l}\text { QRNR } \\
\text { QRNR } \\
\text { QRNR } \\
\text { BDRK } \\
\text { BDRK }\end{array}$ \\
\hline $\begin{array}{l}881 \\
882 \\
883 \\
884 \\
885\end{array}$ & $\begin{array}{l}421757 \\
421751 \\
421733 \\
421714 \\
421633\end{array}$ & $\begin{array}{l}0752658 \\
0752754 \\
0752828 \\
0752851 \\
0752833\end{array}$ & $\begin{array}{l}\text { F } \\
\text { F } \\
\text { F } \\
\text { F } \\
\text { F }\end{array}$ & $\begin{array}{l}\text { Chenango County } \\
\text { Chenango County } \\
\text { Chenango County } \\
\text { Chenango County } \\
\text { Chenango County }\end{array}$ & $\begin{array}{l}11-06-68 \\
11-06-68 \\
11-05-68 \\
11-06-68 \\
11-07-68\end{array}$ & $\begin{array}{l}975 \\
975 \\
975 \\
975 \\
975\end{array}$ & $\begin{array}{l}52 \\
52 \\
52 \\
52 \\
52\end{array}$ & $\begin{array}{l}-- \\
-- \\
-- \\
--\end{array}$ & $\begin{array}{l}-- \\
-- \\
-- \\
--\end{array}$ & $\begin{array}{l}\text { QRNR } \\
\text { QRNR } \\
\text { QRNR } \\
\text { QRNR } \\
\text { QRNR }\end{array}$ \\
\hline $\begin{array}{l}886 \\
887 \\
888 \\
889 \\
890\end{array}$ & $\begin{array}{l}421548 \\
423440 \\
423530 \\
423620 \\
423711\end{array}$ & $\begin{array}{l}0752902 \\
0753120 \\
0753123 \\
0753117 \\
0753046\end{array}$ & $\begin{array}{l}\text { F } \\
\text { F } \\
\text { F } \\
\text { F } \\
\text { F }\end{array}$ & $\begin{array}{l}\text { Chenango County } \\
\text { Chenango County } \\
\text { Chenango County } \\
\text { Chenango County } \\
\text { Chenango County }\end{array}$ & $\begin{array}{l}11-07-68 \\
10-23-68 \\
10-24-68 \\
10-29-68 \\
10-30-68\end{array}$ & $\begin{array}{r}975 \\
1000 \\
1000 \\
1000 \\
1010\end{array}$ & $\begin{array}{l}52 \\
52 \\
52 \\
52 \\
91\end{array}$ & $\begin{array}{l}- \\
- \\
-- \\
--\end{array}$ & $\begin{array}{l}-- \\
-- \\
-- \\
-- \\
--\end{array}$ & $\begin{array}{l}\text { QRNR } \\
\text { QRNR } \\
\text { QRNR } \\
\text { QRNR } \\
\text { QRNR }\end{array}$ \\
\hline
\end{tabular}


Table 1.--Records of wells and test holes--continued.

[Dashes indicate no data. Locations shown on pls. 1A, 1B.]

\begin{tabular}{|c|c|c|c|c|}
\hline \multirow{2}{*}{$\begin{array}{c}\text { Well } \\
\text { number }\end{array}$} & \multicolumn{2}{|c|}{ Water level } & \multirow{2}{*}{$\begin{array}{l}\text { Yield } \\
\text { (gal/min) }\end{array}$} & \multirow{2}{*}{$\begin{array}{l}\text { Remarks } \\
\text { (Depths of geologic units given in feet below land surface) }\end{array}$} \\
\hline & feet & date & & \\
\hline 851 & 25 & $03-06-90$ & $\cdots$ & 0-5 clayey silt; $5-49$ silt \& clay (till?); $49-55$ STS \& SH. \\
\hline 852 & -- & -- & -- & 0-57 clayey silt, some sand \& gravel (till?). \\
\hline 853 & 10 & $06-06-83$ & -- & 0-28 sand; $28-36$ sand \& gravel; $36-40$ sand \& silt; $40-46$ sand. \\
\hline 854 & -- & -- & -- & $0-6$ sand; $6-37$ clayey silt; $37-51$ sand; $51-72$ gravel; $72-87$ sand. \\
\hline 855 & 28 & $01-09-84$ & -- & $0-28$ gravel, some sand \& silt (till?); 28 SH or STS. \\
\hline 856 & 28 & $12-20-83$ & -- & $0-60$ silt \& sand (till?); 60 SH chips. \\
\hline 857 & 114 & $02-03-88$ & -- & $0-8$ silt \& sand; $8-122$ sand \& gravel. \\
\hline 858 & 20 & $05-05-87$ & -- & $\begin{array}{l}0-25 \text { sand \& gravel; } 25-58 \text { silt; } 58-68 \text { sand \& gravel, weath. SH; } 68-80 \text { SH \& } \\
\text { STS. }\end{array}$ \\
\hline 859 & 21 & $05-16-82$ & -- & $\begin{array}{l}0-16 \text { sand; } 16-18 \text { sand \& gravel; } 18-48 \text { sand; } 48-54 \text { sand \& silt; } 54-58 \text { sand; } 58- \\
62 \text { silt. }\end{array}$ \\
\hline 860 & -- & -- & -- & $0-25$ sand \& fine gravel; $25-180$ clay; $180-185$ HP; $185-215$ clay; $215-280$ silt. \\
\hline 861 & -- & -- & -- & $\begin{array}{l}\text { 0-175 clay; } 175-190 \text { silty clay; } 190-214 \text { silt; } 214-215 \text { HP; } 215-222 \text { silty sand; } \\
222-225 \text { BR. }\end{array}$ \\
\hline 862 & -- & -- & -- & $0-10$ silty sand \& fine gravel; $10-115$ clay; $115-127$ sand \& gravel; $127-134$ sand. \\
\hline 863 & 3 & $03-06-81$ & -- & $0-42$ silty sand \& gravel. \\
\hline 864 & -- & -- & -- & $0-52$ till. \\
\hline 865 & -- & -- & -- & 0-26 till; $26-40 \mathrm{SS}$. \\
\hline 866 & 32 & $09-05-84$ & -- & $0-5$ silt; $5-10$ sand \& gravel; $10-20$ silt, gravel; $20-32$ sand \& silt; $32-46$ silt. \\
\hline 867 & 8 & $11-16-65$ & 170 & $0-4$ topsoil; $4-7$ clay; 7-27 flat \& round gravel w/sand. \\
\hline 868 & 12 & $11-05-46$ & 1000 & 0-2 fill, 2-5 clay \& gravel; 5-31 coarse sand \& gravel, boulders; $31-100$ clay. \\
\hline 869 & 8 & $11-04-75$ & 1018 & $\begin{array}{l}0-17 \text { clay w/sand \& gravel; } 17-186 \text { clay; } 186-228 \text { sand w/boulders; } 228-233 \\
\text { sand. }\end{array}$ \\
\hline 870 & 11 & $08-29-75$ & -- & $\begin{array}{l}0-40 \text { sand \& gravel; } 40-145 \text { silt; } 145-160 \text { sand; } 160-303 \text { silt, clay, sand \& gravel; } \\
303 \text { BR. }\end{array}$ \\
\hline 871 & -- & -- & -- & $\begin{array}{l}\text { 0-31 gravel; } 31-65 \text { clay, sandy w/gravel; } 65-193 \text { sand \& clay; } 193-325 \text { gravel; } \\
325 \text { BR. }\end{array}$ \\
\hline 872 & -- & $\cdots$ & -- & $\begin{array}{l}0-37 \text { gravel w/silt; } 37-182 \text { clay w/sand \& silt; } 182-231 \text { gravel w/cobbles; } 231 \\
\text { BR. }\end{array}$ \\
\hline 873 & -- & -- & -- & $0-5$ silt; $5-10$ gravel; $10-15$ silt; $15-20$ sandy gravel; $20-32$ sand \& gravel. \\
\hline 874 & -- & -- & -- & $0-5$ sandy gravel; $5-42$ clay \& silt. \\
\hline 875 & 15 & $11-04-68$ & -- & $0-11$ silt \& fine sand; $11-25$ sand \& gravel; $25-30$ sand $w /$ silt; $30-52$ silt $w /$ sand. \\
\hline 876 & -- & -- & -- & 0-18 gravel; 18-137 clay; $137-187$ gravel; $187-364$ gravel w/sand, silt \& clay. \\
\hline 877 & 15 & $08-15-79$ & -- & 0-10 gravel; $10-21$ sandy silt; $21-31$ silt; $31-41$ sandy silt; 41-61 sandy gravel. \\
\hline 878 & 7 & $12-01-75$ & -- & $0-103$ silty sand; $103-108$ clay; $108-125$ sandy gravel. \\
\hline 879 & 25 & $06-01-80$ & 10 & $0-12 \mathrm{HP}$ and boulders; $12-22$ soft $\mathrm{SH} ; 22-104 \mathrm{red} / \mathrm{gray} \mathrm{SH}$. \\
\hline 880 & 16 & $11-06-68$ & -- & $\begin{array}{l}0-30 \text { sand, some silt; } 30-35 \text { silt, some gravel; } 35-44 \text { gravel \& silt; } 44-46 \text { sandy } \\
\text { STS \& SH. }\end{array}$ \\
\hline 881 & 12 & $11-06-68$ & -- & $0-10$ silt \& clay; $10-20$ sand; $20-26$ sand \& gravel w/silt; $26-52$ silt w/sand. \\
\hline 882 & 17 & $11-06-68$ & -- & $0-10$ silt; $10-16$ silt \& clay; $16-25$ sand \& silt; $25-52$ silt, w/some sand. \\
\hline 883 & 2 & $11-05-68$ &.- & $0-10$ silt \& med. gravel, grades to med. sand; $10-52$ silt \& fine sand. \\
\hline 884 & 18 & $11-06-68$ & -- & 0-16 fine sand, some silt; $16-30$ sand \& gravel, little silt; $30-52$ silt w/fine sand. \\
\hline 885 & 24 & $11-07-68$ & -- & $0-15$ silt, some clay \& sand; $15-20$ silt \& clay, fine sand; $20-52$ silt \& clay. \\
\hline 886 & 21 & $11-07-68$ & - & 0-35 fine to med. sand w/some silt; $35-40$ med. gravel; $40-52$ silt w/some clay. \\
\hline 887 & 10 & $10-23-68$ & -- & $0-9$ silt; $9-14$ sand \& gravel; $14-35$ silt \& clay; $35-45$ sand \& gravel; $45-52$ silt. \\
\hline 888 & 6 & $10-24-68$ & - & $0-4$ silt, some clay; $4-15$ sand, fine gravel, some silt; $15-52$ silt w/some clay. \\
\hline 889 & 7 & $10-29-68$ & -- & $0-6$ silt; $6-15$ sand; $15-28$ gravel, coarse sand; $28-52$ silt, some clay \& sand. \\
\hline 890 & 10 & $10-30-68$ & -- & $0-10$ silt; $10-20$ sand \& gravel; $20-91$ sand, silt, some gravel \\
\hline
\end{tabular}

